\title{
3 DOF Spherical Pendulum Oscillations with a Uniform Slewing Pivot Center and a Small Angle Assumption
}

\author{
Alexander V. Perig, ${ }^{1}$ Alexander N. Stadnik, ${ }^{2}$ \\ Alexander I. Deriglazov, ${ }^{2}$ and Sergey V. Podlesny ${ }^{2}$ \\ ${ }^{1}$ Manufacturing Processes and Automation Engineering Department, Engineering Automation Faculty, \\ Donbass State Engineering Academy, Shkadinova 72, Donetsk Region, Kramatorsk 84313, Ukraine \\ ${ }^{2}$ Department of Technical Mechanics, Engineering Automation Faculty, Donbass State Engineering Academy, \\ Shkadinova 72, Donetsk Region, Kramatorsk 84313, Ukraine
}

Correspondence should be addressed to Alexander V. Perig; olexander.perig@gmail.com

Received 11 February 2014; Revised 7 May 2014; Accepted 21 May 2014; Published 4 August 2014

Academic Editor: Mickaël Lallart

Copyright (C) 2014 Alexander V. Perig et al. This is an open access article distributed under the Creative Commons Attribution License, which permits unrestricted use, distribution, and reproduction in any medium, provided the original work is properly cited.

The present paper addresses the derivation of a 3 DOF mathematical model of a spherical pendulum attached to a crane boom tip for uniform slewing motion of the crane. The governing nonlinear DAE-based system for crane boom uniform slewing has been proposed, numerically solved, and experimentally verified. The proposed nonlinear and linearized models have been derived with an introduction of Cartesian coordinates. The linearized model with small angle assumption has an analytical solution. The relative and absolute payload trajectories have been derived. The amplitudes of load oscillations, which depend on computed initial conditions, have been estimated. The dependence of natural frequencies on the transport inertia forces and gravity forces has been computed. The conservative system, which contains first time derivatives of coordinates without oscillation damping, has been derived. The dynamic analogy between crane boom-driven payload swaying motion and Foucault's pendulum motion has been grounded and outlined. For a small swaying angle, good agreement between theoretical and averaged experimental results was obtained.

\section{Introduction}

Payload swaying dynamics during crane boom slewing is within the objectives and scope of many academic and industrial research programs in the fields of mechanical, electrical, and control engineering, and theoretical mechanics. Mathematical descriptions of relative and absolute payload swaying motion during crane boom rotation require the introduction of design models for a spherical pendulum with a suspension point following a horizontal circular trajectory. Spherical pendula with moving pivot centers are the so-called "eternal" problems that have been posed by ancient builders and civil engineers.

Today payload swaying problems attract the great attention of such applied mathematicians and mechanical engineers as Abdel-Rahman et al. [1-3], Adamiec-Wójcik et al. [4], Al-mousa et al. [5], Allan and Townsend [6], Aston [7],
Betsch et al. [8], Blackburn et al. $[9,10]$, Blajer et al. [1117], Cha et al. [18], Chin et al. [19], Ellermann et al. [20], Erneux and Kalmár-Nagy [21, 22], Ghigliazza and Holmes [23], Glossiotis and Antoniadis [24], Grigorov and Mitrev [25], Gusev and Vinogradov [26], Hong and Ngo [27], Hoon et al. [28], Ibrahim [29], Jerman et al. [30-33], Ju et al. [34], Kłosiński [35], Krukowski et al. [36, 37], Lenci et al. [38], Leung and Kuang [39], Loveykin et al. [40], Maleki et al. [41], Maczynski et al. [42-44], Marinović et al. [45], Masoud et al. [46, 47], Mijailović [48], Mitrev and Grigorov [49, 50], Morales et al. [51], Nakazono et al. [52], Neitzel et al. [53], Neupert et al. [54], O'Connor and Habibi [55], Omar and Nayfeh [56], Osiński and Wojciech [57], F. Palis and S. Palis [58], Perig et al. [59], Posiadała et al. [60], Ren et al. [61], Safarzadeh et al. [62], Sakawa et al. [63], Sawodny et al. [64], Spathopoulos et al. [65], Schaub [66], Solarz and Tora [67], Uchiyama et al. [68], Urbaś [69], and others. 
Applied engineering problems in the field of lifting-andtransport machines mainly deal with rectilinear or rotational motion of the spherical pendulum suspension center in determined and stochastic cases. Further improvement of rotating crane performance and efficiency requires the development of mathematical models for an adequate description of payload and crane boom tip positions. Modern computational approaches to the solution of payload positioning problems have been investigated in the following works.

Abdel-Rahman et al. [1-3] have provided a comprehensive review of different types of cranes, the essential and widely used mathematical techniques for models of crane dynamics and classical control methods [1-3]. However, this research [1-3] gives inadequate attention to the effects of Coriolis inertia forces on the payload relative motion during crane boom slew motion.

Blajer et al. [11-17] have proposed thirteen index-three differential-algebraic equations (DAE) in the rotary crane state variables and control variables [11-17]. They have proposed governing DAE equations and stable solution techniques allowing the rotary crane to execute the prescribed load trajectory and the control commands to implement feed-forward control. In this approach [11-17] the governing equations have been derived without consideration of the Coriolis inertia force and non-zero horizontal projections of the load gravity force.

Hairer et al. [70, 71] and Øksendal [72] have developed computational techniques for DAE systems numerical integration.

Jerman et al. [30-33] have applied an enhanced mathematical model of slewing the crane motion with load pendulation, taking into account system stiffness coefficients [3033]. All mathematical techniques and governing equations in these works [30-33] have been presented in implicit form.

$\mathrm{Ju}$ et al. have implemented finite element simulation for a flexible crane structure with a spherical pendulum [34]. In this work [34], the spherical pendulum excitation is induced by vibration modes of the flexible crane structure.

Loveykin et al. have derived the law of an optimal control for lifting-and-shifting machines under the assumption of minimization of quadratic performance criteria in the case for two-phase coordinates, control and control rate [40]. This work [40] made wide use of variation optimization techniques for pendulum oscillations in the vertical plane, which involve the trolleys of crane frames with rectilinear motion.

Maczynski et al. have applied a numerically based finite element method (FEM) approach for simulation of a "crane boom-payload" system without an explicit introduction of inertia forces [42-44]. Optimization problem of load positioning in this study [42-44] has not fully addressed the natural frequencies estimation for the system "crane boompayload" in the case of a fully rigid crane boom model.

Mitrev and Grigorov [49, 50] have derived governing equations for load relative swaying taking into account energy dissipation, centrifugal, Coriolis, inertia, and gravity forces $[49,50]$. The Lagrange equations used here allow the simulation of a spherical pendulum with a movable pivot center $[49,50]$. Mitrev's approach $[49,50]$ is based on the introduction of angular generalized coordinates which result in nonlinearity of the problems and require a fourth-order Runge-Kutta fixed-step integration method.

However the previously known studies [1-100] have given inadequate attention to the dynamic analysis of a load swaying in the horizontal plane of vibrations while accounting for the effect of the Coriolis force on the trajectory of the relative load motion of the cable. The present research addresses this situation.

It should be noted that spherical pendulum related research has also been further developed for Foucault pendulums in the works of Condurache and Martinusi [77], Gusev and Vinogradov [26], de Icaza-Herrera and Castoño [83], Pardy [88], Zanzottera et al. [99], Zhuravlev and Petrov [100], and others.

At first sight, the spherical pendulum with rotating pivot center and Foucault pendulum are two vastly different dynamic systems. The key difference between the two dynamic systems is that dynamic analysis of the Foucault pendulum is focused on a load swaying in the field of the central gravity force, while crane boom slewing problems are posed for the vertical gravity force. A commonality of the two dynamic systems is, in both cases, the effect of influence of normal centrifugal and Coriolis forces on the shape of the relative and absolute trajectories. The normal centrifugal forces depend on the relative coordinates and Coriolis forces depend on the relative velocity of the swaying load. Moreover, the appearance of Coriolis forces, that are dependent on relative payload velocity, retains the dynamic system as a conservative one because Coriolis acceleration remains at all times perpendicular to the relative velocity of the load. It may therefore be concluded that there is a close coupling between the spherical pendulum with rotating pivot center and Foucault pendulum. Moreover the close relationship between the two dynamic systems has not been properly addressed in all previous known research, which emphasizes the actuality and relevance of the present paper.

The present paper is focused on the study of the oscillation processes taking place in the vicinity of a steady equilibrium position of a payload during crane boom uniform slewing. The computational approach is based on the solution of the initial value problem of particle dynamics for the determination of the relative load trajectory in the horizontal plane of the vibrations during crane boom uniform rotation. The approach used here takes into account both the relative rotation of the load vibrations in the vertical plane and the influence of Coriolis acceleration on the form of the trajectory of the swaying cargo relative motion. This paper is also aimed at addressing the physically grounded interrelations between the spherical pendulum with rotating pivot center and Foucault pendulum.

\section{DAE System for Payload Swaying}

It has been shown in Appendices $\mathrm{A}-\mathrm{O}$ that the nonlinear DAE system (formulae (C.1), (F.7)) for the relative coordinates $x_{1}(t)$ (Figure $\left.1(\mathrm{a})\right) ; y_{1}(t)$ (Figure $\left.1(\mathrm{~b})\right) ; z_{1}(t)$ of a swaying 


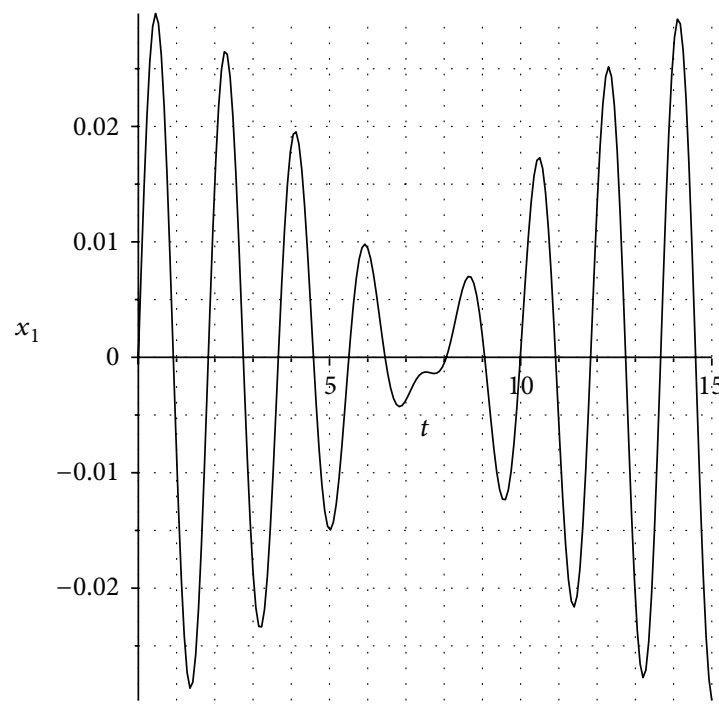

(a)

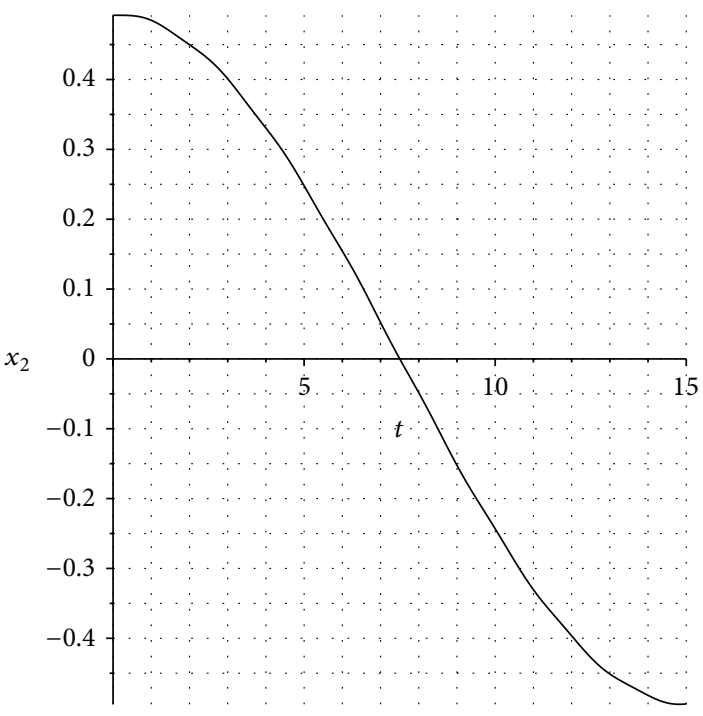

(c)

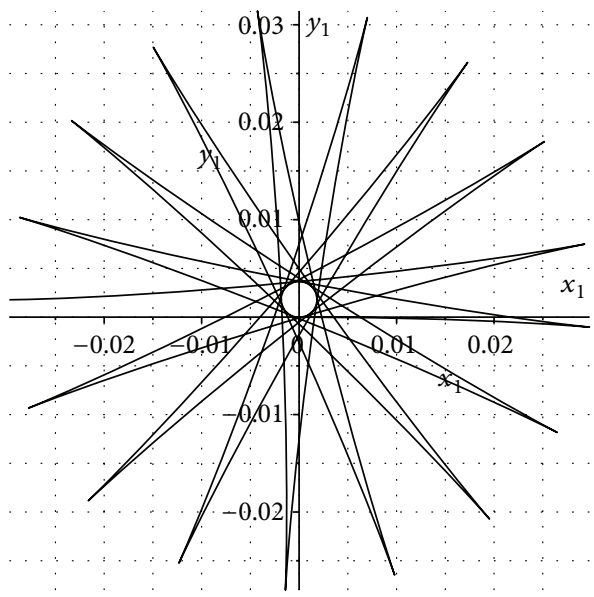

(e)

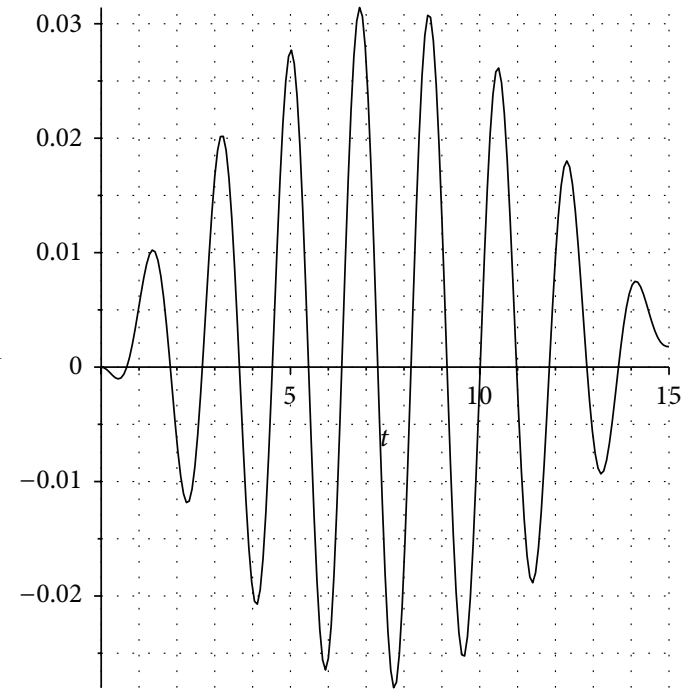

(b)

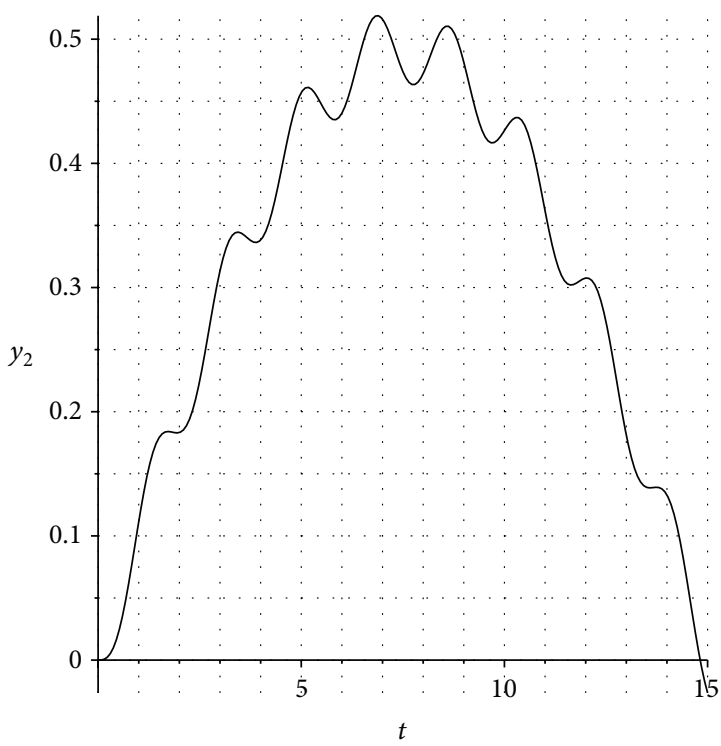

(d)

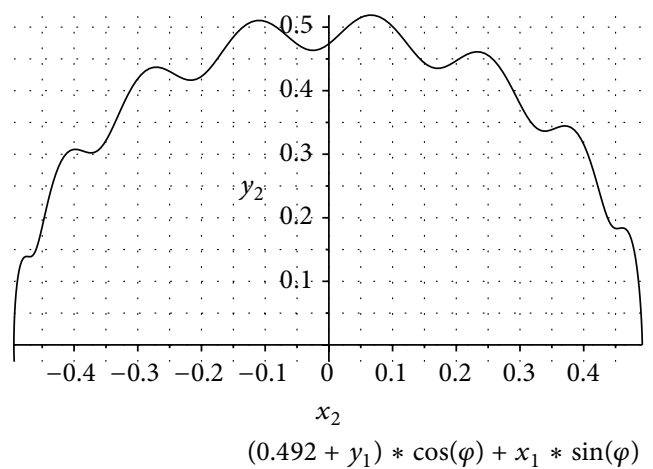

(f)

FIGURE 1: Computational relative (a-b, e) and computational absolute (c-d, f) coordinates of a swaying payload during uniform crane boom slewing for the following numerical values of system parameters: $m=0.1 \mathrm{~kg} ; l=0.825 \mathrm{~m} ; R=0.492 \mathrm{~m} ; g=9.81 \mathrm{~m} / \mathrm{s}^{2} ;$ and $\omega_{e}=0.209 \mathrm{rad} / \mathrm{s}$. 
payload during crane boom slewing (Figures 8-10) in the noninertial reference frame $\mathscr{B}$ is as follows:

$$
\begin{aligned}
& m\left(\frac{d^{2}\left(x_{1}(t)\right)}{d t^{2}}\right) \\
& =-N(t)\left(\frac{x_{1}(t)}{l}\right)+m\left(\frac{d\left(\varphi_{e}(t)\right)}{d t}\right)^{2} x_{1}(t) \\
& +m\left(\frac{d^{2}\left(\varphi_{e}(t)\right)}{d t^{2}}\right)\left(R+y_{1}(t)\right) \\
& +2 m\left(\frac{d\left(\varphi_{e}(t)\right)}{d t}\right)\left(\frac{d\left(y_{1}(t)\right)}{d t}\right) ; \\
& m\left(\frac{d^{2}\left(y_{1}(t)\right)}{d t^{2}}\right) \\
& =-N(t)\left(\frac{y_{1}(t)}{l}\right)+m\left(\frac{d\left(\varphi_{e}(t)\right)}{d t}\right)^{2}\left(R+y_{1}(t)\right) \\
& -m\left(\frac{d^{2}\left(\varphi_{e}(t)\right)}{d t^{2}}\right) x_{1}(t) \\
& -2 m\left(\frac{d\left(\varphi_{e}(t)\right)}{d t}\right)\left(\frac{d\left(x_{1}(t)\right)}{d t}\right) ; \\
& m\left(\frac{d^{2}\left(z_{1}(t)\right)}{d t^{2}}\right)=-m g+N(t)\left(\frac{l-z_{1}(t)}{l}\right) ; \\
& l^{2}=\left(x_{1}(t)\right)^{2}+\left(y_{1}(t)\right)^{2}+\left(z_{1}(t)-l\right)^{2} \\
& \varphi_{e}(t)=\omega_{e} t ; \quad \varphi_{e}(0)=0 ; \\
& \frac{d\left(\varphi_{e}(0)\right)}{d t}=\omega_{e} ; \quad N(0)=m g ; \\
& x_{1}(0)=0 ; \quad \frac{d\left(x_{1}(0)\right)}{d t}=\omega_{e} R ; \quad y_{1}(0)=0 ; \\
& \frac{d\left(y_{1}(0)\right)}{d t}=0 ; \quad z_{1}(0)=0 ; \quad \frac{d\left(z_{1}(0)\right)}{d t}=0 .
\end{aligned}
$$

The absolute coordinates $x_{2}(t)$ (Figure $1(\mathrm{c})$ ); $y_{2}(t)$ (Figure $1(\mathrm{~d})$ ); $z_{2}(t)$ of payload $M$ in the inertial reference frame $\mathscr{E}$ may be computed as

$$
\begin{aligned}
& x_{2}(t)=\left(R+y_{1}(t)\right) \cos \left(\varphi_{e}(t)\right)+x_{1}(t) \sin \left(\varphi_{e}(t)\right) ; \\
& y_{2}(t)=\left(R+y_{1}(t)\right) \sin \left(\varphi_{e}(t)\right)-x_{1}(t) \cos \left(\varphi_{e}(t)\right) ; \\
& z_{2}(t)=z(t) .
\end{aligned}
$$

The absolute coordinates $x_{2}, y_{2}$ in the inertial reference frame $\mathscr{E}$, which depend upon the relative coordinates $x_{1}, y_{1}$ in the noninertial reference frame $\mathscr{B}$ during the swaying of load $M$ (Figures 8-10), has been defined according to the above mentioned Equations and has been shown in Figures 4 and 5 as $\left(---, y_{2}=y_{2}\left(x_{2}\right)\right)$.
The computational results in Figure 1, derived for DAE problem (1)-(2) solution, coincide with the linearized solution of the payload swaying problem in Appendices A-I (formula (H.1) in the noninertial reference frame $\mathscr{B}$ ).

It is necessary to note that the posed DAE problem (1) describes payload motion in the vicinity of the lower position of stable dynamical equilibrium assuming rope tension force $N(t)>0$. Factually, the applied rope is assumed as a unilateral geometric constraint (Appendix $\mathrm{C}$ ) in the shape of a torsion fiber. The upper position of the mechanical system is unstable (see $[73-75,78-80,91,93])$ and corresponds to the case of $N(t)<0$ within the parameters of the chosen mechanical system in Figures 8-10. Such an upper pendulum position might conflict with the hypothesis about the unilateral nature of geometric constraint. All of the above mentioned upper pendulum position conditions are outside the objectives and scope of the present paper.

\section{Experimental Procedure}

The experimental procedure has been grounded on the usage of the assembly in Figures 2 and 3.

The assembly in Figures 2 and 3 includes the following machine components: the vertical fixed shaft $\mathrm{O}_{2} \mathrm{D}$ with height $H=1 \mathrm{~m}$, the crane boom model $B D$ with length $R=0.5 \mathrm{~m}$ and diameter $6 \mathrm{~mm}$, the cable $B M$ with different fixed lengths $l_{1}=0.825 \mathrm{~m}$ (in Figure 2 and Figure $5(\mathrm{~b})$ ),$l_{2}=0.618 \mathrm{~m}$ (in Figure $5(\mathrm{a})$ ), $l_{3}=0.412 \mathrm{~m}$ (in Figure $4(\mathrm{~b})$ ), and $l_{4}=0.206 \mathrm{~m}$ (in Figure $4(\mathrm{a})$ ). The crane boom model $B D$ is attached to the vertical fixed shaft $\mathrm{O}_{2} D$ by bearing $D$. The cable $B M$ is attached to the crane boom $B D$ tip in the point $B$. The free or running end $M$ of the cable $B M$ is the payload $M$ attachment point. The load $M$ is a light emitting diode (LED) with diameter $2 \mathrm{~mm}$ and the battery with the battery voltage $3 \mathrm{~V}$. The experimental swaying trajectory $\left(-, y_{2}=y_{2}\left(x_{2}\right)\right)$ in Figures 2, 4, and 5 is the experimental light emitting diode $M$ absolute trajectory in the inertial reference frame $\mathscr{E}$.

The laser pointer in Figure 2 is also attached to the crane boom $B D$ tip in the point $B$. The laser pointer is the part of the noninertial reference frames $\mathscr{B}$ and $\mathscr{F}$ and the pointer trajectory is marked as (- - -) in Figure 2. The introduction of laser pointer $B$ allows estimation of dynamic deviation of payload $M$. The horizontal regular fixed grid with canvas size $1200 \mathrm{~mm} \times 700 \mathrm{~mm}$ is placed in the horizontal plane $\mathrm{O}_{2} x_{2} y_{2}$. The horizontal grid is formed by the square cells with size $20 \mathrm{~mm} \times 20 \mathrm{~mm}$.

The experimental swaying trajectories $\left(-, y_{2}=y_{2}\left(x_{2}\right)\right)$ in Figures 2, 4, and 5 have been written in the obscure room with the introduction of an upper digital camera with a long exposure $60 \mathrm{~s}-90 \mathrm{~s}$ and the camera height above horizontal grid level is $2.5 \mathrm{~m}$.

\section{Comparison and Discussion of DAE-Solution Based Theoretical and Experimental Results}

The comparison of DAE-solution based theoretical $\left(---, y_{2}=\right.$ $\left.y_{2}\left(x_{2}\right)\right)$ and experimental $\left(-, y_{2}=y_{2}\left(x_{2}\right)\right)$ results is shown in Figures 4 and 5. 


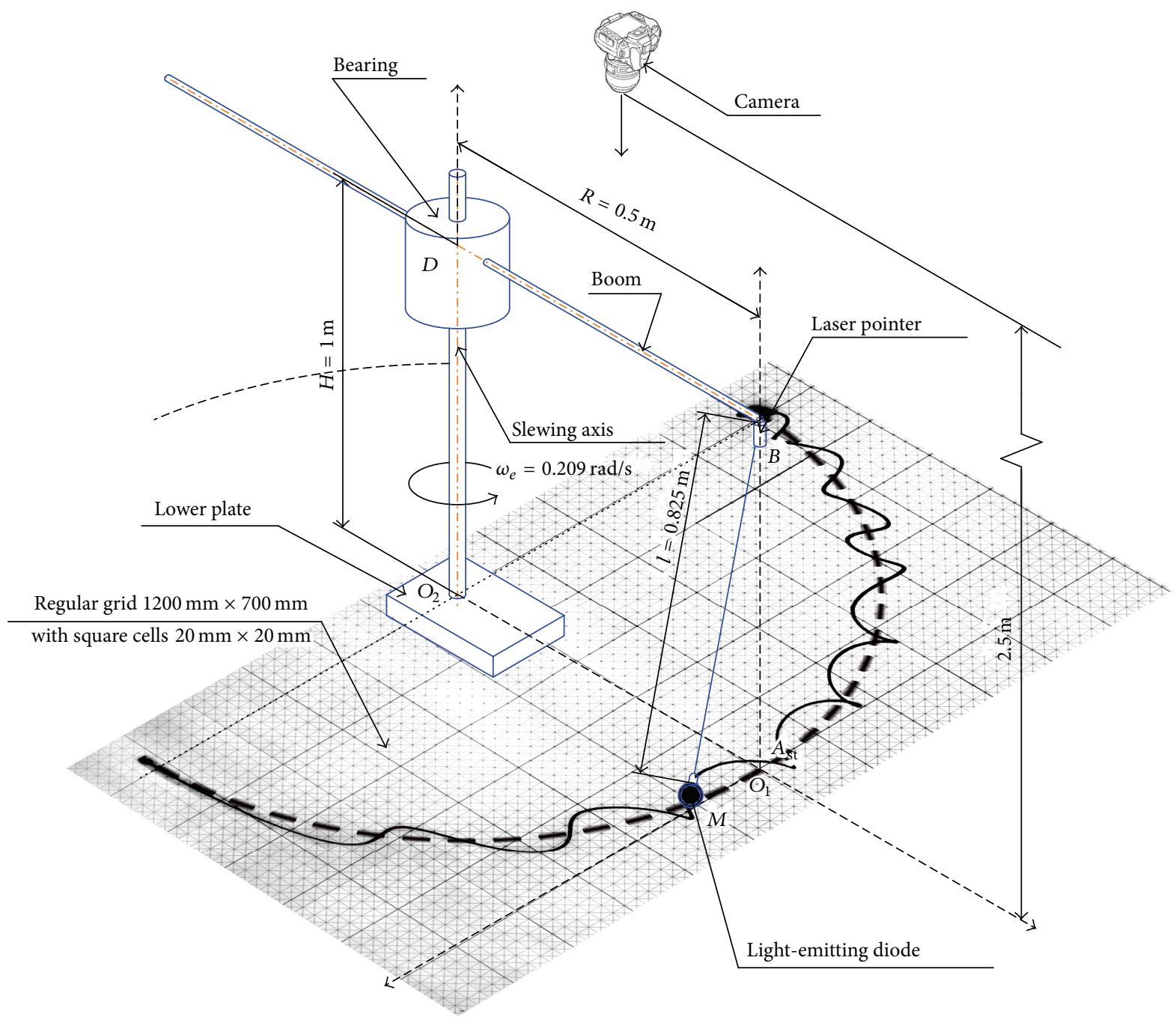

FIGURE 2: The dimension experimental measurement system for payload $M$ swaying motion during crane boom uniform slewing.

In order to estimate the relative disagreement of the derived DAE-solution based computational (- - -) and experimental (-) payload $M$ absolute trajectories in the inertial reference frame $\mathscr{E}$ we have computed the amplitude discrepancy $\delta$ in the polar coordinate system by the following formula:

$$
\delta=\left\langle\frac{1}{2}\left(\left(\frac{\left|r_{\text {comp }}-r_{\text {exper }}\right|}{r_{\text {comp }}}\right)+\left(\frac{\left|r_{\text {comp }}-r_{\text {exper }}\right|}{r_{\text {exper }}}\right)\right)\right\rangle,
$$

where $r_{\text {comp }}$ and $r_{\text {exper }}$ are the magnitudes of the radiusvectors, connecting point $\mathrm{O}_{2}$ and theoretical (- - ) and experimental (-) curves, and computed for the same fixed polar angle $\varphi_{e}$.

The amplitude discrepancies $\delta$ have the following values: $\delta=7.53 \%$ for $l=0.206 \mathrm{~m}$ in Figure $4(\mathrm{a}) ; \delta=7.58 \%$ for $l=0.412 \mathrm{~m}$ in Figure 4(b); $\delta=7.40 \%$ for $l=0.618 \mathrm{~m}$ in Figure 5(a); and $\delta=6.9 \%$ for $l=0.825 \mathrm{~m}$ in Figure 5(b).

Numerical computations (- -, $\left.y_{2}=y_{2}\left(x_{2}\right)\right)$ in Figure 4(a) have been carried out for the following values of mechanical system parameters: $R=0.492 \mathrm{~m} ; g=9.81 \mathrm{~m} / \mathrm{s}^{2}$; $l=0.206 \mathrm{~m} ; k=(\mathrm{g} / \mathrm{l})^{0.5} \approx 6.901 \mathrm{rad} / \mathrm{s} ; T=14 \mathrm{~s} ; \omega_{e}=2 \pi / T \approx$ $0.449 \mathrm{rad} / \mathrm{s} ; \varphi_{e}=180^{\circ} ; \alpha_{1 \mathrm{dvn}}=0.01014 \mathrm{rad} ; V_{B}=0.221 \mathrm{~m} / \mathrm{s}$; $y_{\mathrm{dyn}}=0.00209 \mathrm{~m} ; v_{1}=k+\omega_{e}=7.3496 \mathrm{rad} / \mathrm{s} ; v_{2}=k-$ $\omega_{e}=6.4520 \mathrm{rad} / \mathrm{s} ; C_{1}=0.01502 \mathrm{~m} ;$ and $C_{3}=0.01711 \mathrm{~m}$ (Figure 4(a)).

Numerical computations (- - , $\left.y_{2}=y_{2}\left(x_{2}\right)\right)$ in Figure 4(b) have been carried out for the following values of mechanical system parameters: $R=0.492 \mathrm{~m} ; g=9.81 \mathrm{~m} / \mathrm{s}^{2}$; $l=0.412 \mathrm{~m} ; k=(\mathrm{g} / \mathrm{l})^{0.5} \approx 4.879 \mathrm{rad} / \mathrm{s} ; T=14 \mathrm{~s} ; \omega_{e}=2 \pi / T \approx$ $0.449 \mathrm{rad} / \mathrm{s} ; \varphi_{e}=180^{\circ} ; \alpha_{1 \mathrm{dyn}}=0.01019 \mathrm{rad} ; V_{B}=0.221 \mathrm{~m} / \mathrm{s}$; $y_{\text {dyn }}=0.00419 \mathrm{~m} ; v_{1}=k+\omega_{e}=5.3284 \mathrm{rad} / \mathrm{s} ; v_{2}=k-$ $\omega_{e}=4.4308 \mathrm{rad} / \mathrm{s} ; C_{1}=0.02072 \mathrm{~m} ;$ and $C_{3}=0.02492 \mathrm{~m}$ (Figure 4(b)).

Numerical computations (- - - , $\left.y_{2}=y_{2}\left(x_{2}\right)\right)$ in Figure 5(a) have been carried out for the following values of mechanical system parameters: $R=0.492 \mathrm{~m} ; g=9.81 \mathrm{~m} / \mathrm{s}^{2} ; l=$ $0.618 \mathrm{~m} ; k=(\mathrm{g} / \mathrm{l})^{0.5} \approx 3.984 \mathrm{rad} / \mathrm{s} ; T=20 \mathrm{~s} ; \omega_{e}=2 \pi / T \approx$ $0.314 \mathrm{rad} / \mathrm{s} ; \varphi_{e}=180^{\circ} ; \alpha_{1 \mathrm{dyn}}=0.00498 \mathrm{rad} ; V_{B}=$ $0.1546 \mathrm{~m} / \mathrm{s} ; y_{\mathrm{dyn}}=0.00308 \mathrm{~m} ; v_{1}=k+\omega_{e}=4.298 \mathrm{rad} / \mathrm{s}$; 


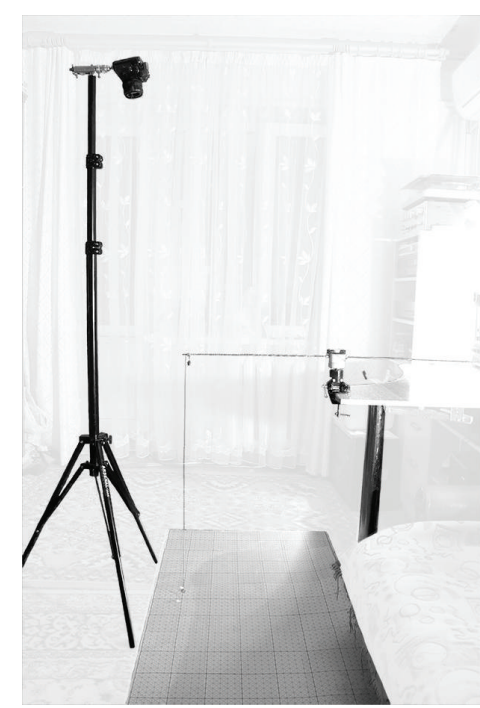

(a)

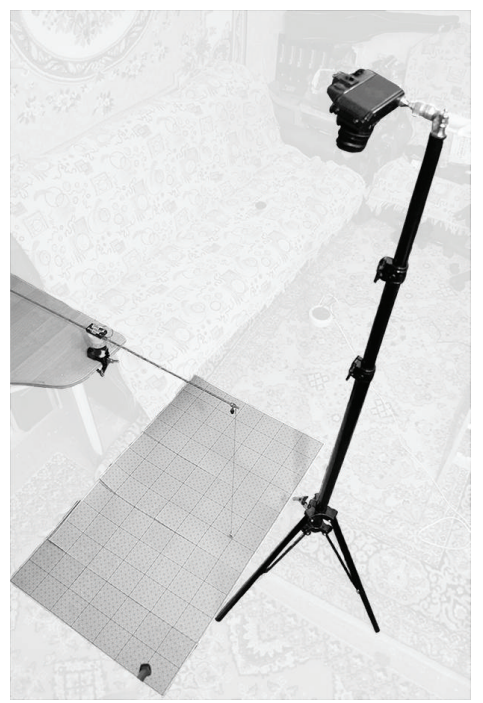

(c)

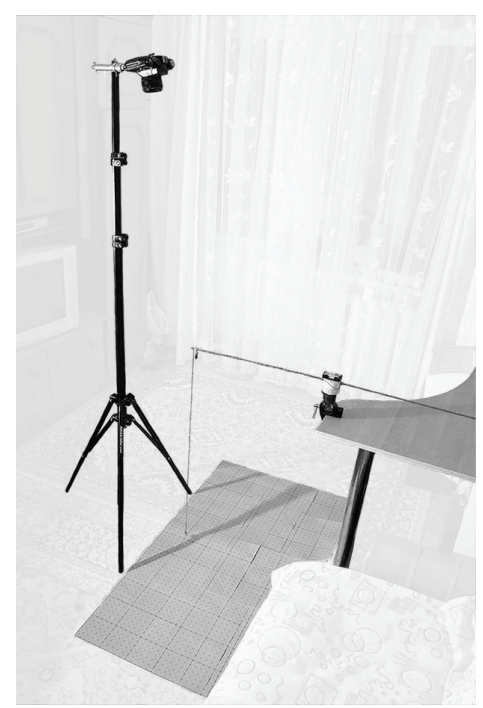

(b)

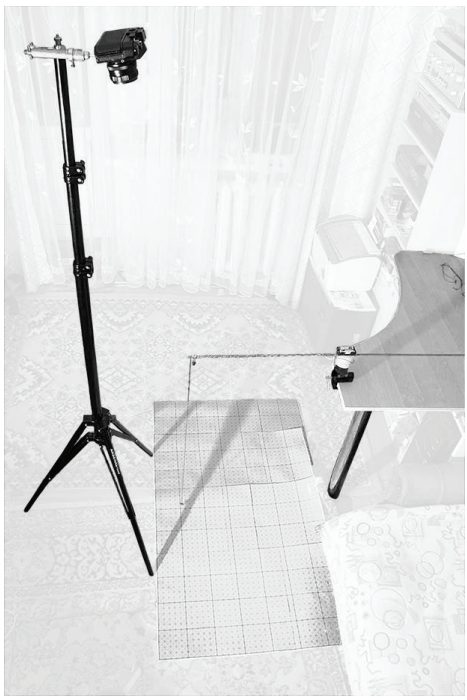

(d)

FIGURE 3: Photos of dimension experimental measurement system (a)-(d) for payload $M$ swaying motion during crane boom uniform slewing.

$\nu_{2}=k-\omega_{e}=3.670 \mathrm{rad} / \mathrm{s} ; C_{1}=0.01798 \mathrm{~m}$; and $C_{3}=$ $0.02106 \mathrm{~m}$ (Figure 5(a)).

For further estimation of the relative disagreement of derived DAE-solution based computational (- - -) and experimental (-) payload $M$ absolute trajectories in the inertial reference frame $\mathscr{E}$ we have computed the confidence intervals in Figures 4 and 5 for the dimensionless parameters $r_{\text {comp }} / r_{\text {exper }}$ and confidence probability 0.95 . The Student's $t$ test results yield the following confidence intervals: $0.9374 \leq$ $\left(r_{\text {comp }} / r_{\text {exper }}\right) \leq 1.0315$ for $l=0.206 \mathrm{~m}$ in Figure 4(a); $0.9014 \leq\left(r_{\text {comp }} / r_{\text {exper }}\right) \leq 1.0272$ for $l=0.412 \mathrm{~m}$ in Figure $4(\mathrm{~b}) ; 0.9200 \leq\left(r_{\text {comp }} / r_{\text {exper }}\right) \leq 1.0352$ for $l=0.618 \mathrm{~m}$ in Figure $5(\mathrm{a})$; and $0.9257 \leq\left(r_{\text {comp }} / r_{\text {exper }}\right) \leq 1.0066$ for $l=0.825 \mathrm{~m}$ in Figure 5(b).

Both the relative discrepancy $\delta$ and the confidence intervals show the satisfactory agreement between the absolute payload trajectories (Figures 4 and 5) in the inertial reference frame $\mathscr{E}$, that have been computed with DAE-solution based theoretical model (- - -) (1) and measured experimentally (-) as shown in Figures 4 and 5.

It is important to note that the payload motion DAE equations in the nonlinear problem (1) for the noninertial reference frame $\mathscr{B}$ may be derived with an introduction of Lagrange equations (Appendices A-C, F). However the discussion of (1) is more suitable and informative with an introduction of dynamic Coriolis theorem (Appendices A$\mathrm{D}, \mathrm{F})$. The first terms $d^{2} x_{1} / d t^{2} ; d^{2} y_{1} / d t^{2}$ in (1) define the vector $\mathbf{a}_{M / \mathscr{B}}=\mathbf{a}_{\mathbf{r}}$ (Appendix B, formula (B.4)) for the relative acceleration of load $M$ in the noninertial reference frame $\mathscr{B}$. The straight-line terms (D.3) formula-based terms in (1) are linearly proportional to the relative payload coordinates in the noninertial reference frame $\mathscr{B}$. These straight-line 


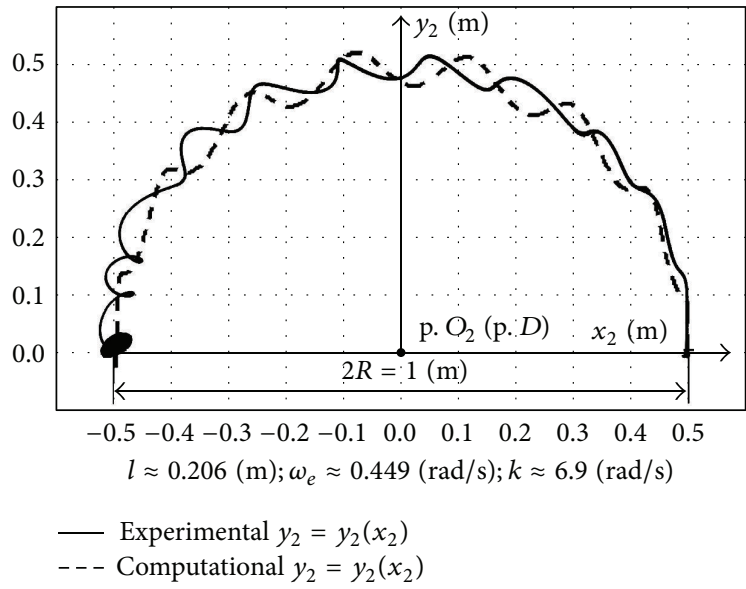

(a)

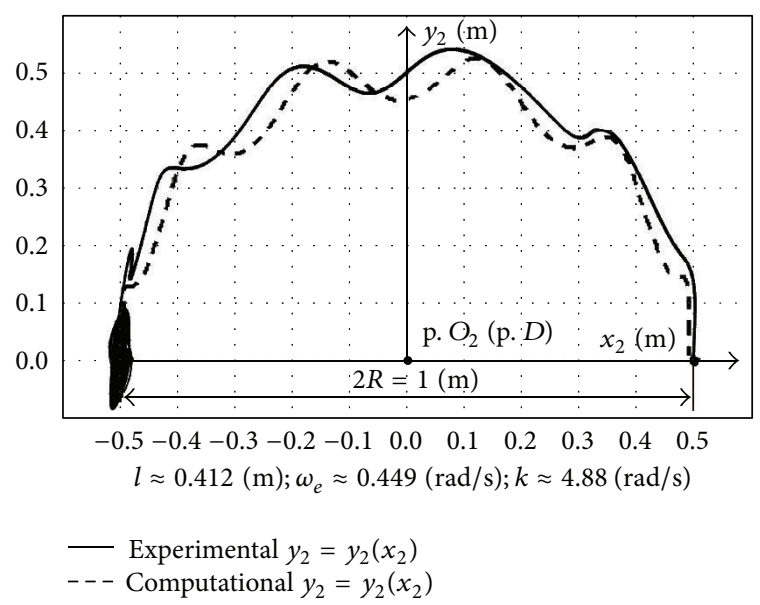

(b)

FIGURE 4: The comparison of the small swaying angle $\alpha_{1}$ theoretical $\left(---, y_{2}=y_{2}\left(x_{2}\right)\right)$ and experimental $\left(-, y_{2}=y_{2}\left(x_{2}\right)\right)$ load absolute trajectories in the inertial reference frame $\mathscr{E}$ for fixed cable length $l=0.206 \mathrm{~m}$ (a) and fixed cable length $l=0.412 \mathrm{~m}$ (b).

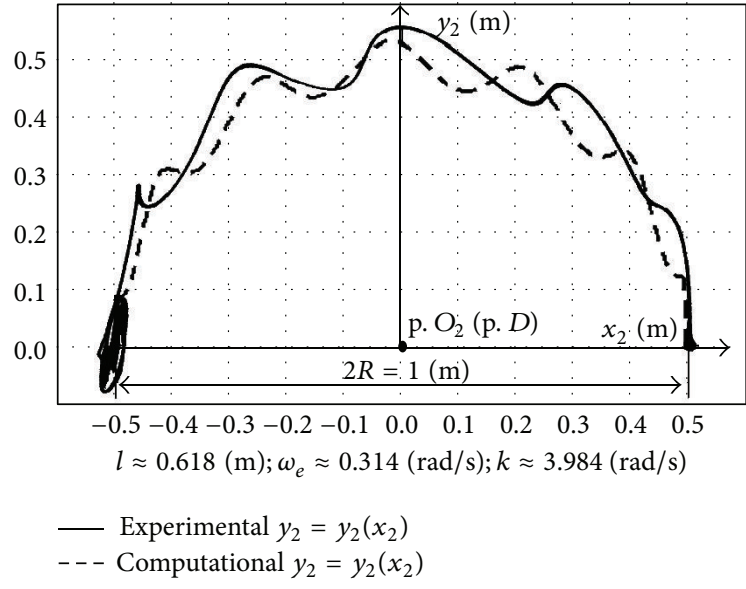

(a)

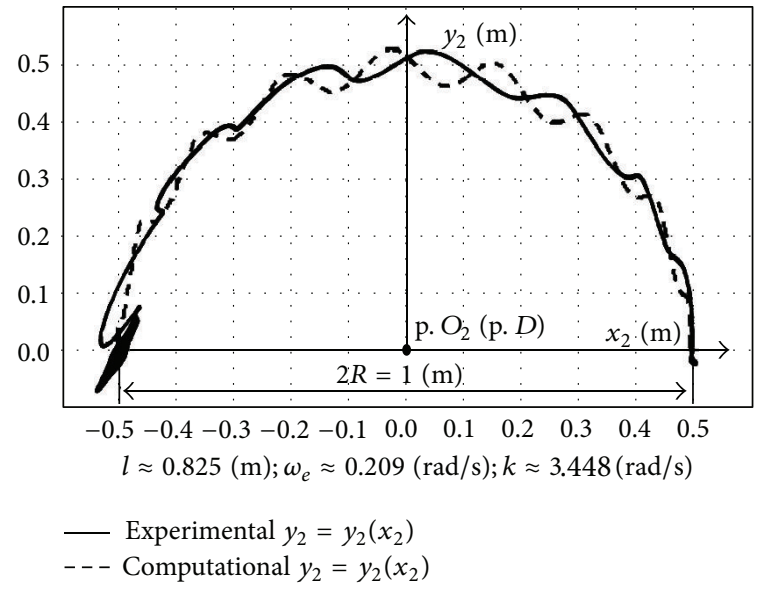

(b)

Figure 5: The comparison of the small swaying angle $\alpha_{1}$ theoretical $\left(--, y_{2}=y_{2}\left(x_{2}\right)\right)$ and experimental $\left(-, y_{2}=y_{2}\left(x_{2}\right)\right)$ load absolute trajectories in the inertial reference frame $\mathscr{E}$ for fixed cable length $l=0.618 \mathrm{~m}$ (a) and fixed cable length $l=0.825 \mathrm{~m}$ (b).

terms in (1) have been determined by the contribution of the normal or centripetal acceleration $\boldsymbol{\omega}_{\mathscr{B} / \mathscr{E}} \times\left(\boldsymbol{\omega}_{\mathscr{B} / \mathscr{E}} \times\right.$ $\left.\mathbf{r}_{E / M}\right)=\mathbf{a}_{\mathbf{e}}{ }^{\mathbf{n}}$ (B.6) of transportation for payload $M$ and by the appearance of corresponding D'Alembert centrifugal inertia force $\boldsymbol{\Phi}_{\mathbf{e}}{ }^{\mathbf{n}}=(-m) \mathbf{a}_{\mathbf{e}}{ }^{\mathbf{n}}$ (D.3) due to crane boom transport rotation in the noninertial reference frame $\mathscr{B}$. Additional terms $-2 \omega_{e}\left(d y_{1} / d t\right)$ and $2 \omega_{e}\left(d x_{1} / d t\right)$ in (1) have been defined by the compound or Coriolis acceleration $2 \boldsymbol{\omega}_{\mathscr{B} / \mathscr{E}} \times \mathbf{V}_{M / \mathscr{B}}=$ $\mathbf{a}_{\text {cor }}$ of payload $M$ in the noninertial reference frame $\mathscr{B}$. The rectangular Cartesian projections of Coriolis inertia force in the noninertial reference frame $\mathscr{B}$ is defined by formula (B.7).

\section{Experimental Results for the Spherical Pendulum with the Fixed Pivot Center}

Experimental load swaying results for the fixed crane boom model $\left(\omega_{e}=0 \mathrm{rad} / \mathrm{s}\right)$ are shown in Figure 6.
Finite motions of a swaying load are shown in Figure 6 in the case of $\omega_{e}=0 \mathrm{rad} / \mathrm{s}$. In Figure 6 the relative and absolute load trajectories are identically equal. The lingering remains of elliptical motion in Figure 6 and ellipses semiaxes are defined by the initial conditions.

\section{Discussion of Analogy between Payload Swaying and Foucault's Pendulum Motion}

The motion of Foucault's pendulum is shown in Figure 7, where $x_{2} y_{2} z_{2}$ is the heliocentric inertial reference frame (not shown in Figure 7), $x_{1} y_{1} z_{1}$ is the geocentric noninertial reference frame, and $x y z$ is a noninertial reference frame, located at the geographic latitude $y_{1} z ; A_{\mathrm{st}} z$ is a local vertical; $B D$ is the radius of the circle of the pinning point; $B M$ is the cable length; and $\omega_{e}$ is the angular velocity of the Earth diurnal rotation. 


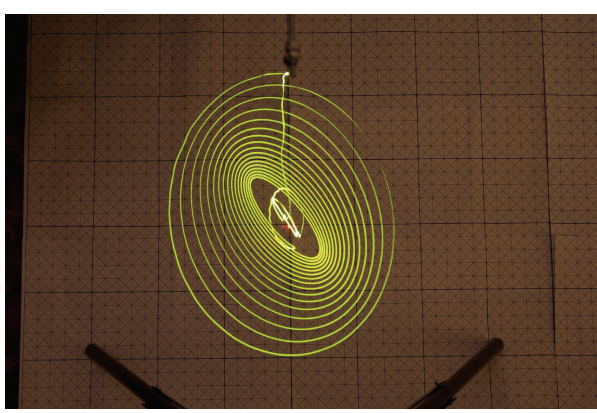

(a)

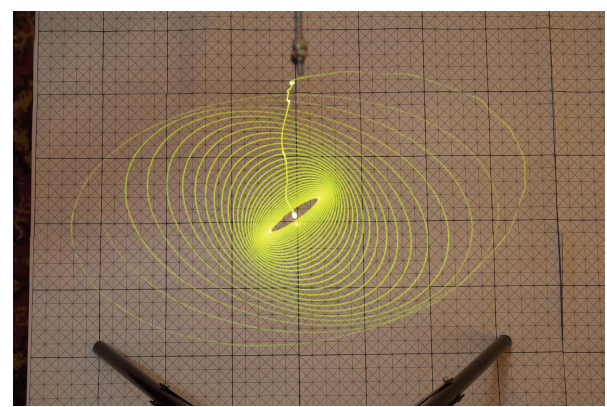

(b)

FIGURE 6: Experimental trajectories of the spherical pendulum with fixed pivot center and fixed cable length $l=0.825 \mathrm{~m}(\mathrm{a} ; \mathrm{b})$.

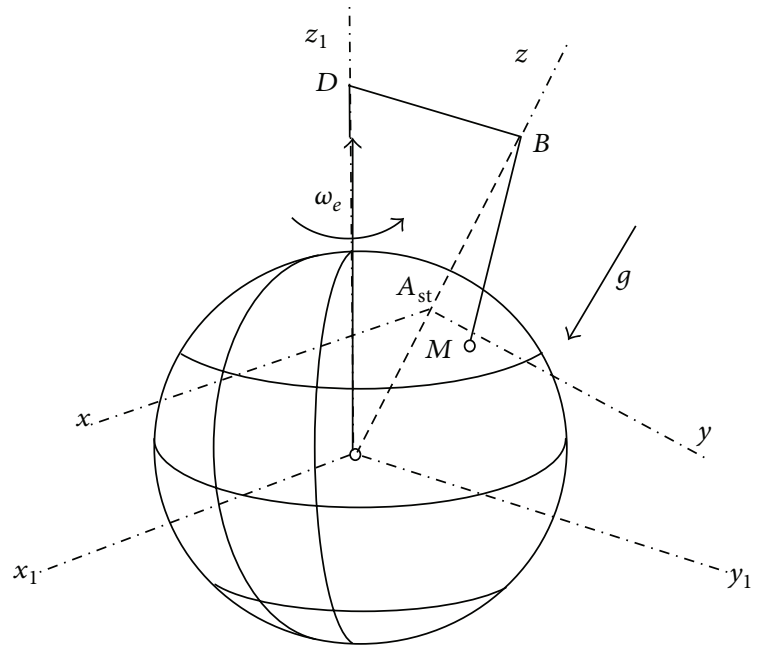

FIGURE 7: Foucault's pendulum schematic view.

It is important to note that the structure of linearized equations (H.4)-(H.5) for the swaying payload during crane boom uniform rotation in the noninertial reference frame $\mathscr{F}$ is analogous to the structure of the governing equations for Foucault's pendulum motion in the known published works by Zhuravlev and Petrov [100] (formula (6), p. 36 of [100]), Pardy [88] (formulae (12)-(14), pp. 850-851 of [88]), and Condurache et al. [77] (formulae (1.1)-(1.3), p. 743-744 of [77]). The above mentioned analogy between linearized equations (H.4)-(H.5) and the governing equations in $[77,88$, $100]$ assumes the geometric analogy of relative swaying trajectories between Foucault's pendulum and the boom-driven pendulum with rotating pivot centers (Figures 1(e), 12, and 16). So there is the geometric analogy between computational relative trajectories of load $M$ swaying during crane boom uniform slewing (Figures 1(e), 12, and 16) and relative trajectories of load $M$ swaying in the plane $\left(A_{\mathrm{st}} x y\right)$ around p. $A_{\mathrm{st}}$ in Figure 7, shown by Zhuravlev and Petrov [100] (Figure 4, p. 39 of [100]), and Condurache and Martinusi [77] (Figure 14 at p. 754 and Figure 16 at p. 755 of [77]). The better understanding of Foucault's pendula dynamics is provided with the study of computational relative trajectories in Figures 1(e), 12, and 16. For Foucault's pendulum (Figure 7) the major semiaxes of relative amplitude extremes have angular velocity values equal to the angular velocity of the Earth diurnal rotation. Moreover, for Foucault's pendulum (Figure 7) the direction of rotation of major semiaxes of relative amplitude extremes is oppositely directed to the direction of the Earth diurnal rotation. All the above mentioned means that the plane $\left(A_{\mathrm{st}} B M\right)$ of Foucault's pendula swaying remains fixed in the heliocentric inertial reference frame $x_{2} y_{2} z_{2}$.

The presented analogy between crane boom-driven payload swaying and Foucault's pendulum motion essentially increases knowledge and awareness of the oscillation processes in such dynamic systems.

\section{Discussion and Mechanical Interpretation of Governing Equations for DAE-Based Nonlinear and Linearized Models}

A governing nonlinear DAE-based system (1) for crane boom uniform slewing has been proposed and numerically solved. A conservative system (1) and (H.4)-(H.5) with components $\Phi_{\text {cor } x}$ and $\Phi_{\text {cor } y}$ has been derived. Both projections $\Phi_{\text {cor } x}$ and $\Phi_{\text {cor } y}$ coincide in the noninertial reference frames $\mathscr{B}$ and $\mathscr{F}$.

The occurrence of the first derivatives $d x / d t ; d y / d t$ of the load $M$ relative coordinates with respect to time in terms $-2 \omega_{e}(d y / d t)$ and $2 \omega_{e}(d x / d t)$ determines that there is no decay of the oscillations in (H.4)-(H.5), and (1) but only redirection of the relative velocity vector $\mathbf{V}_{M / \mathscr{F}}=\mathbf{V}_{\mathbf{r}}$ of the load $M$ in the noninertial reference frames $\mathscr{B}$ and $\mathscr{F}$.

The introduction of a linearized model (H.4)-(H.5) allowed the determination of the natural frequencies $\left(v_{1} \neq\right.$ $\nu_{2}$ ) of free oscillations of payload $M: v_{1}=k+\omega_{e}$ and $v_{2}=k-\omega_{e}$.

It follows from the forms of the relative payload trajectory $y=y(x)$ in the noninertial reference frame $\mathscr{F}$ and the absolute trajectory $y_{2}=y_{2}\left(x_{2}\right)$ in the inertial reference frame $\mathscr{E}$ that the resulting motion of the payload $M$ on the cable $M B$, taking into account the Coriolis inertia force $\Phi_{\text {cor }}$ (D.5), will be the sum of two oscillations with natural frequencies $\nu_{1}$ and $\nu_{2}$, and with periods $T_{1}=2 \pi / \nu_{1}$ and $T_{2}=2 \pi / \nu_{2}$.

It is worth noting that the frequencies $v_{1}$ and $v_{2}$ differ by $2 \omega_{e}$. This means that for the small angle assumption (Appendices H-I) we have $v_{1} \approx v_{2}$ and the trajectory 


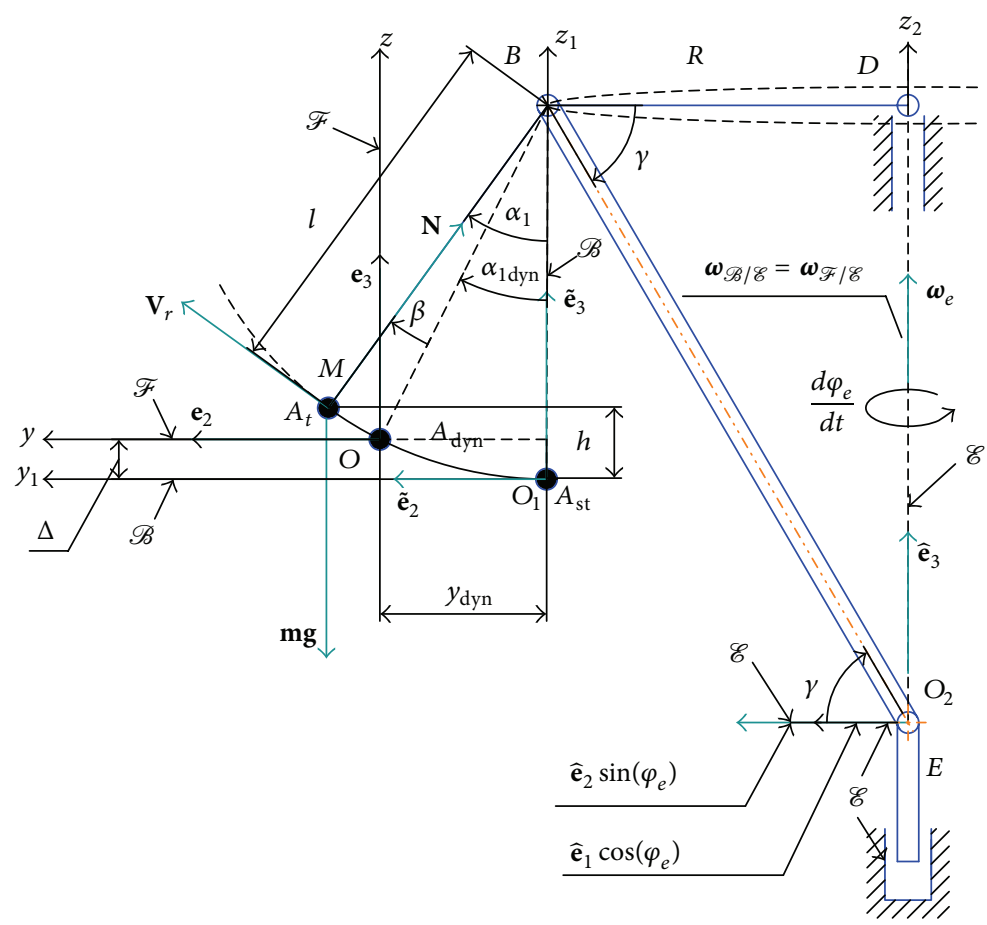

Figure 8: Swaying scheme of load $M$ on the cable $M B$, which is fixedly attached at the point $B$ on the crane boom $B \mathrm{O}_{2}$, in the vertical plane $(y z)$ for the nonlinear model derivation in Cartesian coordinates.

of relative motion $y=y(x)$ in the noninertial reference frame $\mathscr{F}$ on the expiration of the relative oscillations period time $2 \pi / k$ looks approximately like an ellipse (Figures $12(\mathrm{~b})$ and 16(b)) with semiaxes, which are governed by the initial conditions.

The major semiaxis of such an ellipse in Figures 12(b) and 16(b) is defined by the initial velocity of load $M$ in the noninertial reference frame $\mathscr{F}$ and the analytical value of the major semiaxis is equal to $V_{B} / k=\left(\omega_{e} R\right) / k$. The derived value of $V_{B} / k$ is approximately the sum of the absolute values of the amplitudes in the analytical form (I.15) of the linearized Cauchy problem (H.4)-(H.5).

The minor semiaxis of the above mentioned ellipse in Figures $12(\mathrm{~b})$ and $16(\mathrm{~b})$ is defined by the value $y_{\text {dyn }}$, which is approximately the difference of amplitudes in the analytical form (I.15) of the linearized Cauchy problem (H.4)(H.5). In this case the major semiaxis is approximately 520 times larger than the minor semiaxis because the angular velocity $\omega_{e}$ of the transport rotation of crane boom $B \mathrm{O}_{2}$ is much smaller than the natural frequency $k$ of the spherical oscillations of payload $M$ on the cable $M B$ (Figures 12(b) and $16(b))$.

It is also necessary to note that the major semiaxis of the ellipse in the relative motion rotates with an angular velocity $\omega_{e}$ in the opposite direction of the transport rotation of crane boom $\mathrm{BO}_{2}$ in (Figures 12 and 16).

It follows from Figures 1(f), 2, 4, and 5 that the trajectory of absolute motion of load $M$ in the inertial reference frame $\mathscr{E}$ is almost a symmetric curve with $y_{2}$ axial symmetry. The initial and the final motions of load $M$ for half-period essentially differ from its harmonic oscillations neighbor for quarter-period.

Due to the negligible quantity of $\alpha_{\text {dyn }}$ and $y_{\text {dyn }}$ (G.3)(G.5), the average deviation of the load $M$ from the mechanical trajectory of the boom tip $B$ is negligible (Figures 1(f), 2, 4 and 5). The basic dynamic load on the system "crane boom $\mathrm{BO}_{2}$-load $M$ " is created by the high-frequency oscillations of load $M$, which are determined by the action of inertia force $\Phi_{\text {cor }}=-2 m\left(\boldsymbol{\omega}_{\mathscr{B} / \mathscr{E}} \times \mathbf{V}_{M / \mathscr{B}}\right)$, stipulated by the Coriolis acceleration $\mathbf{a}_{\text {cor }}=2 \boldsymbol{\omega}_{\mathscr{B} / \mathscr{C}} \times \mathbf{V}_{M / \mathscr{B}}$ of load $M$ in the noninertial reference frame $\mathscr{F}$. The basic dynamic load in the system defines additional loads and vibrations of crane boom $\mathrm{BO}_{2}$ mechanical elements and support bearings, complicates the automatic and manual control systems of the electromechanical crane boom $\mathrm{BO}_{2}$, and also makes crane operation much more difficult.

It is also important to note that the stop of crane boom $\mathrm{BO}_{2}$ does not lead to instantaneous damping of the load $M$ absolute oscillations in the inertial reference frame $\mathscr{E}$ (Figures 2, 4, and 5). This phenomenon directly follows from the experimental trajectory in Figures 2, 4, and 5. Also, the natural spherical oscillations of load $M$ will occur with the frequency $k$ and the amplitude (the difference between the final position of load $M$ in the relative motion in the noninertial reference frame $\mathscr{F}$ for half-period of vibration and static equilibrium $A_{\text {st }}$ of load $M$ on the cable). The further oscillations for the stop of the crane boom $\mathrm{BO}_{2}$ slewing motion are the deviation of the real trajectory from the intended final position of load $M$. The results of physical simulation in Figures 2, 4, and 5 show the necessity of add-on 


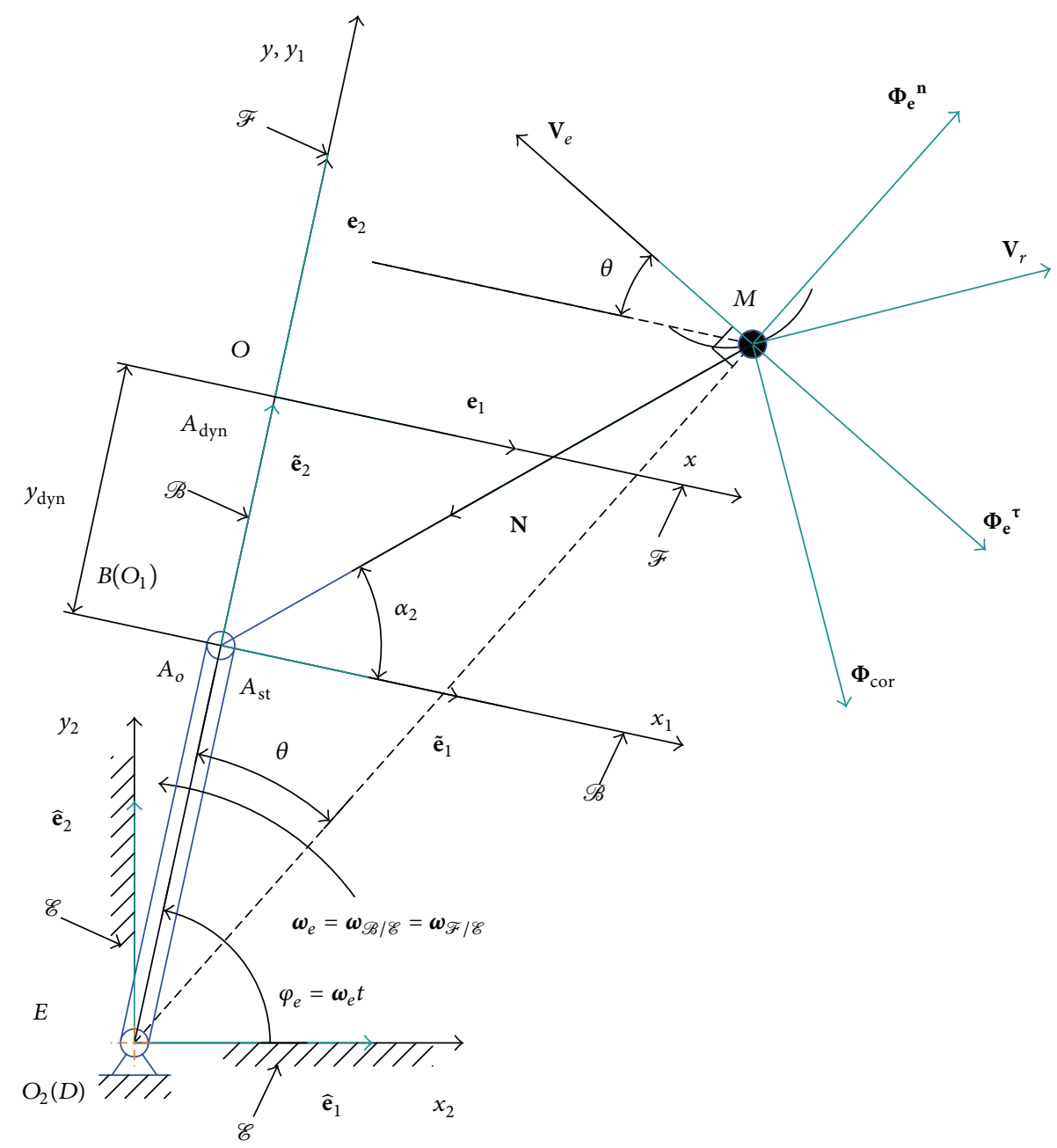

Figure 9: Swaying scheme of load $M$ at the cable $M B$, which is fixedly attached in the point $B$ of the crane boom $B O_{2}$, in the horizontal plane $\left(x_{1} y_{1}\right)$ for the nonlinear model derivation in Cartesian coordinates.

devices development for the efficient suppression of load $M$ final oscillations.

\section{Discussion and Comparison of Derived and Known Published Results}

In Figure 1 and pp. 537-538 of published work by Sakawa et al., 1981 [63], the small angle between payload's cable and vertical line has been introduced, that confirms proposed nonlinear DAE-based system (1) and linearized model (H.4)-(H.5).

In Figure 9 of p. 278 of published work by Maczynski and Wojciech, 2003 [44], the computational finite element method (FEM)-based results were shown for the absolute payload trajectories in the inertial reference frame $\mathscr{E}$. Maczynski's Figure 9 in [44] outlines that the angle between the payload's cable and the vertical line does not exceed 0.1 rad and that agrees with the proposed linearized model (H.4)(H.5) for a small angle assumption. Computational trajectory for Maczynski-derived small load swaying [44] after crane boom stop qualitatively coincides with the experimentally observable load $M$ motion in Figures 2, 4, and 5.
In Figure 6 of published work by Ju et al., 2006 [34], it was shown that the angle between the payload's cable and the vertical line does not exceed $16^{\circ}$. Ju's formula (15a) in p. 382 of [34] assumes that Ju's angle between payload's cable and the vertical line has the harmonic law of variation with an introduction of a small perturbation term. Both Ju's assumptions in [34] confirm the proposed linearized model (H.4)-(H.5).

Comparison of the derived linearized model (H.4)-(H.5) with Figure 5 of published work by Mitrev and Grigorov 2008 in [49] shows that the Mitrev-derived ranges of payload swaying angles within Mitrev's nonlinear model does not exceed $4.8^{\circ}$. Such small values of swaying angles completely confirm the applicability and correctness of the small angle assumption in (H.4)-(H.5).

\section{Final Conclusions}

A governing nonlinear DAE-based system for crane boom uniform slewing has been proposed, numerically solved, and experimentally verified. 
Fully nonlinear differential equations for 3 DOF spherical pendulum oscillations with a uniform slewing crane boom around a fixed vertical axis of rotation have been derived in relative Cartesian and spherical coordinates. The identical linearized differential equations in relative Cartesian coordinates have been derived with an introduction of the Coriolis dynamic theorem and Lagrange equations for a uniform crane boom rotation and small swaying angle $\alpha_{1}$ assumptions.

Linearized and nonlinear theoretical problems for relative swaying of the payload have been formulated in the form of the initial value (Cauchy) problem.

An analytical solution of the linearized system has been derived.

The influences of inertia forces from the centripetal and compound accelerations have been estimated.

A linearized conservative system, which contains first time derivatives of coordinates and no damping of oscillations, has been derived.

The amplitudes of load oscillations, which depend on computed initial conditions, have been estimated within a small angle assumption. The dependence of natural frequencies on the transport inertia forces and gravity forces has been computed for the linearized systems.

The formulae for the association of relative payload motion in the noninertial reference frame $\mathscr{F}$ and absolute payload motion in the inertial reference frame $\mathscr{E}$ have been outlined.

The results of the numerical DAE-based investigation and the performed physical simulation show a satisfactory fit for frequencies and amplitudes of load oscillations.

The dynamic analogy between crane boom-driven payload swaying motion and Foucault's pendulum motion has been grounded and outlined.

The results of the present work are the foundation for further investigations of payload $M$ swaying dynamics during telescopic crane boom nonuniform slewing motion with variable cable length and for the different motions of the pendulum pivot center.

\section{Appendices}

\section{A. Velocity Kinematics Analysis in Cartesian Coordinates}

For the nonlinear problem definition we study the cooperative motion of the mechanical system "crane boom $\mathrm{BO}_{2}$-load $M$ " which is shown in Figures 8-10.

In the nomenclature chapter we denote the fixed inertial frame of reference $\mathscr{E}$ as $x_{2} y_{2} z_{2}$, and the moving noninertial frame of reference $\mathscr{B}$ as $x_{1} y_{1} z_{1}$, which is rigidly bounded with the crane boom $\mathrm{BO}_{2}$. Rotation of the moving noninertial frame of reference $\mathscr{B}\left(x_{1} y_{1} z_{1}\right)$ around the fixed inertial frame of reference $\mathscr{E}\left(x_{2} y_{2} z_{2}\right)$ defines the transportation motion for payload $M$. The motion of load $M$ relative to the moving noninertial frame of reference $\mathscr{B}\left(x_{1} y_{1} z_{1}\right)$ defines the relative motion of payload $M$. The point $A_{\text {st }}$ with the coordinates $x_{1}=0 ; y_{1}=0$; and $z_{1}=0$ is the steady equilibrium position for the load $M$, when the crane boom $B \mathrm{O}_{2}$ is fixed. The point $A_{\text {dyn }}$ with the coordinates $x_{1}=0 ; y_{1}=y_{\mathrm{dyn}}$; and $z_{1}=\Delta$ is the dynamic equilibrium position for the load $M$ for the rotating crane boom $\mathrm{BO}_{2}$. We will assume the point $A_{\text {dyn }}$ as the origin of the noninertial reference frame $\mathscr{F}(x y z)$. The directions of axes $x, y, z$ of the noninertial reference frame $\mathscr{F}$ are parallel to the axes $x_{1}, y_{1}, z_{1}$ of the noninertial reference frame $\mathscr{B}$ in Figures $8-10$. The load $M$ relative motion takes place along the sphere surface with the fixed radius, equal to the cable length $B M=l$.

The computational scheme in Figures 8-10 for the nonlinear model derivation may be described with an introduction of three degrees of freedom. For generalized coordinates we assume the rectangular coordinates $x_{1}, y_{1}$, and $z_{1}$ of the load $M$ in the moving noninertial frame of reference $\mathscr{B}$ and the angle $\varphi_{e}$ of crane boom $B \mathrm{O}_{2}$ slewing in the horizontal plane $\left(x_{1} y_{1}\right)$ with the angular velocity $\omega_{e}$ around the vertical axis $\mathrm{O}_{2} z_{2}$.

The relative velocity vector $\mathbf{V}_{\mathbf{r}}=\mathbf{V}_{M / \mathscr{B}}$ of the load $M$ is defined as $\mathbf{V}_{\mathbf{r}}=\mathbf{V}_{M / \mathscr{B}}=\left(d x_{1} / d t, d y_{1} / d t, d z_{1} / d t\right)$.

In order to derive the absolute velocity $\mathbf{V}_{M / \mathscr{E}}$ of payload $M$ we will apply the vector method for absolute motion assignment. We will define $\mathbf{r}_{\mathrm{O}_{2} / M}$ as the position vector, connecting initial point $\mathrm{O}_{2}$ and terminal point $M$ in noninertial reference frame $\mathscr{B}(m)$; consider

$$
\mathbf{r}_{\mathrm{O}_{2} / M}=\mathbf{r}_{\mathrm{O}_{2} / B}+\mathbf{r}_{B / M}
$$

where vector components in (1) are defined in nomenclature.

In accordance with Figures 8-10 we have the following unit vectors' expansions for position vectors $\mathbf{r}_{\mathrm{O}_{2} / B}$ and $\mathbf{r}_{B / M}$ in (A.1):

$$
\begin{gathered}
\mathbf{r}_{\mathrm{O}_{2} / B}=\left(R \cos \left(\varphi_{e}\right)\right) \widehat{\mathbf{e}}_{1}+\left(R \sin \left(\varphi_{e}\right)\right) \widehat{\mathbf{e}}_{2}+(R \tan (\gamma)) \widehat{\mathbf{e}}_{3} ; \\
\mathbf{r}_{B / M}=x_{1} \widetilde{\mathbf{e}}_{1}+y_{1} \widetilde{\mathbf{e}}_{2}+\left(z_{1}-l\right) \widetilde{\mathbf{e}}_{3},
\end{gathered}
$$

where $R, \varphi_{e}, x_{1}$, and $y_{1}$ notations have been defined in nomenclature and in Figures 8-10.

For further definition of the position vector $\mathbf{r}_{\mathrm{O}_{2} / M}$ in inertial reference frame $\mathscr{E}$, we write unit vectors of noninertial reference frame $\mathscr{B}$ in (A.3) through the unit vectors of inertial reference frame $\mathscr{E}$ :

$$
\begin{aligned}
& \widetilde{\mathbf{e}}_{1}=\left(\sin \left(\varphi_{e}\right)\right) \widehat{\mathbf{e}}_{1}-\left(\cos \left(\varphi_{e}\right)\right) \widehat{\mathbf{e}}_{2} ; \\
& \widetilde{\mathbf{e}}_{2}=\left(\cos \left(\varphi_{e}\right)\right) \widehat{\mathbf{e}}_{1}-\left(\sin \left(\varphi_{e}\right)\right) \widehat{\mathbf{e}}_{2} ; \\
& \widetilde{\mathbf{e}}_{3}=\widehat{\mathbf{e}}_{3} .
\end{aligned}
$$

After substitution of formulae (A.2)-(A.6) in (A.1) and some algebraic manipulations the vector expression (A.1) for the position vector $\mathbf{r}_{\mathrm{O}_{2} / M}$ in the inertial reference frame $\mathscr{E}$ will take the following form:

$$
\begin{aligned}
\mathbf{r}_{\mathrm{O}_{2} / M}= & \left(\left(R+y_{1}\right) \cos \left(\varphi_{e}\right)+x_{1} \sin \left(\varphi_{e}\right)\right) \widehat{\mathbf{e}}_{1} \\
& +\left(\left(R+y_{1}\right) \sin \left(\varphi_{e}\right)-x_{1} \cos \left(\varphi_{e}\right)\right) \widehat{\mathbf{e}}_{2} \\
& +\left(R \tan (\gamma)+z_{1}-l\right) \widehat{\mathbf{e}}_{3} .
\end{aligned}
$$


The absolute velocity of payload $M$ we define by time differentiation of (A.7) assuming constancy of unit vectors $\widehat{\mathbf{e}}_{1}, \widehat{\mathbf{e}}_{2}$, and $\widehat{\mathbf{e}}_{3}$ of the inertial reference frame $\mathscr{E}$ and constancy $R$ and $\gamma$ :

$$
\mathbf{V}_{M / \mathscr{E}}=\mathbf{V}_{\mathrm{abs}}=\frac{d\left(\mathbf{r}_{\mathrm{O}_{2} / M}\right)}{d t}
$$

After differentiation and algebraic transformations in (A.7) and (A.8) we have the following vector expression for absolute payload $M$ velocity in the inertial reference frame $\mathscr{E}:$

$$
\begin{aligned}
\mathbf{V}_{M / \mathscr{E}} & \left(\left(\left(\frac{d y_{1}}{d t}\right)+x_{1}\left(\frac{d \varphi_{e}}{d t}\right)\right) \cos \left(\varphi_{e}\right)\right. \\
+ & \left.\left(\left(\frac{d x_{1}}{d t}\right)-\left(R+y_{1}\right)\left(\frac{d \varphi_{e}}{d t}\right)\right) \sin \left(\varphi_{e}\right)\right) \widehat{\mathbf{e}}_{1} \\
+ & \left(\left(\left(\frac{d y_{1}}{d t}\right)+x_{1}\left(\frac{d \varphi_{e}}{d t}\right)\right) \sin \left(\varphi_{e}\right)\right. \\
& \left.+\left(\left(R+y_{1}\right)\left(\frac{d \varphi_{e}}{d t}\right)-\left(\frac{d x_{1}}{d t}\right)\right) \cos \left(\varphi_{e}\right)\right) \\
\times & \widehat{\mathbf{e}}_{2}+\left(\frac{d z_{1}}{d t}\right) \widehat{\mathbf{e}}_{3} .
\end{aligned}
$$

The square of absolute payload $M$ velocity may be written as

$$
\begin{aligned}
V_{\mathrm{abs}}^{2}= & \left(\frac{d x_{1}}{d t}\right)^{2}+\left(\frac{d y_{1}}{d t}\right)^{2}+\left(\frac{d z_{1}}{d t}\right)^{2}+\left(\frac{d \varphi_{e}}{d t}\right)^{2} \\
& \times\left(x_{1}^{2}+\left(y_{1}+R\right)^{2}\right)+2\left(\frac{d \varphi_{e}}{d t}\right) \\
& \times\left(x_{1}\left(\frac{d y_{1}}{d t}\right)-\left(y_{1}+R\right)\left(\frac{d x_{1}}{d t}\right)\right) .
\end{aligned}
$$

After algebraic transformations we have the square of absolute payload $M$ velocity in the form of

$$
\begin{aligned}
\left(\mathbf{V}_{M / \mathscr{E}}, \mathbf{V}_{M / \mathscr{E}}\right)= & V_{\mathrm{abs}}^{2} \\
= & \left(\left(\frac{d x_{1}}{d t}\right)-\left(\frac{d \varphi_{e}}{d t}\right)\left(R+y_{1}\right)\right)^{2} \\
& +\left(\left(\frac{d y_{1}}{d t}\right)+\left(\frac{d \varphi_{e}}{d t}\right) x_{1}\right)^{2}+\left(\frac{d z_{1}}{d t}\right)^{2} .
\end{aligned}
$$

Algebraic expressions (A.10) and (A.11) could be derived on the basis of a velocity addition theorem for payload $M$ compound motion in the inertial reference frame $\mathscr{E}$ through the unit vectors of the noninertial reference frame $\mathscr{B}$ (see $[59,76,81,82,84,85,87,90-92,94-98])$ :

$$
\mathbf{V}_{M / \mathscr{E}}=\mathbf{V}_{M / \mathscr{B}}+\mathbf{V}_{\mathrm{O}_{2} / \mathscr{E}}+\boldsymbol{\omega}_{\mathscr{B} / \mathscr{E}} \times \mathbf{r}_{\mathrm{O}_{2} / M}
$$

where payload relative velocity in the noninertial reference frame $\mathscr{B}$ may be written as

$$
\mathbf{V}_{M / \mathscr{B}}=\mathbf{V}_{\mathbf{r}}=\left(\frac{d x_{1}}{d t}\right) \widetilde{\mathbf{e}}_{1}+\left(\frac{d y_{1}}{d t}\right) \widetilde{\mathbf{e}}_{2}+\left(\frac{d z_{1}}{d t}\right) \widetilde{\mathbf{e}}_{3}
$$

The velocity $\mathbf{V}_{\mathrm{O}_{2} / \mathscr{E}}$ of the point $\mathrm{O}_{2}=$ point $E$ in the inertial reference frame $\mathscr{E}$ has zero value:

$$
\mathbf{V}_{\mathrm{O}_{2} / \mathscr{E}}=0
$$

The last term in (A.12) is the vector product $\boldsymbol{\omega}_{\mathscr{B} / \mathscr{E}} \times$ $\mathbf{r}_{\mathrm{O}_{2} / M}$, where crane boom angular velocity vector is $\boldsymbol{\omega}_{\mathscr{B} / \mathscr{E}}=$ $\left(d \varphi_{e} / d t\right) \widetilde{\mathbf{e}}_{3}$ (A.6) and the position vector $\mathbf{r}_{\mathrm{O}_{2} / M}$ in the noninertial reference frame $\mathscr{B}$ is as follows:

$$
\mathbf{r}_{\mathrm{O}_{2} / M}=x_{1} \widetilde{\mathbf{e}}_{1}+\left(y_{1}+R\right) \widetilde{\mathbf{e}}_{2}+z_{1} \widetilde{\mathbf{e}}_{3}
$$

Taking into account (A.13)-(A.15) the vector equation (A.12) in the inertial reference frame $\mathscr{E}$ written through the unit vectors $\widetilde{\mathbf{e}}_{1}, \widetilde{\mathbf{e}}_{2}, \widetilde{\mathbf{e}}_{3}$ of the noninertial reference frame $\mathscr{B}$ will have the following form:

$$
\begin{aligned}
\mathbf{V}_{M / \mathscr{E}}= & \left(\left(\frac{d x_{1}}{d t}\right)-\left(\frac{d \varphi_{e}}{d t}\right)\left(R+y_{1}\right)\right) \widetilde{\mathbf{e}}_{1} \\
& +\left(\left(\frac{d y_{1}}{d t}\right)+\left(\frac{d \varphi_{e}}{d t}\right) x_{1}\right) \widetilde{\mathbf{e}}_{2}+\left(\frac{d z_{1}}{d t}\right) \widetilde{\mathbf{e}}_{3} .
\end{aligned}
$$

So, on the basis of (A.16), we have the following square of absolute payload $M$ velocity as

$$
\begin{aligned}
\left(\mathbf{V}_{M / \mathscr{C}}, \mathbf{V}_{M / \mathscr{C}}\right)= & V_{\mathrm{abs}}^{2} \\
= & \left(\left(\frac{d x_{1}}{d t}\right)-\left(\frac{d \varphi_{e}}{d t}\right)\left(R+y_{1}\right)\right)^{2} \\
& +\left(\left(\frac{d y_{1}}{d t}\right)+\left(\frac{d \varphi_{e}}{d t}\right) x_{1}\right)^{2}+\left(\frac{d z_{1}}{d t}\right)^{2} .
\end{aligned}
$$

Independently derived expressions for the scalar product $\left(\mathbf{V}_{M / \mathscr{E}}, \mathbf{V}_{M / \mathscr{C}}\right)$ in (A.11) and (A.17) completely coincide, which confirms the correctness and accuracy of the pendulum absolute velocity $\mathbf{V}_{M / \mathscr{E}}$ derivation and shows that the scalar product $\left(\mathbf{V}_{M / \mathscr{C}}, \mathbf{V}_{M / \mathscr{E}}\right)$ is the invariant expression, independent of choice of reference frame.

The second and third terms of (A.12) in the noninertial reference frame $\mathscr{B}$ determine the vector of the load $M$ transportation velocity as

$$
\mathbf{V}_{\mathbf{e}}=\mathbf{V}_{\mathrm{O}_{2} / \mathscr{E}}+\boldsymbol{\omega}_{\mathscr{B} / \mathscr{E}} \times \mathbf{r}_{\mathrm{O}_{2} / M}
$$

Taking into account (A.14) the scalar of the load $M$ transportation velocity is defined as

$$
\left\|\mathbf{V}_{\mathbf{e}}\right\|=\omega_{\mathscr{B} / \mathscr{E}}\left\|\mathbf{r}_{\mathrm{O}_{2} / M}\right\|
$$




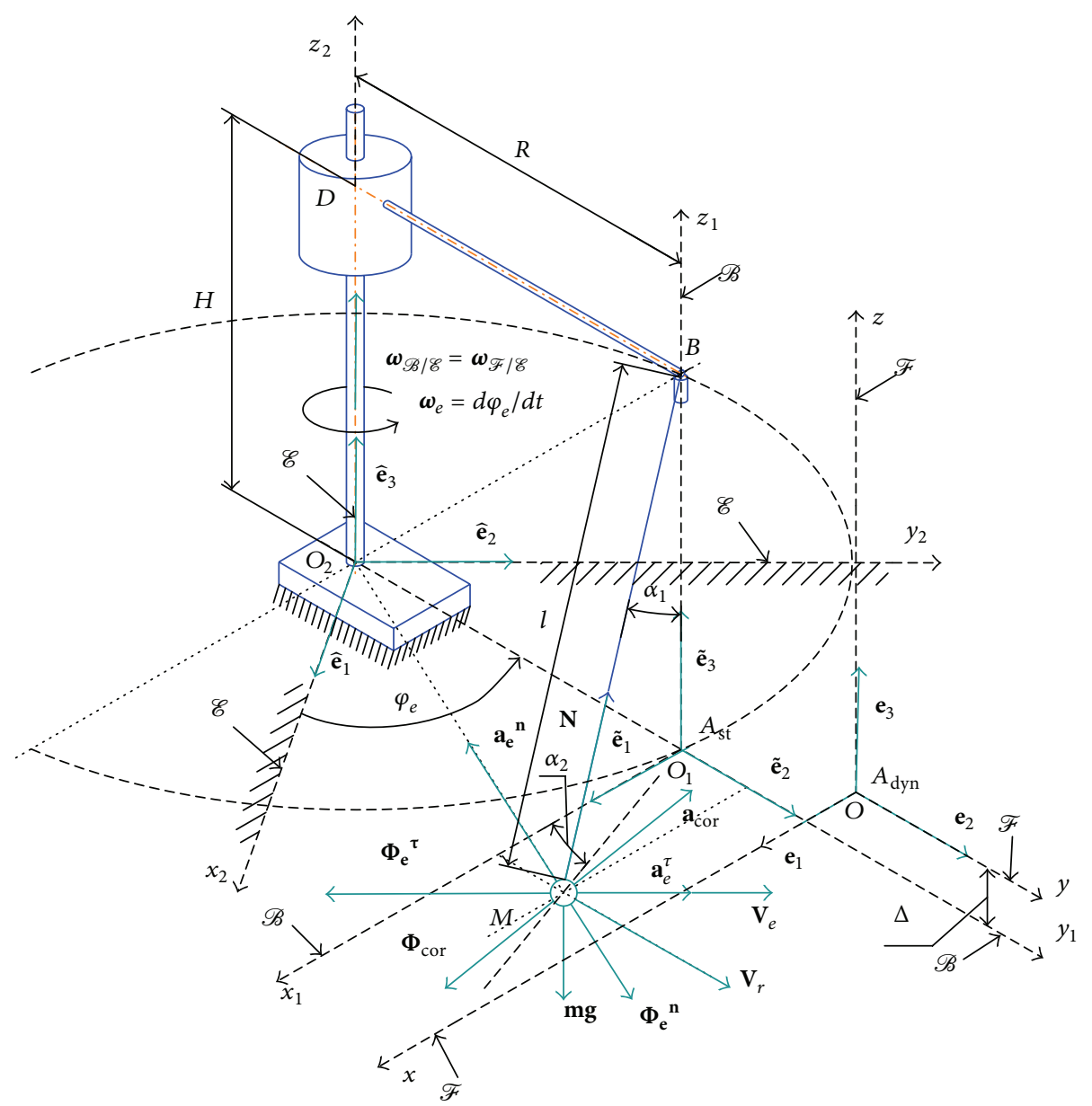

Figure 10: Computational spatial scheme of spherical pendulum $M$, swaying on the cable $M B$ during crane boom $\mathrm{BO}_{2}$ slewing motion for the nonlinear model derivation in Cartesian coordinates.

The transportation velocity vector $\mathbf{V}_{\mathbf{e}}$ is perpendicular to $\mathbf{r}_{\mathrm{O}_{2} / M}$ and $\mathbf{r}_{D / M}$ (Figures 8-10); that is,

$$
\begin{aligned}
& \left(\mathbf{V}_{\mathrm{e}}, \mathbf{r}_{\mathrm{O}_{2} / M}\right)=\left(\mathbf{v}_{\mathrm{O}_{2} / \mathscr{E}}+\boldsymbol{\omega}_{\mathscr{R} / \mathscr{E}} \times \mathbf{r}_{\mathrm{O}_{2} / M}, \mathbf{r}_{\mathrm{O}_{2} / M}\right)=0 ; \\
& \left(\mathbf{V}_{\mathbf{e}}, \mathbf{r}_{D / M}\right)=\left(\mathbf{v}_{\mathrm{O}_{2} / \mathscr{E}}+\boldsymbol{\omega}_{\mathscr{B} / \mathscr{C}} \times \mathbf{r}_{\mathrm{O}_{2} / M}, \mathbf{r}_{D / M}\right)=0 .
\end{aligned}
$$

In nomenclature and in Figure 9 we denote the current angle $\theta=\angle\left(\widetilde{\mathbf{e}}_{2},\left(\mathbf{r}_{\mathrm{O}_{2} / M}\right)_{x_{1} y_{1}}\right)$, where

$$
\sin (\theta)=\frac{x_{1}}{\left\|\mathbf{r}_{\mathrm{O}_{2} / M}\right\|} ; \quad \cos (\theta)=\frac{\left(R+y_{1}\right)}{\left\|\mathbf{r}_{\mathrm{O}_{2} / M}\right\|} .
$$

In the noninertial reference frame $\mathscr{B}$ the vector of the load $M$ transportation velocity $\mathbf{V}_{\mathbf{e}}$ is defined as

$$
\mathbf{V}_{\mathbf{e}}=\left(-\left\|\mathbf{V}_{\mathbf{e}}\right\| \cos (\theta)\right) \widetilde{\mathbf{e}}_{1}+\left(\left\|\mathbf{V}_{\mathbf{e}}\right\| \sin (\theta)\right) \widetilde{\mathbf{e}}_{2} \text {. }
$$
form:

Taking into account (A.19), (A.22) takes the following

$$
\begin{aligned}
\mathbf{V}_{\mathbf{e}}= & \left(-\omega_{\mathscr{B} / \mathscr{C}}\left\|\mathbf{r}_{\mathrm{O}_{2} / M}\right\| \cos (\theta)\right) \widetilde{\mathbf{e}}_{1} \\
& +\left(\omega_{\mathscr{B} / \mathscr{E}}\left\|\mathbf{r}_{\mathrm{O}_{2} / M}\right\| \sin (\theta)\right) \widetilde{\mathbf{e}}_{2} .
\end{aligned}
$$

Assuming (A.21) equation (A.23) will take the following form in the noninertial reference frame $\mathscr{B}$ :

$$
\mathbf{V}_{\mathbf{e}}=\left(-\left(\frac{d \varphi_{e}}{d t}\right)\left(R+y_{1}\right)\right) \widetilde{\mathbf{e}}_{1}+\left(\left(\frac{d \varphi_{e}}{d t}\right) x_{1}\right) \widetilde{\mathbf{e}}_{2} .
$$

Taking into account (A.13) and (A.24), the formula (A.12) yields again (A.16) and (A.17).

So the square of absolute payload $M$ velocity (A.10), (A.11), and (A.17) has been derived with three independent methods, which confirms the accuracy and correctness of expressions (A.10), (A.11), and (A.17).

\section{B. Acceleration Kinematics Analysis}

Further dynamic analysis, with the introduction of Newton's second law, requires us to study the accelerations of payload $M$, shown in Figure 10. 
The standard vector equation for the acceleration addition for payload $M$ in the inertial reference frame $\mathscr{E}$ has the form (see [59, 76, 81, 82, 84, 85, 87, 90-92, 94-98]):

$$
\begin{aligned}
\mathbf{a}_{M / \mathscr{E}}= & \mathbf{a}_{M / \mathscr{B}}+\mathbf{a}_{E / \mathscr{E}}+\boldsymbol{\alpha}_{\mathscr{B} / \mathscr{E}} \times \mathbf{r}_{E / M} \\
& +\boldsymbol{\omega}_{\mathscr{B} / \mathscr{C}} \times\left(\boldsymbol{\omega}_{\mathscr{B} / \mathscr{E}} \times \mathbf{r}_{E / M}\right) \\
& +2 \boldsymbol{\omega}_{\mathscr{B} / \mathscr{C}} \times \mathbf{V}_{M / \mathscr{B}} .
\end{aligned}
$$

In our case we assume point $E$ as the pole for payload transportation motion, located at the vertical axis $\mathrm{O}_{2} z_{2}$. So the second term in (B.1) for the inertial reference frame $\mathscr{E}$ and in the noninertial reference frame $\mathscr{B}$ takes the form

$$
\mathbf{a}_{E / \mathscr{E}}=\mathbf{a}_{E / \mathscr{B}}=0 .
$$

So taking into account the nomenclature and (B.2), (B.1) in the inertial reference frame $\mathscr{E}$ takes the following form:

$$
a_{a b s}=a_{r}+a_{e}^{\tau}+a_{e}{ }^{n}+a_{\text {cor }} .
$$

Equations (B.1) and (B.2) contain the following accelerations.

The vector of payload $M$ relative acceleration is defined in the noninertial reference frame $\mathscr{B}$ as

$$
\begin{aligned}
\mathbf{a}_{\mathbf{r}} & =\mathbf{a}_{M / \mathscr{B}} \\
& =\left(\frac{d^{2} x_{1}}{d t^{2}}\right) \widetilde{\mathbf{e}}_{1}+\left(\frac{d^{2} y_{1}}{d t^{2}}\right) \widetilde{\mathbf{e}}_{2}+\left(\frac{d^{2} z_{1}}{d t^{2}}\right) \widetilde{\mathbf{e}}_{3} .
\end{aligned}
$$

The vector of tangential acceleration $\mathbf{a}_{\mathbf{e}}{ }^{\boldsymbol{\tau}}$ for transportation of payload $M$ has the same direction as the vector of the load $M$ transportation velocity $\mathbf{V}_{\mathbf{e}}$, that is, $\mathbf{a}_{\mathbf{e}}{ }^{\boldsymbol{\tau}} \uparrow \uparrow \mathbf{V}_{\mathbf{e}}$ and is defined in the noninertial reference frame $\mathscr{B}$ as

$$
\begin{aligned}
\mathbf{a}_{\mathbf{e}}{ }^{\boldsymbol{\tau}} & =\boldsymbol{\alpha}_{\mathscr{B} / \mathscr{C}} \times \mathbf{r}_{E / M} \\
& =\left(-\left(\frac{d^{2} \varphi_{e}}{d t^{2}}\right)\left(R+y_{1}\right)\right) \widetilde{\mathbf{e}}_{1}+\left(\left(\frac{d^{2} \varphi_{e}}{d t^{2}}\right) x_{1}\right) \widetilde{\mathbf{e}}_{2} .
\end{aligned}
$$

The vector of the normal or centripetal acceleration $\mathbf{a}_{\mathbf{e}}{ }^{\mathbf{n}}$ of transportation for payload $M$ is directed towards the axis $\mathrm{O}_{2} z_{2}$ and at the same time $\mathbf{a}_{\mathbf{e}}{ }^{\boldsymbol{\tau}}$ and $\mathbf{a}_{\mathbf{e}}{ }^{\mathbf{n}}$ are the coplanar vectors, located in the horizontal plane $\left(x_{2} y_{2}\right)$, where

$$
\begin{aligned}
\mathbf{a}_{\mathbf{e}}{ }^{\mathbf{n}} & =\boldsymbol{\omega}_{\mathscr{B} / \mathscr{E}} \times\left(\boldsymbol{\omega}_{\mathscr{B} / \mathscr{E}} \times \mathbf{r}_{E / M}\right) \\
& =\left(-\left(\frac{d \varphi_{e}}{d t}\right)^{2} x_{1}\right) \widetilde{\mathbf{e}}_{1}-\left(\left(\frac{d \varphi_{e}}{d t}\right)^{2}\left(R+y_{1}\right)\right) \widetilde{\mathbf{e}}_{2}
\end{aligned}
$$

The vector of the Coriolis (compound) acceleration of payload $M$ is directed in accordance with the vector product law

$$
\begin{aligned}
\mathbf{a}_{\text {cor }} & =2 \boldsymbol{\omega}_{\mathscr{B} / \mathscr{E}} \times \mathbf{V}_{M / \mathscr{B}} \\
& =\left(-2\left(\frac{d \varphi_{e}}{d t}\right)\left(\frac{d y_{1}}{d t}\right)\right) \widetilde{\mathbf{e}}_{1}+\left(2\left(\frac{d \varphi_{e}}{d t}\right)\left(\frac{d x_{1}}{d t}\right)\right) \widetilde{\mathbf{e}}_{2} .
\end{aligned}
$$

\section{The Geometric Constraints Imposed on the Payload}

The geometric constraint, imposed on the payload $M$ is shown in Figures 8-10 in the form of the cable BM. The length $l=l_{B M}=\left\|\mathbf{r}_{B / M}\right\|$ of the cable $B M$ determines the geometrical constraint in this problem:

$$
l^{2}=x_{1}^{2}+y_{1}^{2}+\left(z_{1}-l\right)^{2} .
$$

On the basis of Figure 10 we may derive that

$$
\begin{aligned}
l^{2}= & \left(l \sin \left(\alpha_{1}\right) \cos \left(\alpha_{2}\right)\right)^{2} \\
& +\left(l \sin \left(\alpha_{1}\right) \sin \left(\alpha_{2}\right)\right)^{2}+\left(l \cos \left(\alpha_{1}\right)\right)^{2},
\end{aligned}
$$

where angles $\alpha_{1}$ and $\alpha_{2}$ in Figures $8-10$ are the spherical coordinates of spherical pendulum $M$.

The comparison of formulae (C.1) and (C.2) allows us to determine the Cartesian coordinates $x_{1} ; y_{1}$; and $z_{1}$ in the noninertial reference frame $\mathscr{B}$ as

$$
\begin{aligned}
& x_{1}=l \sin \left(\alpha_{1}\right) \cos \left(\alpha_{2}\right) ; \\
& y_{1}=l \sin \left(\alpha_{1}\right) \sin \left(\alpha_{2}\right) ; \\
& z_{1}=l-l \cos \left(\alpha_{1}\right) .
\end{aligned}
$$

The geometric constraint of (C.1)-(C.3) can be derived on the basis of (C.3) as

$$
\begin{gathered}
l^{2} \sin ^{2}\left(\alpha_{1}\right)=x_{1}^{2}+y_{1}^{2} \\
z_{1}=l-l \cos \left(\alpha_{1}\right) .
\end{gathered}
$$

Equations (C.4) yield the following partial derivatives of $\alpha_{1}$ with respect to Cartesian coordinates $x_{1} ; y_{1}$; and $z_{1}$ in the noninertial reference frame $\mathscr{B}$ as

$$
\begin{aligned}
& \frac{\partial \alpha_{1}}{\partial x_{1}}=\frac{x_{1}}{l^{2} \sin \left(\alpha_{1}\right) \cos \left(\alpha_{1}\right)} \\
& \frac{\partial \alpha_{1}}{\partial y_{1}}=\frac{y_{1}}{l^{2} \sin \left(\alpha_{1}\right) \cos \left(\alpha_{1}\right)} \\
& \frac{\partial \alpha_{1}}{\partial z_{1}}=\frac{1}{l \sin \left(\alpha_{1}\right)}
\end{aligned}
$$

Absolute coordinates $x_{2}, y_{2}$, and $z_{2}$ in inertial reference frame $\mathscr{E}$ which depend upon the relative coordinates $x_{1}, y_{1}$, and $z_{1}$ in noninertial reference frame $\mathscr{B}$ during the swaying of load $M$ are defined according to the following equations (Figures 8-10):

$$
\begin{aligned}
& x_{2}=\left(R+y_{1}\right) \cos \left(\varphi_{e}\right)+x_{1} \sin \left(\varphi_{e}\right) ; \\
& y_{2}=\left(R+y_{1}\right) \sin \left(\varphi_{e}\right)-x_{1} \cos \left(\varphi_{e}\right) ; \\
& z_{2}=z_{1}=l-l \cos \left(\alpha_{1}\right) .
\end{aligned}
$$




\section{Forces Imposed on the Payload}

Among the forces imposed on the payload $M$ (Figure 3(a)) we have an active force of gravity $\mathbf{m g}$, the cable reaction force $\mathbf{N}$, the tangential inertial force $\Phi_{\mathrm{e}}{ }^{\tau}$, the normal or centrifugal inertial force $\boldsymbol{\Phi}_{\mathrm{e}}{ }^{\mathbf{n}}$, and the Coriolis inertial force $\boldsymbol{\Phi}_{\text {cor }}$. Taking into account formulae (B.1)-(B.7) we will express below all imposed forces in the noninertial reference frame $\mathscr{B}$

$$
\begin{aligned}
& \mathbf{m g}=(-m g) \widetilde{\mathbf{e}}_{3} ; \\
& \mathbf{N}=-N\left(\frac{x_{1}}{l}\right) \widetilde{\mathbf{e}}_{1}-N\left(\frac{y_{1}}{l}\right) \widetilde{\mathbf{e}}_{2}-N\left(\frac{\left(z_{1}-l\right)}{l}\right) \widetilde{\mathbf{e}}_{3} ; \\
& \boldsymbol{\Phi}_{\mathbf{e}} \mathbf{n}^{\mathbf{n}}=(-m) \mathbf{a}_{\mathbf{e}}{ }^{\mathbf{n}}=\left(m\left(\frac{d \varphi_{e}}{d t}\right)^{2} x_{1}\right) \widetilde{\mathbf{e}}_{1} \\
& +\left(m\left(\frac{d \varphi_{e}}{d t}\right)^{2}\left(R+y_{1}\right)\right) \widetilde{\mathbf{e}}_{2} ; \\
& \boldsymbol{\Phi}_{\mathbf{e}}{ }^{\tau}=(-m) \mathbf{a}_{\mathbf{e}}{ }^{\boldsymbol{\tau}}=\left(m\left(\frac{d^{2} \varphi_{e}}{d t^{2}}\right)\left(R+y_{1}\right)\right) \widetilde{\mathbf{e}}_{1} \\
& -\left(m\left(\frac{d^{2} \varphi_{e}}{d t^{2}}\right) x_{1}\right) \widetilde{\mathbf{e}}_{2} ; \\
& \boldsymbol{\Phi}_{\text {cor }}=(-m) \mathbf{a}_{\text {cor }}=\left(2 m\left(\frac{d \varphi_{e}}{d t}\right)\left(\frac{d y_{1}}{d t}\right)\right) \widetilde{\mathbf{e}}_{1} \\
& -\left(2 m\left(\frac{d \varphi_{e}}{d t}\right)\left(\frac{d x_{1}}{d t}\right)\right) \widetilde{\mathbf{e}}_{2},
\end{aligned}
$$

where $-\left(x_{1} / l\right) ;-\left(y_{1} / l\right)$ and $-\left(\left(z_{1}-l\right) / l\right)$ are the direction cosines of the cable reaction force $\mathbf{N}$ in the noninertial reference frame $\mathscr{B}$. The force $\mathbf{N}$ is directed from point $M$ to point $\mathscr{B}$; that is, the force $\mathbf{N} \uparrow \downarrow \mathbf{r}_{B / M}$ is oppositely directed to the $\mathbf{r}_{B / M}$ (A.3).

\section{E. Forces Imposed on the Crane Boom-Payload System}

The slewing motion of the mechanical system "crane boom $\mathrm{BO}_{2}$-load $\mathrm{M}$ " in Figures 8-10 is governed by the vector equation for the rate of change of moment of momentum $\mathbf{H}_{3}^{\mathrm{O}_{2}}$ for the system "crane boom $\mathrm{BO}_{2}$-load $M$ " with respect to point $\mathrm{O}_{2}$ in the inertial reference frame $\mathscr{E}$ :

$$
\frac{{ }^{\mathscr{E}} d \mathbf{H}_{3}^{\mathrm{O}_{2}}}{d t}=\sum \mathbf{M}^{\mathrm{O}_{2}} .
$$
nents:

The vector equation (E.1) contains the following compo-

$$
\begin{gathered}
\mathbf{H}_{3}^{\mathrm{O}_{2}}=I_{33}^{\mathrm{O}_{2}} \boldsymbol{\omega}_{\mathscr{B} / \mathscr{E}} ; \\
I_{33}^{\mathrm{O}_{2}}=\left(I_{33}^{\mathrm{O}_{2}}\right)_{B \mathrm{O}_{2}}+m\left(x_{1}^{2}+\left(R+y_{1}\right)^{2}\right) ; \\
\sum \mathbf{M}^{\mathrm{O}_{2}}=\mathbf{M}_{D T}^{\mathrm{O}_{2}}-\mathbf{M}_{F T}^{\mathrm{O}_{2}}=\left(M_{D T}^{\mathrm{O}_{2}}-M_{F T}^{\mathrm{O}_{2}}\right) \widehat{\mathbf{e}}_{3},
\end{gathered}
$$

where $\left(I_{33}^{\mathrm{O}_{2}}\right)_{\mathrm{BO}_{2}}$ is the element of mass moment of inertia for the crane boom $\mathrm{BO}_{2}$ in inertial fixed on earth reference frame $\mathscr{E}$ with respect to axis $\widehat{\mathbf{e}}_{3} ; m\left(x_{1}^{2}+\left(R+y_{1}\right)^{2}\right)$ is the element of mass moment of inertia for the payload $M$ in inertial fixed on earth reference frame $\mathscr{E}$ with respect to axis $\widehat{\mathbf{e}}_{3}$.

The external moment of gravitational force $\mathbf{M}^{\mathrm{O}_{2}}(\mathbf{m g})=0$ in (E.4) because $\mathbf{m g} \uparrow \downarrow \widehat{\mathbf{e}}_{3}$.

For the system "crane boom-payload" the cable reaction force $\mathbf{N}$ is the internal force. So in (E.1) and (E.4) we have $\mathbf{M}^{\mathrm{O}_{2}}(\mathbf{N})=0$.

Substitution of (E.2), (E.3), and (E.4) into (E.1) yields the following scalar equation for the rate of change of moment of momentum $\mathrm{H}_{3}^{\mathrm{O}_{2}}$ for the system "crane boom $\mathrm{BO}_{2}$-load $\mathrm{M}$ " with respect to point $\mathrm{O}_{2}$ in the inertial reference frame $\mathscr{E}$ :

$$
\begin{aligned}
& \frac{d}{d t}\left(\left(\left(I_{33}^{\mathrm{O}_{2}}\right)_{\mathrm{BO}_{2}}+m\left(x_{1}^{2}+\left(R+y_{1}\right)^{2}\right)\right)\left(\frac{d \varphi_{e}}{d t}\right)\right) \\
& =M_{D T}^{\mathrm{O}_{2}}-M_{F T}^{\mathrm{O}_{2}},
\end{aligned}
$$

where driving $M_{D T}^{\mathrm{O}_{2}}$ and frictional $M_{F T}^{\mathrm{O}_{2}}$ torques (see nomenclature section) are the technically defined functions for specific electric drive systems (see [1-3, 42-44, 59, 63, 67, 76, $81,82,84,85,87,90-92,94-98]$ ).

\section{F. Derivation of the Fully Nonlinear Equations in Relative Cartesian Coordinates of the Noninertial Reference frame $\mathscr{B}$}

The vector differential equation for relative motion of payload $M$ in the noninertial reference frame $\mathscr{B}$ is as follows:

$$
\begin{aligned}
& m \mathbf{a}_{M / \mathscr{B}}=\mathbf{m g}+\mathbf{N}+\boldsymbol{\Phi}_{\mathbf{e}}{ }^{\mathbf{n}}+\boldsymbol{\Phi}_{\mathbf{e}}{ }^{\boldsymbol{\tau}}+\boldsymbol{\Phi}_{\text {cor }} ; \\
m \mathbf{a}_{M / \mathscr{B}}= & \mathbf{m g}+\mathbf{N}+(-m)\left(\boldsymbol{\omega}_{\mathscr{B} / \mathscr{E}} \times\left(\boldsymbol{\omega}_{\mathscr{B} / \mathscr{E}} \times \mathbf{r}_{E / M}\right)\right) \\
& +(-m)\left(\boldsymbol{\alpha}_{\mathscr{B} / \mathscr{C}} \times \mathbf{r}_{E / M}\right)+(-m)\left(2 \boldsymbol{\omega}_{\mathscr{B} / \mathscr{C}} \times \mathbf{V}_{M / \mathscr{B}}\right) .
\end{aligned}
$$

The vector differential equation (F.1)-(F.2) yields three scalar ordinary differential equations (ODEs) for payload $M$ swaying motion.

We will project (F.1) and (F.2) to the axes $x_{1}, y_{1}$, and $z_{1}$ in the noninertial reference frame $\mathscr{B}$

$$
\begin{aligned}
& m\left(\frac{d^{2} x_{1}}{d t^{2}}\right)=-N\left(\frac{x_{1}}{l}\right)+m\left(\frac{d \varphi_{e}}{d t}\right)^{2} x_{1} \\
&+m\left(\frac{d^{2} \varphi_{e}}{d t^{2}}\right)\left(R+y_{1}\right)+2 m\left(\frac{d \varphi_{e}}{d t}\right)\left(\frac{d y_{1}}{d t}\right) \\
& m\left(\frac{d^{2} y_{1}}{d t^{2}}\right)=-N\left(\frac{y_{1}}{l}\right)+m\left(\frac{d \varphi_{e}}{d t}\right)^{2}\left(R+y_{1}\right) \\
&-m\left(\frac{d^{2} \varphi_{e}}{d t^{2}}\right) x_{1}-2 m\left(\frac{d \varphi_{e}}{d t}\right)\left(\frac{d x_{1}}{d t}\right) \\
& m\left(\frac{d^{2} z_{1}}{d t^{2}}\right)=-m g+N\left(\frac{l-z_{1}}{l}\right) .
\end{aligned}
$$




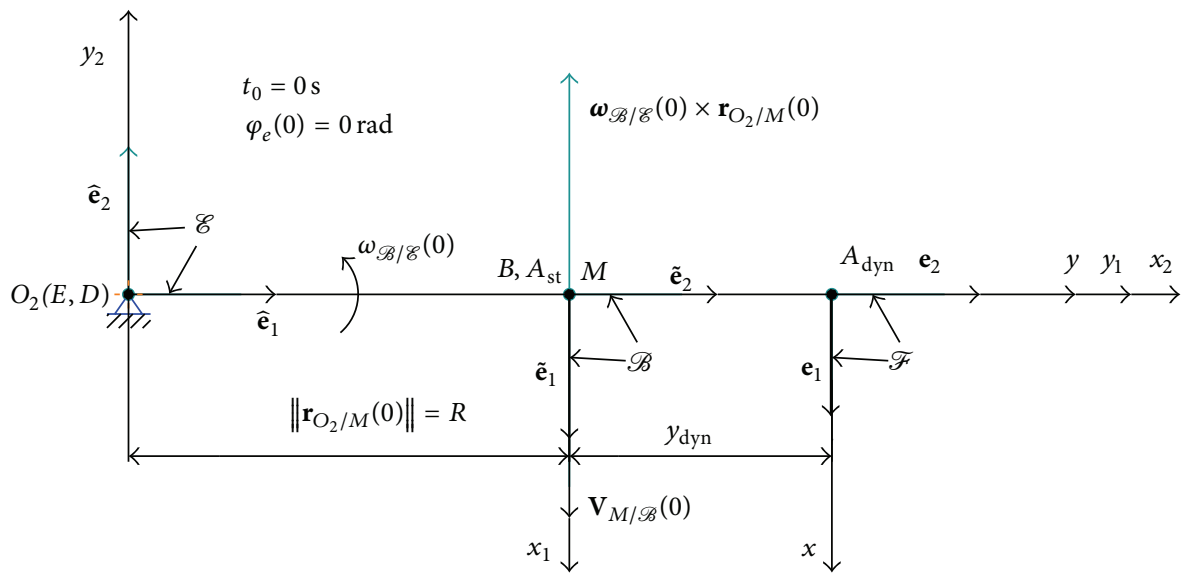

FIGURE 11: The computational scheme for initial conditions assignment.

The derived system (F.3) and (E.5) is the nonlinear ODE system. The nonlinearity of (F.3) is determined by the presence of the unknown function $N=N(t)$, variable boom slewing angle $\varphi_{e}$, variable boom slewing angular velocity $d \varphi_{e} / d t$, and variable angular acceleration $d^{2} \varphi_{e} / d t^{2}$.

In order to verify the correctness of the derived system (F.3) we will utilize second-kind Lagrange equations. Taking into account equations (A.10), (A.11), and (A.17) for the square of absolute payload $M$ velocity $\left(\mathbf{V}_{M / \mathscr{E}}, \mathbf{V}_{M / \mathscr{C}}\right)$, and by adding the kinetic energy (see $[59,76,81,82,84,85,87,90-$ 92, 94-98]) for a slewing crane boom according to (E.2)(E.3), we will have the following expression for "crane boompayload" kinetic energy:

$$
\begin{aligned}
T=\frac{m}{2} & \left(\left(\left(\frac{d x_{1}}{d t}\right)-\left(\frac{d \varphi_{e}}{d t}\right)\left(R+y_{1}\right)\right)^{2}\right. \\
& \left.+\left(\left(\frac{d y_{1}}{d t}\right)+\left(\frac{d \varphi_{e}}{d t}\right) x_{1}\right)^{2}+\left(\frac{d z_{1}}{d t}\right)^{2}\right) \\
+ & \frac{I_{33}^{\mathrm{O}_{2}}}{2}\left(\frac{d \varphi_{e}}{d t}\right)^{2} .
\end{aligned}
$$

Taking into account the nonlinearity and nonconservatism of the cable reaction force $\mathbf{N}$, and the equations for geometric constraints (C.1)-(C.5), we have the following formulae for the generalized forces in the noninertial reference frame $\mathscr{B}$ :

$$
\begin{aligned}
& Q_{x_{1}}=-N\left(\frac{x_{1}}{l}\right) ; \\
& Q_{y_{1}}=-N\left(\frac{y_{1}}{l}\right) ; \\
& Q_{z_{1}}=+N\left(\frac{l-z_{1}}{l}\right)-m g .
\end{aligned}
$$

We will derive the same nonlinear differential equations (F.3) for relative system motion with an introduction of the following Lagrange equations (see $[59,76,81,82,84,85$, $87,90-92,94-98]$ ) in the noninertial reference frame $\mathscr{B}$ :

$$
\begin{aligned}
& \frac{d}{d t}\left(\frac{\partial T}{\partial \dot{x}_{1}}\right)-\frac{\partial T}{\partial x_{1}}=Q_{x_{1}} ; \\
& \frac{d}{d t}\left(\frac{\partial T}{\partial \dot{y}_{1}}\right)-\frac{\partial T}{\partial y_{1}}=Q_{y_{1}} ; \\
& \frac{d}{d t}\left(\frac{\partial T}{\partial \dot{z}_{1}}\right)-\frac{\partial T}{\partial z_{1}}=Q_{z_{1}} .
\end{aligned}
$$

Taking into account (F.4)-(F.5), (F.6) in the noninertial reference frame $\mathscr{B}$ will finally take the following form:

$$
\begin{gathered}
m\left(\frac{d^{2} x_{1}}{d t^{2}}\right)-m\left(\frac{d \varphi_{e}}{d t}\right)^{2} x_{1}-m\left(\frac{d^{2} \varphi_{e}}{d t^{2}}\right)\left(R+y_{1}\right) \\
-2 m\left(\frac{d \varphi_{e}}{d t}\right)\left(\frac{d y_{1}}{d t}\right)=-N\left(\frac{x_{1}}{l}\right) ; \\
m\left(\frac{d^{2} y_{1}}{d t^{2}}\right)-m\left(\frac{d \varphi_{e}}{d t}\right)^{2}\left(R+y_{1}\right)+m\left(\frac{d^{2} \varphi_{e}}{d t^{2}}\right) x_{1} \\
+2 m\left(\frac{d \varphi_{e}}{d t}\right)\left(\frac{d x_{1}}{d t}\right)=-N\left(\frac{y_{1}}{l}\right) ; \\
m\left(\frac{d^{2} z_{1}}{d t^{2}}\right)=-m g+N\left(\frac{l-z_{1}}{l}\right) .
\end{gathered}
$$

The derived ODE system (F.7) coincides with the system (F.3), which confirms the accuracy and correctness of the fully nonlinear equations (F.3) in relative Cartesian coordinates for payload $M$ swaying motion in the noninertial reference frame $\mathscr{B}$.

\section{G. Uniform Crane Boom Rotation Assumption: The Introduction of the Noninertial Reference Frame $\mathscr{F}$}

We now address the case of uniform crane boom slewing. We assume that the crane boom $\mathrm{BO}_{2}$ rotates with the constant 
angular velocity $\omega_{e}$ around vertical axis $\mathrm{DO}_{2}$; that is, that the noninertial reference frame $\mathscr{B}$ uniformly rotates around the unit vector $\widehat{\mathbf{e}}_{3}$ of the inertial reference frame $\mathscr{E}$. Such case takes place when the right-hand side of (E.5) is zero, that is, for the steady state of crane boom rotation with

$$
\frac{d^{2} \varphi_{e}}{d t^{2}}=0 ; \quad \frac{d \varphi_{e}}{d t}=\omega_{e}=\text { const. }
$$

So, taking into account (G.1), the third terms, containing $d^{2} \varphi_{e} / d t^{2}$, vanish in the 1 st and 2 nd equations of system (F.7). The second terms of (F.7) are linearly coordinate-dependent on $x_{1}, y_{1}$, and the forth terms of (F.7) are linearly velocitydependent on $d x_{1} / d t$ and $d y_{1} / d t$. So in the noninertial reference frame $\mathscr{B}$ we have

$$
\begin{aligned}
& m\left(\frac{d^{2} x_{1}}{d t^{2}}\right)-m\left(\frac{d \varphi_{e}}{d t}\right)^{2} x_{1} \\
& \quad-2 m\left(\frac{d \varphi_{e}}{d t}\right)\left(\frac{d y_{1}}{d t}\right)=-N\left(\frac{x_{1}}{l}\right) ; \\
& m\left(\frac{d^{2} y_{1}}{d t^{2}}\right)-m\left(\frac{d \varphi_{e}}{d t}\right)^{2}\left(R+y_{1}\right) \\
& +2 m\left(\frac{d \varphi_{e}}{d t}\right)\left(\frac{d x_{1}}{d t}\right)=-N\left(\frac{y_{1}}{l}\right) ; \\
& m\left(\frac{d^{2} z_{1}}{d t^{2}}\right)=-m g+N\left(\frac{l-z_{1}}{l}\right) .
\end{aligned}
$$

The nonlinear system (G.2) has been presented in the noninertial reference frame $\mathscr{B}$ for the case of uniform crane boom slewing.

For further physical analysis of the swaying problem and for ease of building the analytical solution we introduce the noninertial reference frame $\mathscr{F}$. The origin of the noninertial reference frame $\mathscr{F}$ we connect with the so-called point $A_{\text {dyn }}$ of dynamic equilibrium for load $M$, where the $y$ distance between the noninertial references frames $\mathscr{B}$ and $\mathscr{F}$ we define through the numerical solution of the following relative equilibrium transcendental equation:

$$
m g l \cdot \sin \left(\alpha_{1 \mathrm{dyn}}\right)=m \omega_{e}^{2}\left(R+l \cdot \sin \left(\alpha_{1 \mathrm{dyn}}\right)\right) \cdot l \cdot \cos \left(\alpha_{1 \mathrm{dyn}}\right)
$$

This equation (G.3) has been derived as the momentum sum for the gravitational force $\mathbf{m g}$ and the normal inertial force $\Phi_{\mathbf{e}}{ }^{\mathbf{n}}$ about point $B$, that is, $M_{B}(\mathbf{m g})=M_{B}\left(\Phi_{\mathbf{e}}{ }^{\mathbf{n}}\right)$.

Taking into account the numerically derived angle $\alpha_{1 \text { dyn }}$ from (G.3) we find the value of the horizontal distance $y_{\text {dyn }}$ between points $A_{\text {st }}$ and $A_{\text {dyn }}$ (Figures 8-10) according to the following equation:

$$
y_{\mathrm{dyn}}=A_{\mathrm{st}} A_{\mathrm{dyn}}=l \sin \left(\alpha_{1 \mathrm{dyn}}\right) .
$$

Relative coordinates $x, y$, and $z$ in noninertial reference frame $\mathscr{F}$ have been connected with the relative coordinates $x_{1}, y_{1}$, and $z_{1}$ in noninertial reference frame $\mathscr{B}$ according to the following equations (Figures 8-10):

$$
\begin{aligned}
& x_{1}=x ; \\
& y_{1}=y_{\mathrm{dyn}}+y ; \\
& z_{1}=\Delta+z .
\end{aligned}
$$

With (G.3)-(G.5) the nonlinear system (G.2) in the noninertial reference frame $\mathscr{F}$ takes the following form (Figures 8-10):

$$
\begin{gathered}
m\left(\frac{d^{2} x}{d t^{2}}\right)-m\left(\frac{d \varphi_{e}}{d t}\right)^{2} x \\
-2 m\left(\frac{d \varphi_{e}}{d t}\right)\left(\frac{d y}{d t}\right)=-N\left(\frac{x}{l}\right) ; \\
m\left(\frac{d^{2} y}{d t^{2}}\right)-m\left(\frac{d \varphi_{e}}{d t}\right)^{2}\left(R+y_{\mathrm{dyn}}+y\right) \\
+2 m\left(\frac{d \varphi_{e}}{d t}\right)\left(\frac{d x}{d t}\right)=-N\left(\frac{y_{\mathrm{dyn}}+y}{l}\right) ; \\
m\left(\frac{d^{2} z}{d t^{2}}\right)=-m g+N\left(\frac{l-\Delta-z}{l}\right) .
\end{gathered}
$$

\section{H. The Small Swaying Angle $\alpha_{1}$ Assumption}

We now address the case of the small swaying angle $\alpha_{1}$. In the case of small swaying angle $\alpha_{1}$ the system (C.3) defines $x_{1}$ and $y_{1}$ as the small variables, and $z_{1}=0$. Having $z_{1}=0$ we conclude that $\Delta=0$ and all time derivatives are zero, that is, the vertical load velocity $d z_{1} / d t=0$ and the vertical payload acceleration $d^{2} z_{1} / d t^{2}=0$.

Thus, the 3 rd equation of system (F.7) yields that the cable reaction force $\mathbf{N}$ approximately coincides the gravitational force $\mathbf{m g}$; that is, $\mathbf{N} \approx \mathbf{m g}$. As a result the system (G.2), containing three ODEs, transforms into a linearized system with two independent equations for the relative Cartesian coordinates $x_{1}$ and $y_{1}$ in the noninertial reference frame $\mathscr{B}$. We then cancel the mass $m$ of load $M$ from the system of (G.6). Consider

$$
\begin{gathered}
\frac{d^{2} x_{1}}{d t^{2}}-\left(\frac{d \varphi_{e}}{d t}\right)^{2} x_{1}+g\left(\frac{x_{1}}{l}\right)-2\left(\frac{d \varphi_{e}}{d t}\right)\left(\frac{d y_{1}}{d t}\right)=0 \\
\frac{d^{2} y_{1}}{d t^{2}}-\left(\frac{d \varphi_{e}}{d t}\right)^{2}\left(R+y_{1}\right)+g\left(\frac{y_{1}}{l}\right)+2\left(\frac{d \varphi_{e}}{d t}\right)\left(\frac{d x_{1}}{d t}\right)=0 .
\end{gathered}
$$

So we have derived the system (H.1) of differential equations for relative motion of load $M$ on cable $B M$ with a movable suspension center $B$, which is attached to the crane boom $\mathrm{BO}_{2}$ in the noninertial reference frame $\mathscr{B}$ We then transfer the origin of coordinate system $O x y z$ (Figures 8-10) to the point $A_{\mathrm{dyn}}$ of dynamic equilibrium for load $M$; that is, 
we make the transition from the noninertial reference frame $\mathscr{B}$ to the noninertial reference frame $\mathscr{F}$ and simplify (G.5) to

$$
\begin{aligned}
& x_{1}=x ; \\
& y_{1}=y_{\text {dyn }}+y ; \\
& z_{1}=z .
\end{aligned}
$$

The second equation of system (H.1) defines the amount of dynamic deflection, that is, the $y$-distance between the noninertial reference frames $\mathscr{B}$ and $\mathscr{F}$ as

$$
y_{\mathrm{dyn}}=A_{\mathrm{st}} A_{\mathrm{dyn}}=\frac{\omega_{e}^{2} R l}{g-\omega_{e}^{2} l}=-\frac{\omega_{e}^{2} R}{\left(\omega_{e}^{2}-(g / l)\right)},
$$

where $\omega_{e}=d \varphi_{e} / d t$.

Then with the introduction of (H.2) and (H.3) into (H.1) we have the normal system of two linear homogeneous differential equations of second order for relative motion of the load $M$ in the noninertial reference frame $\mathscr{F}$ :

$$
\begin{aligned}
& \frac{d^{2} x}{d t^{2}}-\left(\left(\frac{d \varphi_{e}}{d t}\right)^{2}-\left(\frac{g}{l}\right)\right) x-2\left(\frac{d \varphi_{e}}{d t}\right)\left(\frac{d y}{d t}\right)=0 \\
& \frac{d^{2} y}{d t^{2}}-\left(\left(\frac{d \varphi_{e}}{d t}\right)^{2}-\left(\frac{g}{l}\right)\right) y+2\left(\frac{d \varphi_{e}}{d t}\right)\left(\frac{d x}{d t}\right)=0 .
\end{aligned}
$$

The same system in the noninertial reference frame $\mathscr{F}$ can be derived from (G.6) for the case of the small swaying angle $\alpha_{1}$. Taking into account that $\omega_{e}=d \varphi_{e} / d t=$ const we will write (H.4) as

$$
\begin{aligned}
& \frac{d^{2} x}{d t^{2}}-\left(\omega_{e}^{2}-\left(\frac{g}{l}\right)\right) x-2 \omega_{e}\left(\frac{d y}{d t}\right)=0 \\
& \frac{d^{2} y}{d t^{2}}-\left(\omega_{e}^{2}-\left(\frac{g}{l}\right)\right) y+2 \omega_{e}\left(\frac{d x}{d t}\right)=0 .
\end{aligned}
$$

Taking into account (C.6), the absolute coordinates $x_{2}, y_{2}$, and $z_{2}$ in inertial reference frame $\mathscr{E}$, which depend upon the relative coordinates $x, y$, and $z$ in the noninertial reference frame $\mathscr{F}$ during the swaying of load $M$, will be defined according to the following equations (Figures 8-10):

$$
\begin{aligned}
& x_{2}=\left(R+y_{\mathrm{dyn}}+y\right) \cos \left(\varphi_{e}\right)+x \sin \left(\varphi_{e}\right) ; \\
& y_{2}=\left(R+y_{\mathrm{dyn}}+y\right) \sin \left(\varphi_{e}\right)-x \cos \left(\varphi_{e}\right) ; \\
& z_{2}=z .
\end{aligned}
$$

So the linearized ODE system (H.4)-(H.5) has been derived for the case of uniform crane boom slewing (G.1) and the small swaying angle $\alpha_{1}$ (H.2).

It is important to note that the left-hand sides of payload motion equations in linearized problem (H.4)-(H.5) for the noninertial reference frame $\mathscr{F}$ may be derived with an introduction of Lagrange equations (F.6). However the discussion of (H.4)-(H.5) is more suitable and informative with an introduction of dynamic Coriolis theorem (F.1)-(F.2). The first terms $d^{2} x / d t^{2} ; d^{2} y / d t^{2}$ in (H.4)-(H.5) define the vector
$\mathbf{a}_{M / \mathscr{F}}=\mathbf{a}_{\mathbf{r}}$ (B.4) for the relative acceleration of load $M$ in the noninertial reference frame $\mathscr{F}$. The straight-line terms $\left(\omega_{e}^{2}-(g / l)\right) x$ and $\left(\omega_{e}^{2}-(g / l)\right) y$ in (H.4)-(H.5) are linear proportional to the relative payload coordinates in the noninertial reference frame $\mathscr{F}$. These straight-line terms have been determined by the contribution of the normal or centripetal acceleration $\boldsymbol{\omega}_{\mathscr{B} / \mathscr{E}} \times\left(\boldsymbol{\omega}_{\mathscr{B} / \mathscr{E}} \times \mathbf{r}_{E / M}\right)=\mathbf{a}_{\mathbf{e}}{ }^{\mathbf{n}}$ of transportation for payload $M$ and by appearance of corresponding D'Alembert centrifugal inertia force $\boldsymbol{\Phi}_{\mathbf{e}}{ }^{\mathbf{n}}=(-m) \mathbf{a}_{\mathbf{e}}{ }^{\mathbf{n}}$ due to crane boom transport rotation in the noninertial reference frame $\mathscr{B}$. The third terms $-2 \omega_{e}(d y / d t)$ and $2 \omega_{e}(d x / d t)$ in (H.4)-(H.5) have been defined by the compound or Coriolis acceleration $2 \boldsymbol{\omega}_{\mathscr{B} / \mathscr{C}} \times \mathbf{V}_{M / \mathscr{B}}=\mathbf{a}_{\text {cor }}$ of payload $M$ in the noninertial reference frame $\mathscr{F}$. The rectangular Cartesian projections of Coriolis inertia force in the noninertial reference frame $\mathscr{F}$ are defined by formula (D.5).

\section{Analytical Solution of the Linearized System}

After generation of the determinant of natural frequencies matrix for the system (H.4)-(H.5) we will write the following characteristic biquadratic equation of fourth order for system (H.4)-(H.5) in the form:

$$
\lambda^{4}+2\left(\left(\frac{g}{l}\right)+\omega_{e}^{2}\right) \lambda^{2}+\left(\left(\frac{g}{l}\right)-\omega_{e}^{2}\right)^{2}=0
$$

Using (I.1) we adjust the roots $\lambda_{1}$ and $\lambda_{2}$ of the secular equation:

$$
\begin{array}{cl}
\lambda_{1}= \pm \nu_{1} i ; & \lambda_{2}= \pm v_{2} i, \\
v_{1}=k+\omega_{e} ; & v_{2}=k-\omega_{e} ; \\
k=\sqrt{\frac{g}{l} ;} & \omega_{e} \neq k .
\end{array}
$$

Taking into account (I.2), the law of relative load $M$ motion in the noninertial reference frame $\mathscr{F}$ takes the following form:

$$
\begin{aligned}
x(t)= & C_{1} \sin \left(\nu_{1} t\right)+C_{2} \cos \left(\nu_{1} t\right) \\
& +C_{3} \sin \left(\nu_{2} t\right)+C_{4} \cos \left(\nu_{2} t\right) ; \\
y(t)= & C_{1} \cos \left(\nu_{1} t\right)-C_{2} \sin \left(\nu_{1} t\right) \\
& -C_{3} \cos \left(\nu_{2} t\right)+C_{4} \sin \left(\nu_{2} t\right) .
\end{aligned}
$$

Time derivatives of (I.3) yield the following projections of relative payload $M$ velocity in the noninertial reference frame $\mathscr{F}$ :

$$
\begin{aligned}
\frac{d(x(t))}{d t}= & +C_{1} \nu_{1} \cos \left(\nu_{1} t\right)-C_{2} \nu_{1} \sin \left(\nu_{1} t\right) \\
& +C_{3} \nu_{2} \cos \left(\nu_{2} t\right)-C_{4} \nu_{2} \sin \left(\nu_{2} t\right) \\
\frac{d(y(t))}{d t}= & -C_{1} \nu_{1} \sin \left(\nu_{1} t\right)-C_{2} \nu_{1} \cos \left(\nu_{1} t\right) \\
& +C_{3} \nu_{2} \sin \left(\nu_{2} t\right)+C_{4} \nu_{2} \cos \left(\nu_{2} t\right)
\end{aligned}
$$


In order to determine the arbitrary constants of integration $C_{1}, \ldots, C_{4}$ in (I.3)-(I.4) we define the initial conditions of the problem according to Figures 8-10 and Figure 11.

In the initial time $t=0$ load $M$ has the vertical $B A_{\text {st }}$ inline position (Figures 8-10); that is, the load $M$ initial position coincides with the static equilibrium position $A_{\text {st }}$ for the load $M$ on the cable $M B$. This means that the initial coordinates of payload $M$ are as follows:

point $A_{\text {st }}(R, 0,0)$ in the reference frame $\mathscr{E}$;

point $A_{\text {st }}(0,0,0)$ in the reference frame $\mathscr{B}$;

point $A_{\text {st }}\left(0,-y_{\text {dyn }}, 0\right)$ in the reference frame $\mathscr{F}$.

At time $t=0$ the load $M$ has zero absolute velocity in the inertial reference frame $\mathscr{E}$; that is, $\mathbf{V}_{M / \mathscr{E}}(0)=\mathbf{0}$.

In order to determine the load $M$ relative velocity in the noninertial reference frame $\mathscr{F}$, which is equal to the load $M$ relative velocity in the noninertial reference frame $\mathscr{B}$, we address the velocity addition theorem (A.12) and formula (A.14):

$$
\mathbf{0}=\mathbf{V}_{M / \mathscr{B}}(0)+\boldsymbol{\omega}_{\mathscr{B} / \mathscr{E}}(0) \times \mathbf{r}_{\mathrm{O}_{2} / M}(0)
$$

The vector equation (I.8) determines the initial relative velocity $\mathbf{V}_{M / \mathscr{B}}(0)$ of the load $M$ in the inertial reference frame $\mathscr{E}$ as

$$
\begin{aligned}
\mathbf{V}_{M / \mathscr{B}}(0) & =\mathbf{V}_{M / \mathscr{F}}(0)=-\boldsymbol{\omega}_{\mathscr{B} / \mathscr{E}}(0) \times \mathbf{r}_{\mathrm{O}_{2} / M}(0) \\
& =-\boldsymbol{\omega}_{\mathscr{B} / \mathscr{E}}(0) \times\left\|\mathbf{r}_{\mathrm{O}_{2} / M}(0)\right\| \widehat{\mathbf{e}}_{2}
\end{aligned}
$$

The direction of the vector for the initial relative velocity $\mathbf{V}_{M / \mathscr{B}}(0)$ is directed opposite to the unit vector $\widehat{\mathbf{e}}_{2}$ in the inertial reference frame $\mathscr{E}$; that is, $\mathbf{V}_{M / \mathscr{B}}(0) \uparrow \downarrow \widehat{\mathbf{e}}_{2}$. The initial direction of the vector $\mathbf{V}_{M / \mathscr{B}}(0)$ is invariant in all reference systems $\mathscr{E}, \mathscr{B}$, and $\mathscr{F}$. So in the noninertial reference frame $\mathscr{B}$ we have $\mathbf{V}_{M / B}(0) \uparrow \uparrow \widetilde{\mathbf{e}}_{1}$ :

$$
\mathbf{V}_{M / \mathscr{B}}(0)=\mathbf{V}_{M / \mathscr{F}}(0)=+\boldsymbol{\omega}_{\mathscr{B} / \mathscr{C}}(0) \times\left\|\mathbf{r}_{O_{2} / M}(0)\right\| \widetilde{\mathbf{e}}_{1}
$$

Taking into account that $\left\|\boldsymbol{\omega}_{\mathscr{B} / \mathscr{E}}(0)\right\|=\omega_{e}$ and $\left\|\mathbf{r}_{\mathrm{O}_{2} / M}(0)\right\|=R$ in (I.10), we have the following scalar equation in the noninertial reference frames $\mathscr{B}$ and $\mathscr{F}$ :

$$
\mathbf{V}_{M / \mathscr{B}}(0)=\mathbf{V}_{M / \mathscr{F}}(0)=\frac{d x}{d t}(0)=\omega_{e} R
$$

Thus, with an introduction of (I.5)-(I.11), the initial conditions for ODE system (H.4)-(H.5) in the noninertial reference frame $\mathscr{F}$ are as follows:

$$
\begin{gathered}
x(0)=0 ; \quad \frac{d x}{d t}(0)=\omega_{e} R ; \\
y(0)=-y_{\text {dyn }} ; \quad \frac{d y}{d t}(0)=0 .
\end{gathered}
$$

The substitution of (I.12) into (I.3) and (I.4) yields the following system for derivation the analytical expressions for the arbitrary constants of integration $C_{1}, \ldots, C_{4}$ :

$$
\begin{gathered}
0=C_{2}+C_{4} ; \\
V_{B}=C_{1} \nu_{1}+C_{2} \nu_{2} ; \\
-y_{\text {dyn }}=C_{1}-C_{3} ; \\
0=-C_{2} \nu_{1}+C_{4} \nu_{2} .
\end{gathered}
$$

The algebraic expressions for the arbitrary constants of integration $C_{1}, \ldots, C_{4}$ can be derived as the following solution of the system (I.13):

$$
\begin{aligned}
& C_{1}=\frac{V_{B}-y_{\text {dyn }} \nu_{2}}{\nu_{1}+\nu_{2}} ; \quad C_{2}=0 ; \\
& C_{3}=\frac{V_{B}+y_{\text {dyn }} \nu_{1}}{\nu_{1}+v_{2}} ; \quad C_{4}=0 ; \\
& \text { or } \quad C_{1}=\frac{V_{B}-y_{\text {dyn }}\left(k-\omega_{e}\right)}{2 k} ; \quad C_{2}=0 ; \\
& C_{3}=\frac{V_{B}+y_{\text {dyn }}\left(k+\omega_{e}\right)}{2 k} ; \quad C_{4}=0 .
\end{aligned}
$$

So, the relative motion equations for pendulum $M$ swaying take the final form as stated below:

$$
\begin{aligned}
x(t)= & \left(\frac{V_{B}-y_{\mathrm{dyn}}\left(k-\omega_{e}\right)}{2 k}\right) \sin \left(\nu_{1} t\right) \\
& +\left(\frac{V_{B}+y_{\mathrm{dyn}}\left(k+\omega_{e}\right)}{2 k}\right) \sin \left(\nu_{2} t\right) ; \\
y(t)= & \left(\frac{V_{B}-y_{\mathrm{dyn}}\left(k-\omega_{e}\right)}{2 k}\right) \cos \left(\nu_{1} t\right) \\
& -\left(\frac{V_{B}+y_{\mathrm{dyn}}\left(k+\omega_{e}\right)}{2 k}\right) \cos \left(\nu_{2} t\right) .
\end{aligned}
$$

The obtained analytical equations (I.15), derived for the linearized model (H.4)-(H.5), determine the shape of the relative payload $M$ swaying trajectory as is shown in Figure 12. Numerical computations in Figure 12 have been carried out for the following values of mechanical system parameters: $R=0.492 \mathrm{~m} ; g=9.81 \mathrm{~m} / \mathrm{s}^{2} ; l=0.825 \mathrm{~m} ; k=(g / l)^{0.5} \approx$ $3.448 \mathrm{rad} / \mathrm{s} ; T=30 \mathrm{~s} ; \omega_{e}=2 \pi / T \approx 0.209 \mathrm{rad} / \mathrm{s} ; \varphi_{e}=180^{\circ}$; $\alpha_{1 \mathrm{dyn}}=0.00221 \mathrm{rad} ; V_{B}=0.103 \mathrm{~m} / \mathrm{s} ; y_{\mathrm{dyn}}=0.00182 \mathrm{~m}$; $v_{1}=k+\omega_{e}=3.6578 \mathrm{rad} / \mathrm{s} ; \nu_{2}=k-\omega_{e}=3.2388 \mathrm{rad} / \mathrm{s}$; $C_{1}=0.01408 \mathrm{~m}$; and $C_{3}=0.01591 \mathrm{~m}$ (Figure 12). The above mentioned numerical values of analytical relations (I.15) subject to the initial conditions (I.5)-(I.12) define a theoretically computed law of relative motion for load $M$. After the elimination of variable $t$ from the system of (I.15) we will have the relative trajectory for load $M$ (Figure 12). The system (H.6) and the computational relative trajectories for load $M$ swaying in Figure 12 show that there is the contrarotation of the noninertial reference frames $\mathscr{B}$ and $\mathscr{F}$ to crane boom $\mathrm{BO}_{2}$ slewing. 


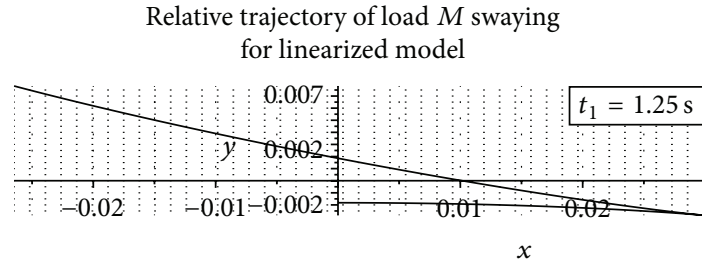

$y(x): l=0.825 \mathrm{~m} ; k \approx 3.448 \mathrm{rad} / \mathrm{s} ; \omega_{e} \approx 0.209 \mathrm{rad} / \mathrm{s}$

(a)

Relative trajectory of load $M$ swaying for linearized model

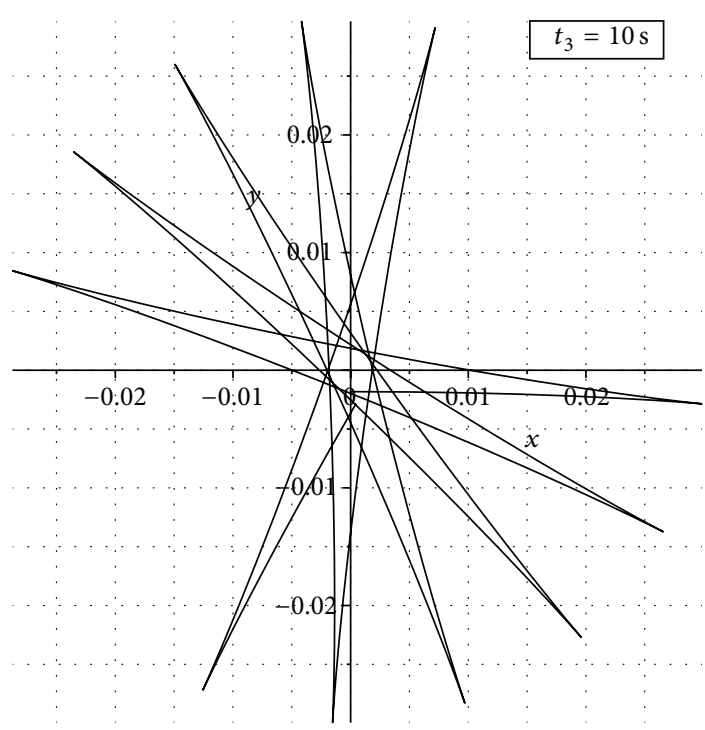

$-y(x): l=0.825 \mathrm{~m} ; k \approx 3.448 \mathrm{rad} / \mathrm{s} ; \omega_{e} \approx 0.209 \mathrm{rad} / \mathrm{s}$

(c)

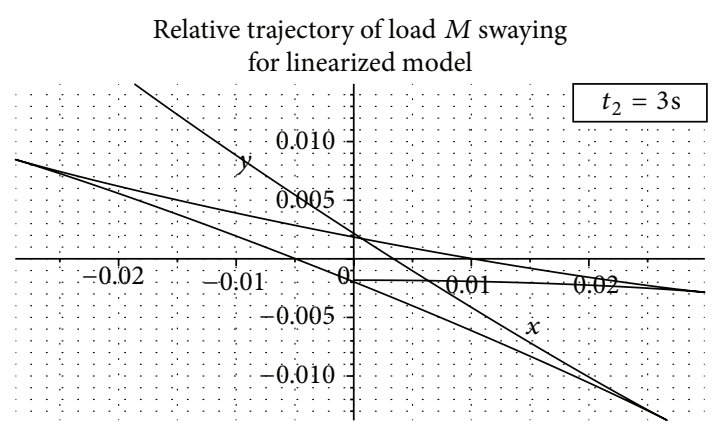

$y(x): l=0.825 \mathrm{~m} ; k \approx 3.448 \mathrm{rad} / \mathrm{s} ; \omega_{e} \approx 0.209 \mathrm{rad} / \mathrm{s}$

(b)

Relative trajectory of load $M$ swaying for linearized model

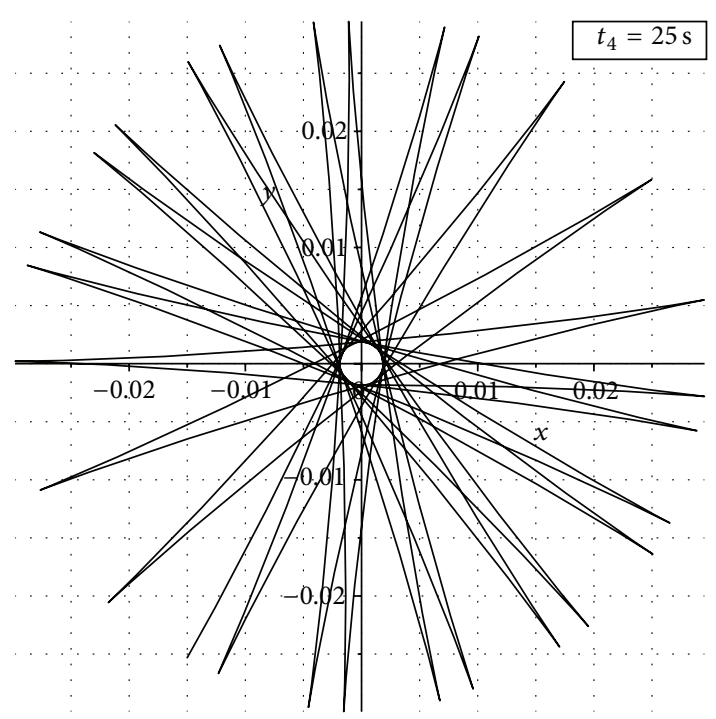

$y(x): l=0.825 \mathrm{~m} ; k \approx 3.448 \mathrm{rad} / \mathrm{s} ; \omega_{e} \approx 0.209 \mathrm{rad} / \mathrm{s}$

(d)

FIGURE 12: The relative trajectories for load $M$ swaying on the cable during uniform rotational motion of the crane boom $\mathrm{BO}_{2}$ for the moments of times $t_{1}=1.25 \mathrm{~s}(\mathrm{a}), t_{2}=3 \mathrm{~s}(\mathrm{~b}), t_{3}=10 \mathrm{~s}$, (c) and $t_{4}=25 \mathrm{~s}(\mathrm{~d})$, derived with linearized model introduction.

\section{J. Velocity Kinematics Analysis in \\ Relative Spherical Coordinates of the Noninertial Reference Frame $\mathscr{B}$ : The Introduction of the Noninertial Reference Frames $\mathscr{C}, \mathscr{D}$, and $\mathscr{G}$}

The fully nonlinear equations (F.7) in relative Cartesian coordinates of the noninertial reference frame $\mathscr{B}$ have the unknown function $N=N(t)$, that is, undetermined Lagrangian coefficient. The reaction force $\mathbf{N}$ of the cable $B M$ in Figures 8-10 and Figure 13 is the nonconservative force.

So for derivation of solvable nonlinear equations we should remove the cable tension force $\mathbf{N}$ from the payload $M$ motion equations with the introduction of Newton's second law and the natural noninertial comoving reference frames $\mathscr{C}, \mathscr{D}$, and $\mathscr{G}$, which correspond to the spherical angular coordinates $\left(\alpha_{1}, \alpha_{2}\right.$, and $\left.\varphi_{e}\right)$ and moving together with payload $M$ (Figure 13). The advantage of the introduction of the natural noninertial comoving reference frames $\mathscr{C}, \mathscr{D}$, and $\mathscr{G}$ is grounded on the orthogonality of the reaction force $\mathbf{N}$ to the unit vectors $\mathbf{e}_{\tau \alpha_{1}}$ and $\mathbf{e}_{\tau \alpha_{2}}$, that is, on the fact that $\mathbf{N} \perp \mathbf{e}_{\tau \alpha_{1}}$ and $\mathbf{N} \perp \mathbf{e}_{\tau \alpha_{2}}$.

The vector equation (A.12) for velocity addition will take the form

$$
\mathbf{V}_{M / \mathscr{C}}=\mathbf{V}_{M / \mathscr{C}}+\mathbf{V}_{M / \mathscr{D}}+\boldsymbol{\omega}_{\mathscr{B} / \mathscr{C}} \times \mathbf{r}_{\mathrm{O}_{2} / M}
$$

where $\mathbf{V}_{M / \mathscr{C}}=\mathbf{V}_{\alpha_{1}}$ and $\mathbf{V}_{M / \mathscr{D}}=\mathbf{V}_{\alpha_{2}}$ are the relative payload velocities in the natural noninertial comoving reference frames $\mathscr{C}$ and $\mathscr{D}$.

In Figure 13 and by using (J.1) we have that the natural components of payload $M$ velocity in the natural noninertial 


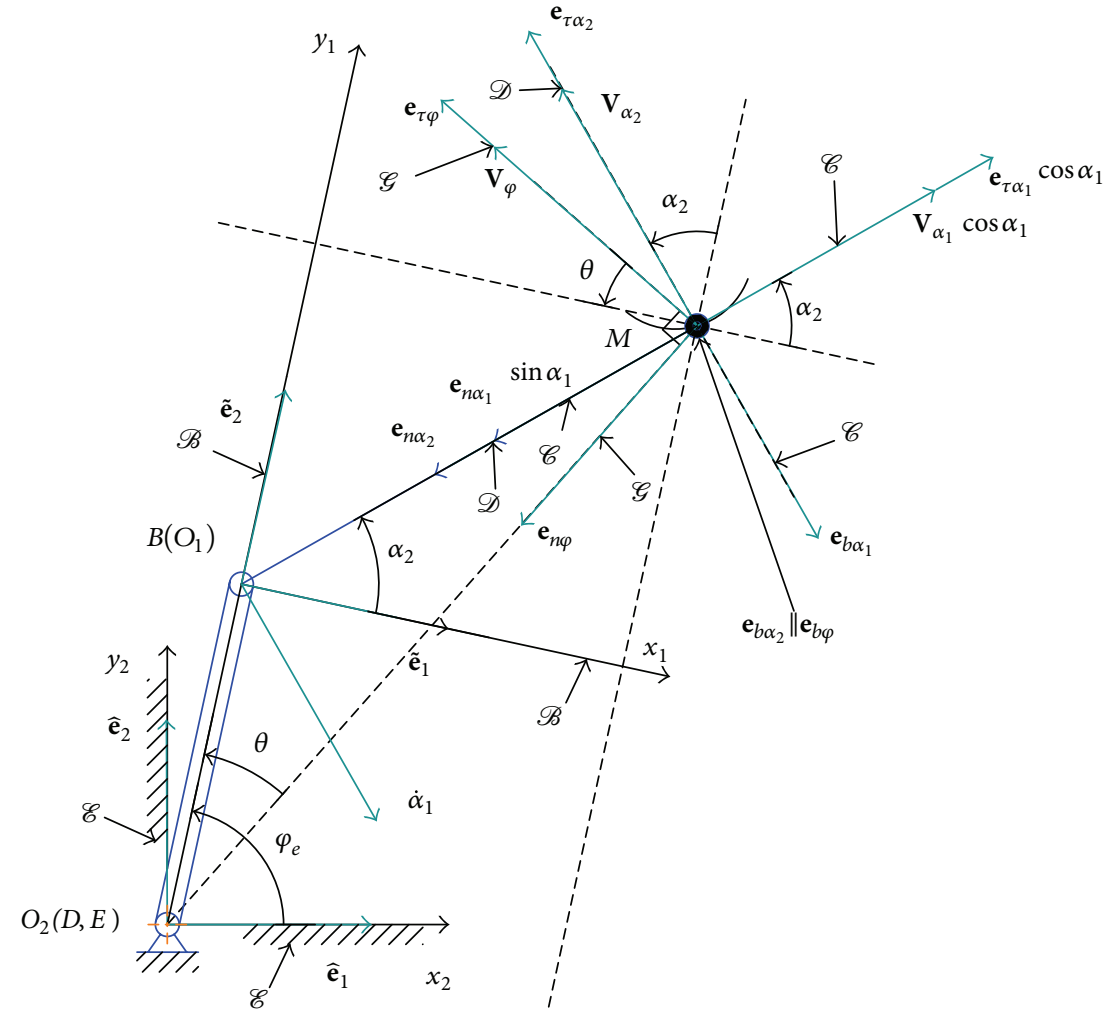

FIGURE 13: Swaying scheme for velocity kinematics analysis in spherical coordinates of load $M$ at the cable $M B$, which is fixedly attached in the point $B$ to the crane boom $B O_{2}$, in the horizontal plane $\left(x_{1} y_{1}\right)$ for nonlinear model derivation in relative spherical coordinates.

comoving reference frames $\mathscr{C}, \mathscr{D}$, and $\mathscr{G}$ have the following values:

$$
\begin{aligned}
& V_{\alpha_{1}}=l \frac{d \alpha_{1}}{d t} ; \\
& V_{\alpha_{2}}=l \sin \left(\alpha_{1}\right) \frac{d \alpha_{2}}{d t} ; \\
& V_{\varphi}=O_{2} M \frac{d \varphi_{e}}{d t} .
\end{aligned}
$$

\section{K. Acceleration Kinematics \\ Analysis in Relative Spherical Coordinates in the Noninertial Reference Frames $\mathscr{C}, \mathscr{D}$, and $\mathscr{G}$}

The vector equation (B.1) for acceleration addition will take the following form (Figure 14):

$$
\begin{aligned}
\mathbf{a}_{M / \mathscr{C}}= & \mathbf{a}_{M / \mathscr{C}}+\mathbf{a}_{M / \mathscr{D}}+2 \boldsymbol{\omega}_{\mathscr{C} / \mathscr{E}} \times \mathbf{v}_{M / \mathscr{C}} \\
& +2 \boldsymbol{\omega}_{\mathscr{D} / \mathscr{E}} \times \mathbf{v}_{M / \mathscr{D}} \\
& +\boldsymbol{\omega}_{\mathscr{D} / \mathscr{C}} \times\left(\boldsymbol{\omega}_{\mathscr{D} / \mathscr{E}} \times \mathbf{r}_{\mathrm{O}_{2} / M}\right)+\boldsymbol{\alpha}_{\mathscr{D} / \mathscr{C}} \times \mathbf{r}_{\mathrm{O}_{2} / M},
\end{aligned}
$$

where $\mathbf{a}_{\text {cor }}\left(\mathbf{V}_{M / \mathscr{C}}\right)=\mathbf{a}_{\text {cor }}\left(\mathbf{V}_{\alpha_{1}}\right)=2 \boldsymbol{\omega}_{\mathscr{C} / \mathscr{E}} \times \mathbf{V}_{M / \mathscr{C}}=2 \boldsymbol{\omega}_{\mathscr{C} / \mathscr{E}} \times \mathbf{V}_{\alpha_{1}}$ and $\mathbf{a}_{\text {cor }}\left(\mathbf{V}_{M / \mathscr{D}}\right)=\mathbf{a}_{\text {cor }}\left(\mathbf{V}_{\alpha_{2}}\right)=2 \boldsymbol{\omega}_{\mathscr{D} / \mathscr{E}} \times \mathbf{V}_{M / \mathscr{D}}=2 \boldsymbol{\omega}_{\mathscr{D} / \mathscr{E}} \times \mathbf{V}_{\alpha_{2}}$ in Figure 14.
So formula (K.1) will take the following form:

$$
\begin{aligned}
\mathbf{a}_{M / \mathscr{C}}= & \mathbf{a}_{M / \mathscr{C}}+\mathbf{a}_{M / \mathscr{D}}+\mathbf{a}_{\text {cor }}\left(\mathbf{v}_{\alpha_{1}}\right)+\mathbf{a}_{\text {cor }}\left(\mathbf{V}_{\alpha_{2}}\right) \\
& +\boldsymbol{\omega}_{\mathscr{D} / \mathscr{E}} \times\left(\boldsymbol{\omega}_{\mathscr{D} / \mathscr{E}} \times \mathbf{r}_{\mathrm{O}_{2} / M}\right)+\boldsymbol{\alpha}_{\mathscr{D} / \mathscr{E}} \times \mathbf{r}_{\mathrm{O}_{2} / M} .
\end{aligned}
$$

The directions of vectors have been shown in Figures 13, 14 , and 15. The scalar values of vectors $\boldsymbol{\omega}_{\mathscr{C} / \mathscr{E}}$ and $\boldsymbol{\omega}_{\mathscr{D} / \mathscr{E}}$ are as follows:

$$
\begin{gathered}
\boldsymbol{\omega}_{\mathscr{C} / \mathscr{C}}=\frac{d \varphi_{e}}{d t}+\frac{d \alpha_{2}}{d t} ; \\
\boldsymbol{\omega}_{\mathscr{D} / \mathscr{E}}=\frac{d \varphi_{e}}{d t} .
\end{gathered}
$$

Taking into account (K.3), the magnitudes of Coriolis acceleration vectors in the natural noninertial comoving reference frames $\mathscr{C}, \mathscr{D}$, and $\mathscr{G}$ in Figure 14 have the following values:

$$
\begin{aligned}
& \mathbf{a}_{\text {cor }}\left(V_{\alpha_{1}}\right)=2 l\left(\frac{d \alpha_{1}}{d t}\right)\left(\frac{d \varphi_{e}}{d t}+\frac{d \alpha_{2}}{d t}\right) \cos \left(\alpha_{1}\right) ; \\
& \mathbf{a}_{\text {cor }}\left(V_{\alpha_{2}}\right)=2 l\left(\frac{d \alpha_{2}}{d t}\right)\left(\frac{d \varphi_{e}}{d t}\right) \sin \left(\alpha_{1}\right) .
\end{aligned}
$$




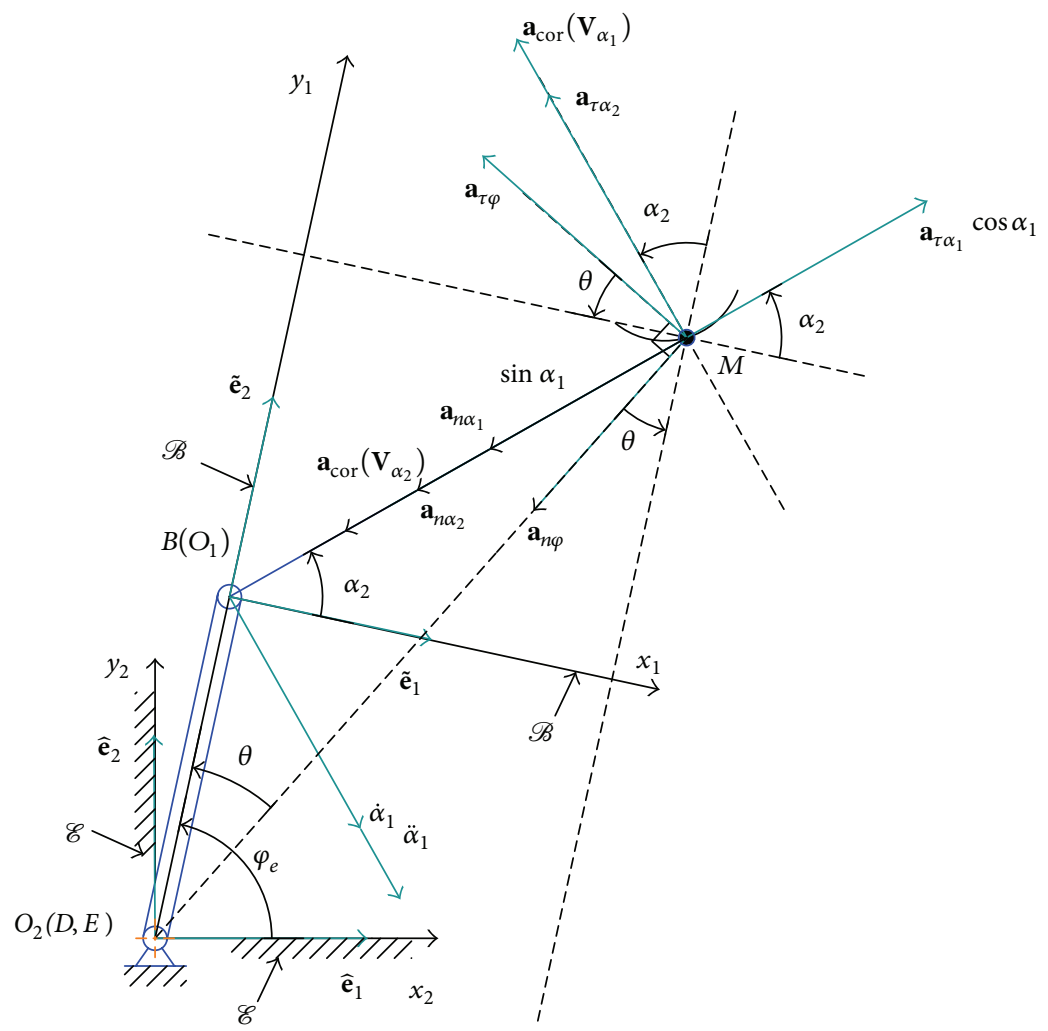

FIGURE 14: Swaying scheme for acceleration kinematics analysis in spherical coordinates of load $M$ at the cable $M B$, which is fixedly attached in the point $B$ to the crane boom $B O_{2}$, in the horizontal plane $\left(x_{1} y_{1}\right)$ for nonlinear model derivation in relative spherical coordinates.

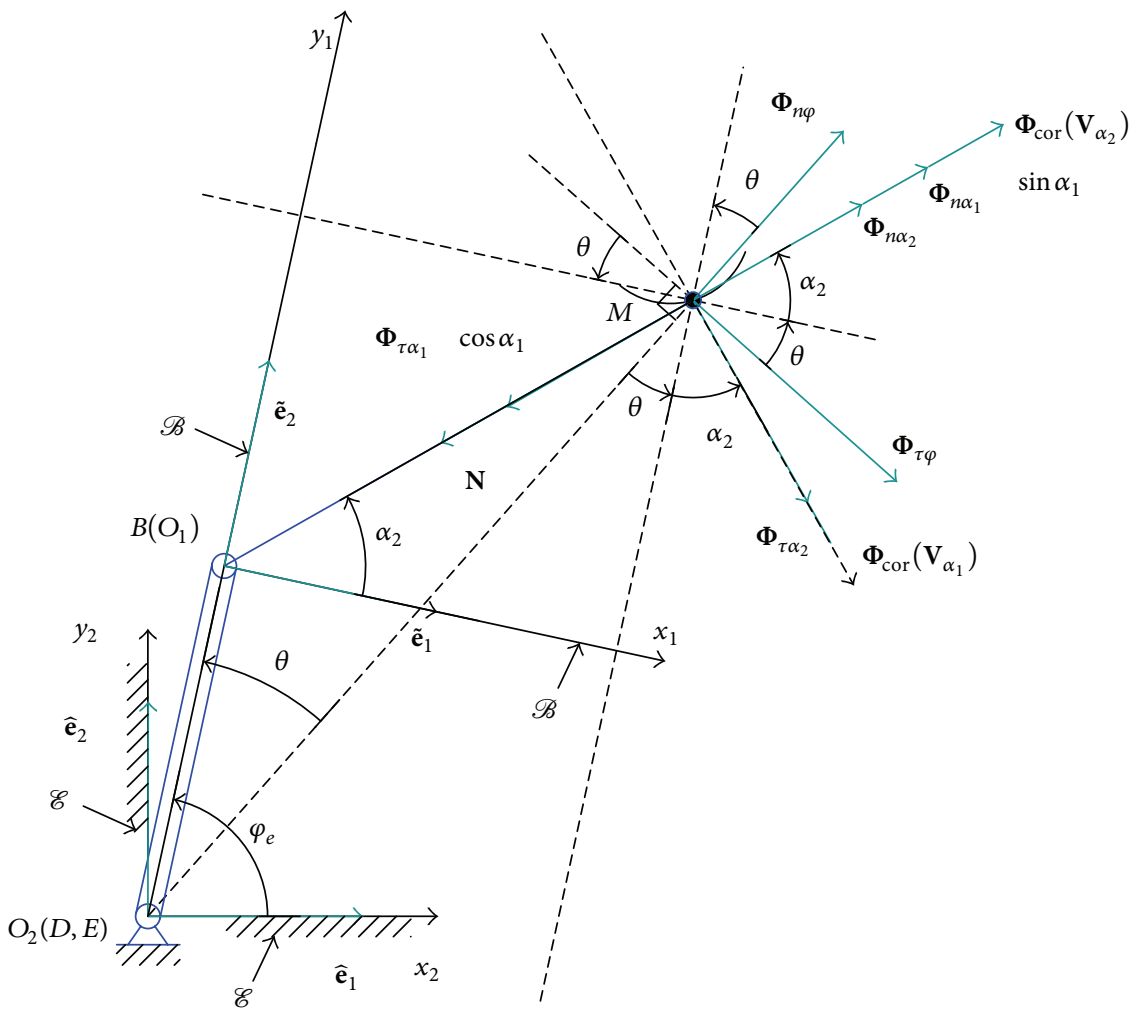

FIGURE 15: Swaying scheme for dynamic analysis in spherical coordinates of load $M$ at the cable $M B$, which is fixedly attached in the point $B$ to the crane boom $B \mathrm{O}_{2}$, in the horizontal plane $\left(x_{1} y_{1}\right)$ for nonlinear model derivation in relative spherical coordinates. 
The tangential accelerations in the natural noninertial comoving reference frames $\mathscr{C}, \mathscr{D}, \mathscr{G}$ have the values as stated below:

$$
\begin{aligned}
& a_{\tau \alpha_{1}}=\left(\frac{d^{2} \alpha_{1}}{d t^{2}}\right) l ; \\
& a_{\tau \alpha_{2}}=\left(\frac{d^{2} \alpha_{2}}{d t^{2}}\right) l \sin \left(\alpha_{1}\right) ; \\
& a_{\tau \varphi}=\left(\frac{d^{2} \varphi_{e}}{d t^{2}}\right)\left(O_{2} M\right) .
\end{aligned}
$$

The normal accelerations in the natural noninertial comoving reference frames $\mathscr{C}, \mathscr{D}, \mathscr{G}$ have the values as stated below:

$$
\begin{aligned}
& a_{n \alpha_{1}}=\left(\frac{d \alpha_{1}}{d t}\right)^{2} l ; \\
& a_{n \alpha_{2}}=\left(\frac{d \alpha_{2}}{d t}\right)^{2} l \sin \left(\alpha_{1}\right) ; \\
& a_{n \varphi}=\left(\frac{d \varphi_{e}}{d t}\right)^{2}\left(O_{2} M\right) .
\end{aligned}
$$

\section{Forces Imposed on the Payload in Relative Spherical Coordinates in the Noninertial Reference Frames $\mathscr{C}, \mathscr{D}$, and $\mathscr{G}$}

Among the forces, imposed on the payload $M$ in Figure 15, we have an active force of gravity $\mathbf{m g}$, the cable reaction force $\mathbf{N}$, the tangential inertial forces $\Phi_{\tau \alpha_{1}} ; \Phi_{\tau \alpha_{2}} ; \Phi_{\tau \varphi}$, the normal or centrifugal inertial forces $\Phi_{n \alpha_{1}} ; \Phi_{n \alpha_{2}} ; \Phi_{n \varphi}$, and the Coriolis inertial forces $\boldsymbol{\Phi}_{\text {cor }}\left(V_{\alpha_{1}}\right)$ and $\boldsymbol{\Phi}_{\text {cor }}\left(V_{\alpha_{2}}\right)$.

Taking into account formulae (K.3)-(K.7) we will express below all imposed forces in the noninertial reference frames $\mathscr{C}, \mathscr{D}, \mathscr{G}$ :

$$
\begin{aligned}
& \Phi_{\tau \alpha_{1}}=m a_{\tau \alpha_{1}}=m\left(\frac{d^{2} \alpha_{1}}{d t^{2}}\right) l ; \\
& \Phi_{\tau \alpha_{2}}=m a_{\tau \alpha_{2}}=m\left(\frac{d^{2} \alpha_{2}}{d t^{2}}\right) l \sin \left(\alpha_{1}\right) ; \\
& \Phi_{\tau \varphi}=m a_{\tau \varphi}=m\left(\frac{d^{2} \varphi_{e}}{d t^{2}}\right)\left\|O_{2} M\right\| ; \\
& \Phi_{n \alpha_{1}}=m a_{n \alpha_{1}}=m\left(\frac{d \alpha_{1}}{d t}\right)^{2} l ;
\end{aligned}
$$

$$
\begin{gathered}
\Phi_{n \alpha_{2}}=m a_{n \alpha_{2}}=m\left(\frac{d \alpha_{2}}{d t}\right)^{2} l \sin \left(\alpha_{1}\right) \\
\Phi_{n \varphi}=m a_{n \varphi}=m\left(\frac{d \varphi_{e}}{d t}\right)^{2}\left\|O_{2} M\right\| ; \\
\Phi_{\text {cor }}\left(V_{\alpha_{1}}\right)=m a_{\text {cor }}\left(V_{\alpha_{1}}\right) \\
=2 m L\left(\frac{d \alpha_{1}}{d t}\right)\left(\frac{d \varphi_{e}}{d t}+\frac{d \alpha_{2}}{d t}\right) \cos \left(\alpha_{1}\right) ; \\
\Phi_{\text {cor }}\left(V_{\alpha_{2}}\right)=m a_{\text {cor }}\left(V_{\alpha_{2}}\right) \\
=2 m L\left(\frac{d \alpha_{2}}{d t}\right)\left(\frac{d \varphi_{e}}{d t}\right) \sin \left(\alpha_{1}\right) .
\end{gathered}
$$

\section{Derivation of the Fully Nonlinear Equations in Relative Spherical Coordinates in the Noninertial Reference Frames $\mathscr{C}, \mathscr{D}$, and $\mathscr{G}$}

Taking into account derived expressions (L.1), the introduction of D'Alembert's principle in the noninertial reference frames $\mathscr{C}, \mathscr{D}, \mathscr{G}$ yields the following scalar equations in the projections to the axes $\mathbf{e}_{\tau \alpha_{1}}$ and $\mathbf{e}_{\tau \alpha_{2}}$ :

$$
\begin{aligned}
\mathbf{e}_{\tau \alpha_{1}}: 0= & -\Phi_{\tau \alpha_{1}}+\Phi_{n \alpha_{2}} \cos \left(\alpha_{1}\right)+\Phi_{\operatorname{cor}}\left(V_{\alpha_{2}}\right) \cos \left(\alpha_{1}\right) \\
& +\Phi_{n \varphi} \sin \left(\alpha_{2}+\theta\right) \cos \left(\alpha_{1}\right) \\
& +\Phi_{\tau \varphi} \cos \left(\alpha_{2}+\theta\right) \cos \left(\alpha_{1}\right)-\mathbf{m g} \sin \left(\alpha_{1}\right)=0 ; \\
\mathbf{e}_{\tau \alpha_{2}}: 0= & -\Phi_{\tau \alpha_{2}}-\Phi_{\operatorname{cor}}\left(V_{\alpha_{1}}\right) \\
& +\Phi_{n \varphi} \cos \left(\alpha_{2}+\theta\right)-\Phi_{\tau \varphi} \sin \left(\alpha_{2}+\theta\right),
\end{aligned}
$$

where

$$
\begin{aligned}
& \sin (\theta)=\frac{l \sin \left(\alpha_{1}\right) \cos \left(\alpha_{2}\right)}{\left\|O_{2} M\right\|} ; \\
& \cos (\theta)=\frac{\left(R+l \sin \left(\alpha_{1}\right) \sin \left(\alpha_{2}\right)\right)}{\left\|O_{2} M\right\|} .
\end{aligned}
$$

\section{N. Uniform Crane Boom Rotation Assumption with Nonlinear Equations in Relative Spherical Coordinates in the Noninertial Reference Frames $\mathscr{B}$}

Assuming formulae (G.1), we will have the following nonlinear ODE system in the noninertial reference frame $\mathscr{B}$, 
Relative trajectory of load $M$ swaying for nonlinear model

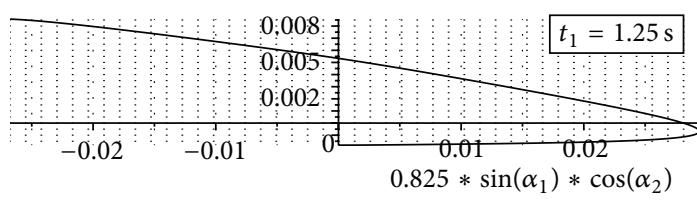

$y(x): l=0.825 \mathrm{~m} ; k \approx 3.448 \mathrm{rad} / \mathrm{s} ; \omega_{e} \approx 0.209 \mathrm{rad} / \mathrm{s}$

(a)

Relative trajectory of load $M$ swaying for nonlinear model

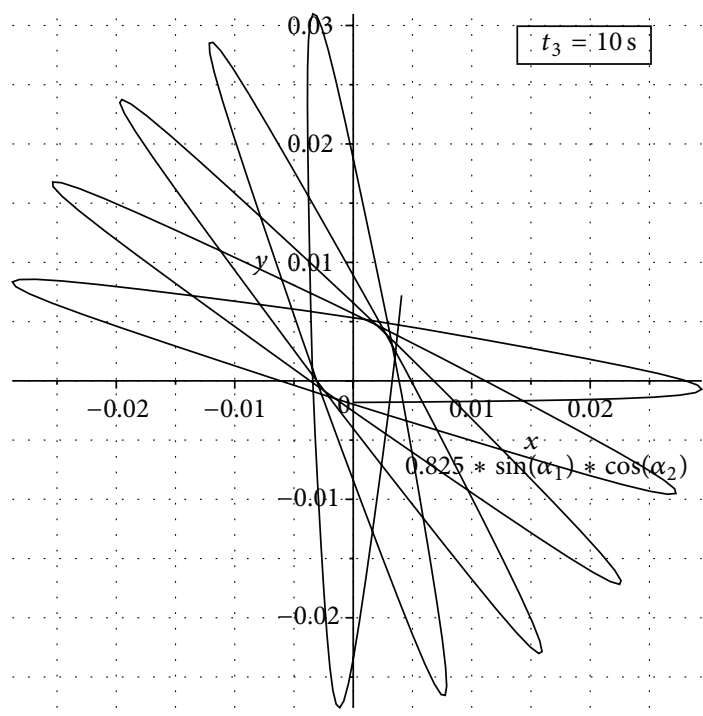

$-y(x): l=0.825 \mathrm{~m} ; k \approx 3.448 \mathrm{rad} / \mathrm{s} ; \omega_{e} \approx 0.209 \mathrm{rad} / \mathrm{s}$

(c)

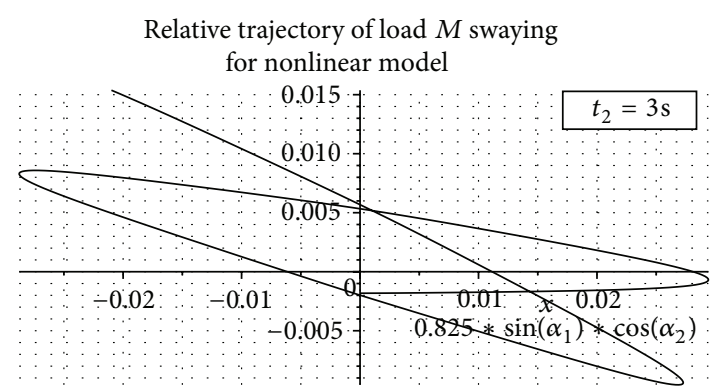

$y(x): l=0.825 \mathrm{~m} ; k \approx 3.448 \mathrm{rad} / \mathrm{s} ; \omega_{e} \approx 0.209 \mathrm{rad} / \mathrm{s}$

(b)

Relative trajectory of load $M$ swaying for nonlinear model

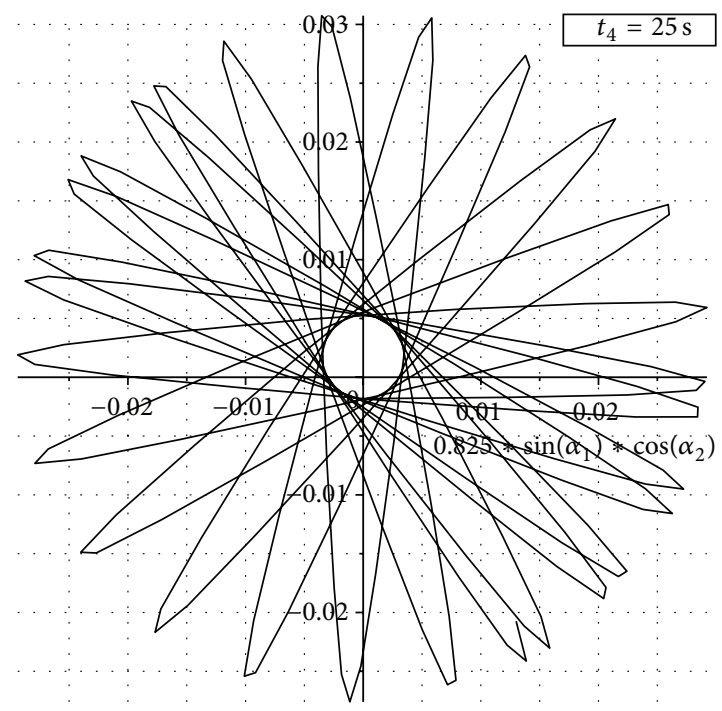

$y(x): l=0.825 \mathrm{~m} ; k \approx 3.448 \mathrm{rad} / \mathrm{s} ; \omega_{e} \approx 0.209 \mathrm{rad} / \mathrm{s}$

(d)

FiguRE 16: The relative trajectories for load $M$ swaying on the cable during uniform rotational motion of the crane boom $\mathrm{BO}_{2}$ for the moments of times $t_{1}=1.25 \mathrm{~s}(\mathrm{a}), t_{2}=3 \mathrm{~s}(\mathrm{~b}), t_{3}=10 \mathrm{~s}(\mathrm{c})$, and $t_{4}=25 \mathrm{~s}(\mathrm{~d})$, derived with nonlinear model introduction.

describing payload $M$ relative swaying in the case of uniform crane boom slewing:

$$
\begin{gathered}
\left(\frac{d^{2} \alpha_{1}}{d t^{2}}\right)-\frac{1}{2}\left(\frac{d \alpha_{2}}{d t}\right)^{2} \sin \left(2 \alpha_{1}\right)-\frac{1}{2} \omega_{e}^{2} \sin \left(2 \alpha_{1}\right) \\
-\left(\frac{R}{l}\right) \omega_{e}^{2} \cos \left(\alpha_{1}\right) \sin \left(\alpha_{2}\right)=-\left(\frac{g}{l}\right) \sin \left(\alpha_{1}\right) ; \\
\left(\frac{d^{2} \alpha_{2}}{d t^{2}}\right) \sin \left(\alpha_{1}\right)+2\left(\frac{d \alpha_{1}}{d t}\right)\left(\omega_{e}+\left(\frac{d \alpha_{2}}{d t}\right)\right) \cos \left(\alpha_{1}\right) \\
-\left(\frac{R}{l}\right) \omega_{e}^{2} \cos \left(\alpha_{2}\right)=0 .
\end{gathered}
$$

The initial conditions for the nonlinear system (N.1) may be formulated on the basis of the initial conditions in the noninertial reference frame $\mathscr{F}$ and with introduction of constraint equations (C.3):

$$
\begin{gathered}
l \sin \left(\alpha_{1}(0)\right) \cos \left(\alpha_{2}(0)\right)=0 ; \\
l \sin \left(\alpha_{1}(0)\right) \sin \left(\alpha_{2}(0)\right)=-y_{\mathrm{dyn}} ; \\
l\left(\frac{d \alpha_{1}(0)}{d t}\right) \cos \left(\alpha_{1}(0)\right) \cos \left(\alpha_{2}(0)\right) \\
-l\left(\frac{d \alpha_{2}(0)}{d t}\right) \sin \left(\alpha_{1}(0)\right) \sin \left(\alpha_{2}(0)\right)=\omega_{e} R ; \\
l\left(\frac{d \alpha_{1}(0)}{d t}\right) \cos \left(\alpha_{1}(0)\right) \sin \left(\alpha_{2}(0)\right) \\
+l\left(\frac{d \alpha_{2}(0)}{d t}\right) \sin \left(\alpha_{1}(0)\right) \cos \left(\alpha_{2}(0)\right)=0 .
\end{gathered}
$$




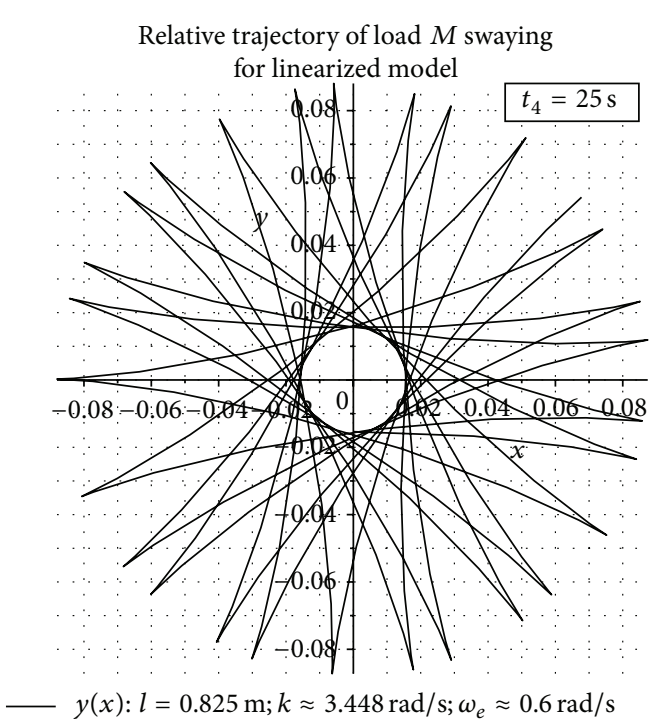

(a)

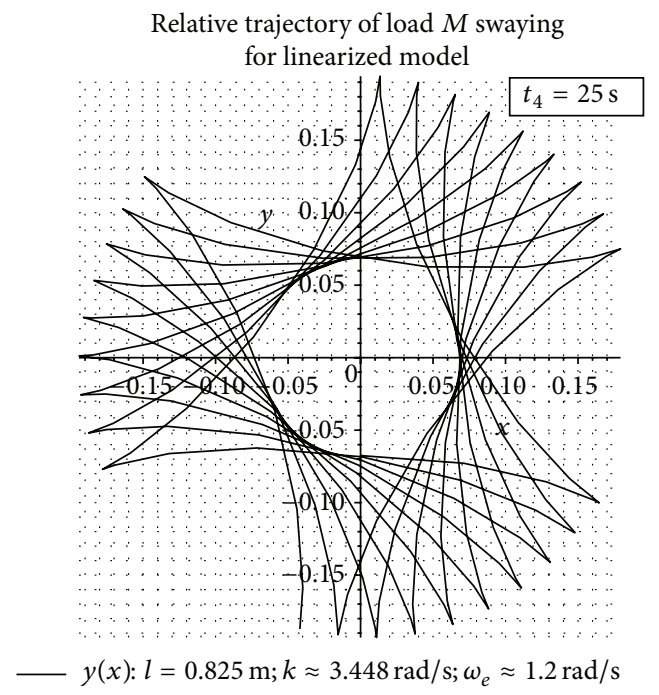

(c)

Relative trajectory of load $M$ swaying for linearized model

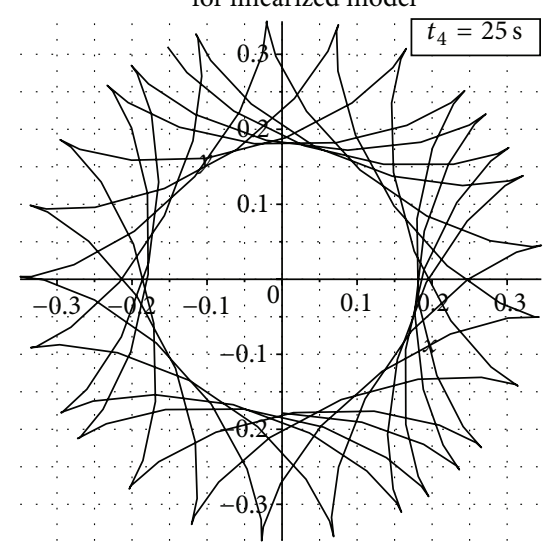

$y(x): l=0.825 \mathrm{~m} ; k \approx 3.448 \mathrm{rad} / \mathrm{s} ; \omega_{e} \approx 1.8 \mathrm{rad} / \mathrm{s}$

(e)

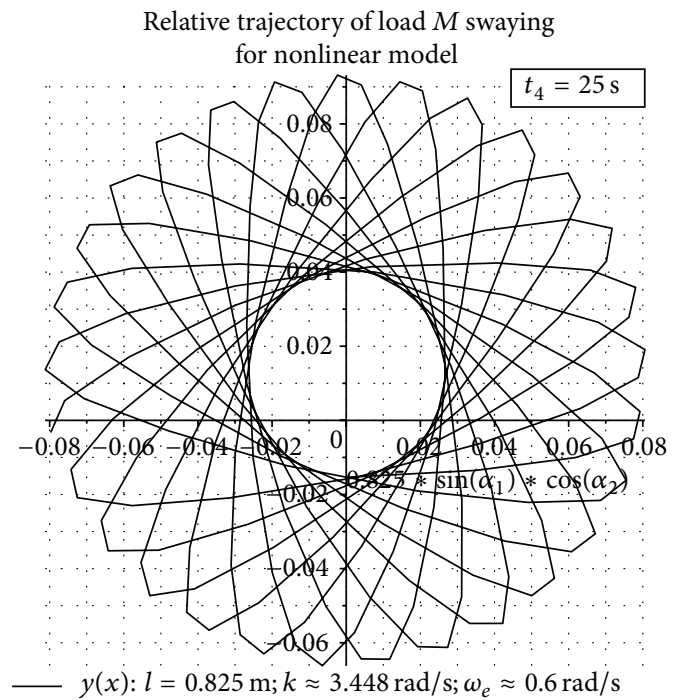

(b)

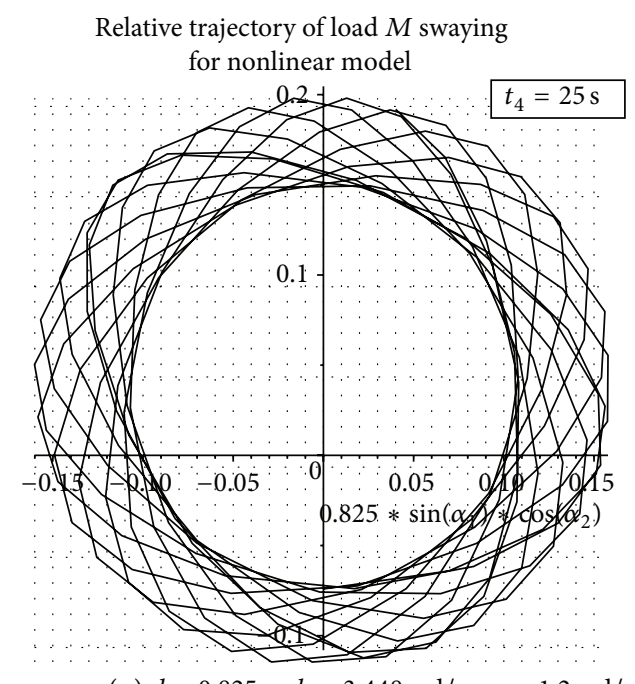

$y(x): l=0.825 \mathrm{~m} ; k \approx 3.448 \mathrm{rad} / \mathrm{s} ; \omega_{e} \approx 1.2 \mathrm{rad} / \mathrm{s}$

(d)

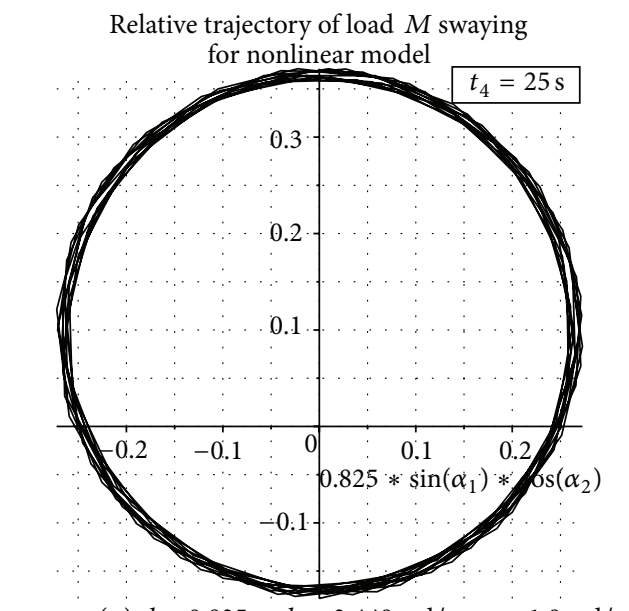

$y(x): l=0.825 \mathrm{~m} ; k \approx 3.448 \mathrm{rad} / \mathrm{s} ; \omega_{e} \approx 1.8 \mathrm{rad} / \mathrm{s}$

(f)

Figure 17: The comparison of relative trajectories of load $M$ swaying for linearized (a), (c), (e), and nonlinear (b), (d), and (f) models in the cases of $\omega_{e} / \omega_{e 0} \approx 2.871(\mathrm{a}-\mathrm{b}), \omega_{e} / \omega_{e 0} \approx 5.742(\mathrm{c}-\mathrm{d})$, and $\omega_{e} / \omega_{e 0} \approx 8.612(\mathrm{e}-\mathrm{f})$. 
Relative trajectory of load $M$ swaying for linearized model

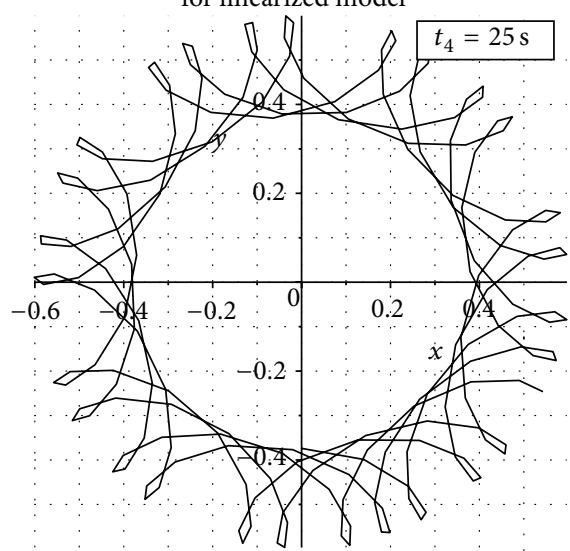

$y(x): l=0.825 \mathrm{~m} ; k \approx 3.448 \mathrm{rad} / \mathrm{s} ; \omega_{e} \approx 2.4 \mathrm{rad} / \mathrm{s}$

(a)

Relative trajectory of load $M$ swaying

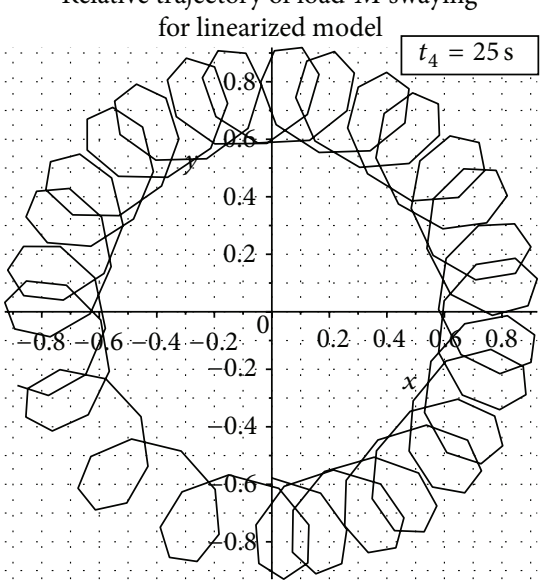

$y(x): l=0.825 \mathrm{~m} ; k \approx 3.448 \mathrm{rad} / \mathrm{s} ; \omega_{e} \approx 3.0 \mathrm{rad} / \mathrm{s}$

(c)

Relative trajectory of load $M$ swaying for linearized model

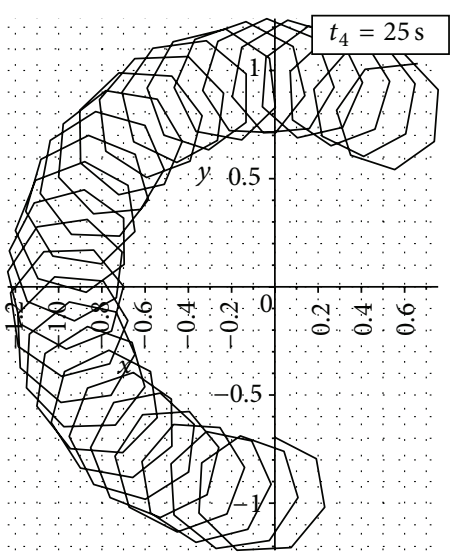

$y(x): l=0.825 \mathrm{~m} ; k \approx 3.448 \mathrm{rad} / \mathrm{s} ; \omega_{e} \approx 3.6 \mathrm{rad} / \mathrm{s}$

(e)
Relative trajectory of load $M$ swaying for nonlinear model

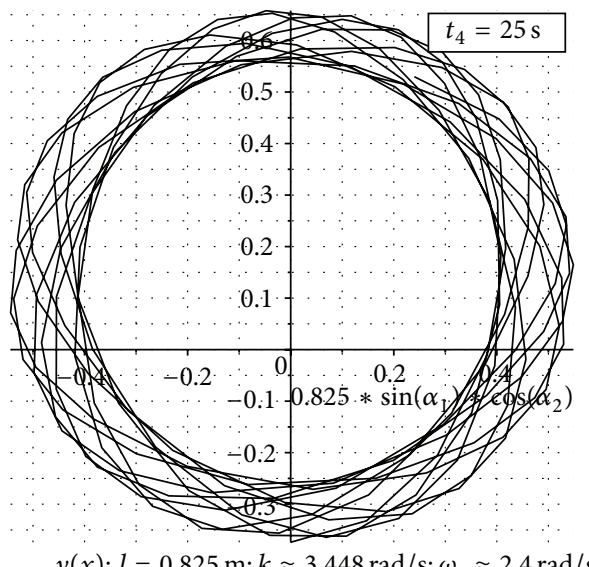

(b)

Relative trajectory of load $M$ swaying

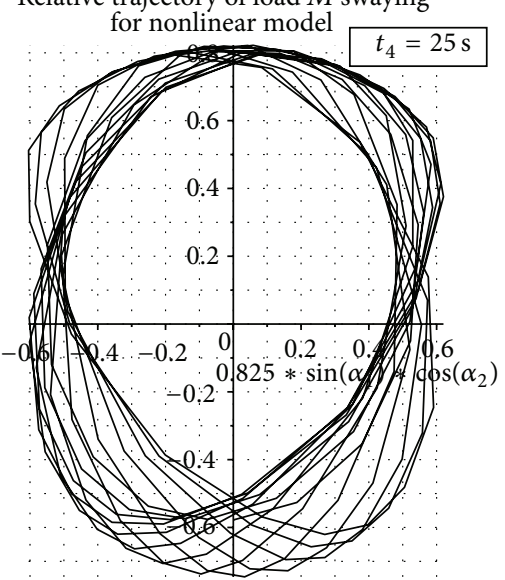

$y(x): l=0.825 \mathrm{~m} ; k \approx 3.448 \mathrm{rad} / \mathrm{s} ; \omega_{e} \approx 3.0 \mathrm{rad} / \mathrm{s}$

(d)

Relative trajectory of load $M$ swaying for nonlinear model

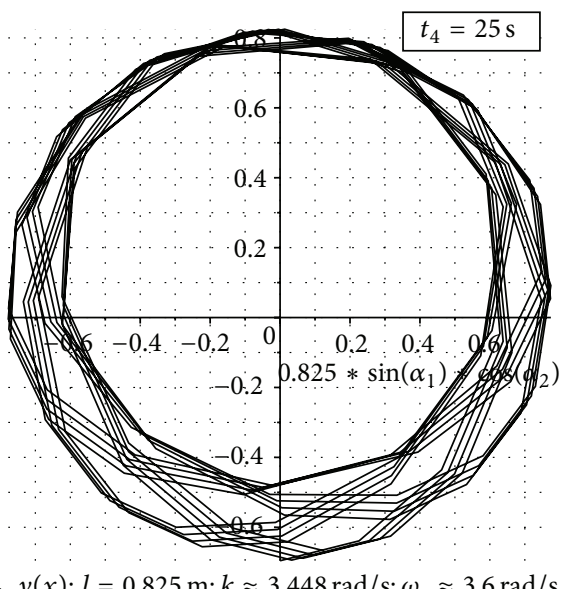

(f)

Figure 18: The comparison of relative trajectories of load $M$ swaying for linearized (a), (c), (e), and nonlinear (b), (d), and (f) models in the cases of $\omega_{e} / \omega_{e 0} \approx 11.483(\mathrm{a}-\mathrm{b}), \omega_{e} / \omega_{e 0} \approx 14.354(\mathrm{c}-\mathrm{d})$, and $\omega_{e} / \omega_{e 0} \approx 17.225$ (e-f). 
The solution of the system (N.2) defines the initial conditions for the nonlinear ODE system (N.1) for payload spherical coordinates' $\alpha_{1}$ and $\alpha_{2}$. The solution set member of the system (N.2) has the following form:

$$
\begin{aligned}
& \alpha_{1}(0)=\arcsin \left(\frac{y_{\mathrm{dyn}}}{l}\right) ; \\
& \alpha_{2}(0)=-\frac{\pi}{2} \\
& \frac{d \alpha_{1}(0)}{d t}=0 ; \\
& \frac{d \alpha_{2}(0)}{d t}=\frac{\omega_{e} R}{y_{\mathrm{dyn}}} .
\end{aligned}
$$

The numerical solution of the Cauchy problem (N.1)(N.3) for the nonlinear problem in spherical coordinates determines the shape of the relative payload $M$ swaying trajectory as is shown in Figures 16, 17(b), 17(d), 17(f), 18(b), 18(d), and 18(f). Numerical plots in Figure 16 have been carried out for the following values of mechanical system parameters: $R=0.492 \mathrm{~m} ; g=9.81 \mathrm{~m} / \mathrm{s}^{2} ; l=0.825 \mathrm{~m}$; $k=(g / l)^{0.5} \approx 3.448 \mathrm{rad} / \mathrm{s} ; T=30 \mathrm{~s} ; \omega_{e}=2 \pi / T \approx 0.209 \mathrm{rad} / \mathrm{s} ;$ $\varphi_{e}=180^{\circ} ; \alpha_{1}(0) \approx 0.00221 \mathrm{rad} ; \alpha_{2}(0) \approx-1.57079 \mathrm{rad} ;$ $V_{B}=0.103 \mathrm{~m} / \mathrm{s} ; y_{\mathrm{dyn}}=0.00182 \mathrm{~m} ; d\left(\alpha_{1}(0)\right) / d t=0 \mathrm{rad} / \mathrm{s}$; and $d\left(\alpha_{2}(0)\right) / d t=56.565608 \mathrm{rad} / \mathrm{s}$ (Figure 16).

\section{O. Comparative Estimation and Discussion of the Amplitude Responses, Computed from the Linear and Nonlinear Models}

For comparison of the linear (H.4)-(H.5) and nonlinear (N.1) models, we introduce the relative dimensionless amplitude discrepancy $\delta_{1}$ between averaged radii $r_{\text {nonlinear }}$ (Figures 17(b), 17(d), 17(f), 18(b), 18(d), and 18(f)) and $r_{\text {linearized }}$ (Figures 17(a), 17(c), 17(e), 18(a), 18(c), and 18(e)) of computational relative trajectories of payload $M$ in the noninertial reference frame $\mathscr{F}$ (Figures 17-18):

$$
\begin{aligned}
\delta_{1}=\left\langle\frac{1}{2}(\right. & \left(\frac{\left|r_{\text {nonlinear }}-r_{\text {linearized }}\right|}{r_{\text {nonlinear }}}\right) \\
& \left.\left.+\left(\frac{\left|r_{\text {nonlinear }}-r_{\text {linearized }}\right|}{r_{\text {linearized }}}\right)\right)\right\rangle .
\end{aligned}
$$

The results of numerical amplitude comparison, computed from the linear and nonlinear models in accordance with the formula (O.1) and Figures 12,16, 17, and 18, are shown in Figure 19.

The computational relative trajectories of load $M$ in the noninertial reference frame $\mathscr{F}$, shown in Figures 12, 16, 17 and 18 outline that both averaged radii $r_{\text {nonlinear }}$ (Figures $17(\mathrm{~b})$, 17(d), 17(f), 18(b), 18(d) and 18(f)) and $r_{\text {linearized }}$ (Figures 17(a), 17(c), 17(e), 18(a), 18(c), and 18(e)) and amplitudes of relative trajectories increase with increasing dimensionless crane boom slewing velocity $\omega_{e} / \omega_{e 0}$, where $\omega_{e 0} \approx 0.209 \mathrm{rad} / \mathrm{s}$ is

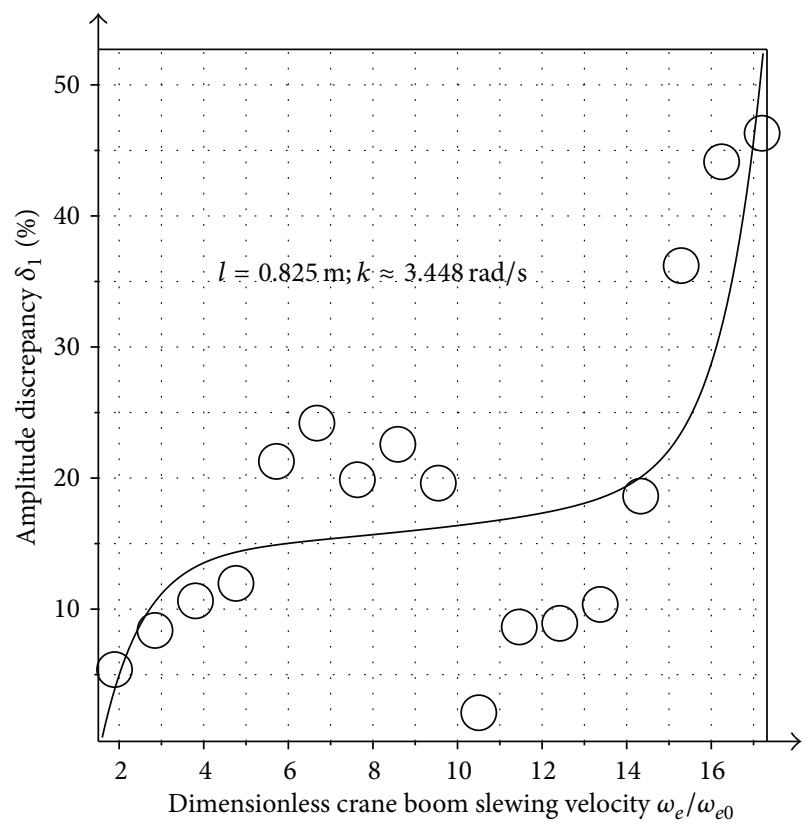

FIgURE 19: The computational points (O०૦०) and regression diagram (-) for the relative dimensionless amplitude discrepancy $\delta_{1}$ between averaged radii of computational relative trajectories of payload $M$ in the noninertial reference frame $\mathscr{F}$, derived for linearized and nonlinear models in the case of cable length $l=$ $0.825 \mathrm{~m}$

the experimentally chosen technological crane boom slewing velocity.

It is shown in Figures 12, 16, 17, and 18 that there is a persistent trend $r_{\text {linearized }}>r_{\text {nonlinear }}$.

The computational diagram in Figure 19 shows that the relative dimensionless amplitude discrepancy $\delta_{1}$ between the linear and nonlinear models increases with increasing dimensionless crane boom slewing velocity $\omega_{e} / \omega_{e 0}$. It is shown in Figure 19 that $\delta_{1}<11 \%$ for the $\omega_{e}<0.8 \mathrm{rad} / \mathrm{s}$, that is, for $\omega_{e}<3.828 \omega_{e 0}$. It is shown in Figure 19 that $\delta_{1}<11 \%$ for the $\alpha_{1}<0.145 \mathrm{rad}$.

So we draw a conclusion that the small angle assumption for the linearized model (H.4)-(H.5) adequately describes nonlinear load swaying problem (N.1) during uniform crane boom slewing for the above mentioned limiting values of $\omega_{e}$ and $\alpha_{1}$.

\section{Nomenclature}

DOF:

ODE:

DAE:

point $A_{\mathrm{st}}$ :

point $A_{\text {dyn }}$ :

point $M$ :
Degree of freedom

Ordinary differential equation Differential algebraic equation Point of static equilibrium of payload $M$ at the cable $B M$ Point of dynamic equilibrium of payload $M$ at the cable $B M$ Point of position of payload $M$ in the current moment of time 
point $E$ :

point $B$ :

point $D$ :

$m:$

$g:$

$\pi$

$C_{1} ; C_{2} ; C_{3}$ and $C_{4}: \quad$ Constants of integration (m)

$\lambda_{1} ; \lambda_{2}$ : Roots of secular equation

$t:$

$\mathscr{E}:$

$\widehat{\mathbf{e}}_{1} ; \widehat{\mathbf{e}}_{2} ; \widehat{\mathbf{e}}_{3}:$

$x_{2} ; y_{2} ; z_{2}$

$\mathscr{B}:$

$x_{1} ; y_{1} ; z_{1}$ :

$\widetilde{\mathbf{e}}_{1} ; \widetilde{\mathbf{e}}_{2} ; \widetilde{\mathbf{e}}_{3}:$

$\mathscr{F}:$

$\mathbf{e}_{1} ; \mathbf{e}_{2} ; \mathbf{e}_{3}$ :

$\mathscr{C}:$

$\mathbf{e}_{\tau \alpha_{1}} ; \mathbf{e}_{n \alpha_{1}} ; \mathbf{e}_{b \alpha_{1}}:$

D:

$\mathbf{e}_{\tau \alpha_{2}} ; \mathbf{e}_{n \alpha_{2}} ; \mathbf{e}_{b \alpha_{2}}:$

$\mathscr{G}$

$$
\mathbf{e}_{\tau \varphi} ; \mathbf{e}_{n \varphi} ; \mathbf{e}_{b \varphi}:
$$

$\mathbf{r}_{B / M}:$
Initial point of crane boom $B E$

Terminal point of crane boom $B E$

Center of circle for trajectory of transport and absolute motion for the point $B$

Mass of payload $M(\mathrm{~kg})$

Scalar value of gravitational

acceleration $\left(\mathrm{m} / \mathrm{s}^{2}\right)$

Ratio of perimeter of circle to

diameter

Roots of secular equation
Swaying time for payload $M(\mathrm{~s})$

Inertial reference frame $\left(\mathrm{O}_{2} x_{2} y_{2} z_{2}\right)$

fixed on earth

Orthogonal unit vectors defining the

inertial reference frame $\mathscr{E}$

The absolute coordinates of payload

$M$ with respect to Earth (m)

Noninertial reference frame

$\left(O_{1} x_{1} y_{1} z_{1}\right)$ which is connected with

rotating crane boom $\mathrm{O}_{2} B$ and the

origin of coordinates point $O_{1}$

coincides with point $A_{\text {st }}$ of static

equilibrium for load $M$ at the cable

\section{$B M$}

The relative coordinates of payload $M$

with respect to crane boom $\mathrm{O}_{2} \mathrm{~B}(\mathrm{~m})$

The orthogonal unit vectors defining

the noninertial reference frame $\mathscr{B}$

Noninertial reference frame $(O x y z)$

with coordinate axes parallel to

$\left(O_{1} x_{1} y_{1} z_{1}\right)$ and the origin of

coordinates point $O$ coincides with

point $A_{\mathrm{dyn}}$ of dynamic equilibrium

for load $M$ at the cable $B M$

Orthogonal unit vectors defining the

noninertial reference frame $\mathscr{F}$

Natural noninertial comoving

reference frame which corresponds

to the swaying angle $\alpha_{1}$ and moving

together with payload $M$

Orthogonal unit vectors defining the

noninertial reference frame $\mathscr{C}$;

Natural noninertial comoving

reference frame which corresponds

to the swaying angle $\alpha_{2}$ and moving

together with payload $M$

Orthogonal unit vectors defining the

noninertial reference frame $\mathscr{D}$

Natural noninertial comoving

reference frame which corresponds to

the angle of crane boom rotation $\varphi_{e}$

and moving together with payload $M$

Orthogonal unit vectors defining the

noninertial reference frame $\mathscr{G}$

Position vector, connecting initial

point $B$ and terminal point $M$ in

noninertial reference frame $\mathscr{B}(\mathrm{m})$

$$
\begin{aligned}
& \mathbf{r}_{\mathrm{O}_{2} / B} \text { : } \\
& R=B D: \\
& l=l_{B M}=\left\|\mathbf{r}_{B / M}\right\|: \quad \text { Length of the cable, that is, the } \\
& \text { magnitude of the position vector } \\
& \mathbf{r}_{B / M}(\mathrm{~m}) \\
& k=(g / l)^{0.5}: \\
& \tau=2 \pi / k: \\
& \varphi_{e}=L\left(\widehat{\mathbf{e}}_{1}, \widetilde{\mathbf{e}}_{2}\right): \\
& \gamma: \\
& \alpha_{1}=\angle\left(\widetilde{\mathbf{e}}_{3}, \mathbf{r}_{B / M}\right) \\
& \alpha_{2} \\
& \beta=\angle\left(A_{t} B A_{\mathrm{dyn}}\right): \\
& \theta: \\
& \text { Natural frequency of undamped } \\
& \text { oscillations of payload } M \text { at the cable } \\
& B M(1 / s) \\
& \text { Period of undamped oscillations of } \\
& \text { payload } M \text { at the cable } B M \text { (s) } \\
& \text { Angle of rotation of noninertial } \\
& \text { reference frame } \mathscr{B} \text { around axis } \mathrm{O}_{2} z_{2} \\
& \text { of inertial reference frame } \mathscr{E} \text {, that is, } \\
& \text { the angle of transport rotation for } \\
& \text { crane boom } \mathrm{BO}_{2} \text { around the vertical } \\
& \text { axis } \mathrm{O}_{2} z_{2} \text {, that is, crane boom slewing } \\
& \text { angle (rad) } \\
& \text { Angle between the position vector } \\
& \mathbf{r}_{\mathrm{O}_{2} / B} \text { and horizontal plane }\left(\widehat{\mathbf{e}}_{1}, \widehat{\mathbf{e}}_{2}\right) \text { or } \\
& \left(\widetilde{\mathbf{e}}_{1}, \widetilde{\mathbf{e}}_{2}\right) \text {, that is, inclination angle of } \\
& \text { crane boom } \mathrm{O}_{2} B \text { with horizontal } \\
& \text { plane }\left(\widehat{\mathbf{e}}_{1}, \widehat{\mathbf{e}}_{2}\right) \text { or }\left(\widetilde{\mathbf{e}}_{1}, \widetilde{\mathbf{e}}_{2}\right)(\mathrm{rad}) \\
& \text { Swaying angle between the vertical } \\
& \text { axis } O_{1} z_{1} \text { and the position vector } \\
& \mathbf{r}_{B / M} \text {, that is, the first spherical } \\
& \text { coordinate of spherical pendulum } M \\
& \text { (rad) } \\
& \text { Swaying angle between the planes } \\
& \left(x_{1} z_{1}\right) \text { and }\left(B M O_{1}\right) \text {, that is, the } \\
& \text { second spherical coordinate of } \\
& \text { spherical pendulum } M \text { (rad) } \\
& \text { Angle of ascent of payload } M \text { over } \\
& \text { dynamical equilibrium level } A_{\text {dyn }} \\
& \text { (rad) } \\
& \text { Angle between the vertical plane, } \\
& \text { connected with crane boom } \mathrm{O}_{2} \mathrm{~B} \text {, and } \\
& \text { the vertical plane, connected with } \\
& \text { position vector } \mathbf{r}_{\mathrm{O}_{2} / M} \text { of payload } M \\
& \text { in inertial reference frame } \mathscr{E}(\mathrm{rad}) \\
& h=l\left(1-\cos \left(\alpha_{1}\right)\right)=\text { Vertical coordinate of payload in } \\
& z_{1}: \quad \text { noninertial reference frame } \mathscr{B}(\mathrm{m}) \\
& \alpha_{1 \mathrm{dyn}} \text { : } \quad \text { Angular distance between the axis } \\
& A_{\mathrm{st}} z_{1} \text { and the line } B A_{\mathrm{dyn}}(\mathrm{rad}) \\
& y_{\text {dyn }}=l \sin \left(\alpha_{1 \mathrm{dyn}}\right): \quad \text { Length of perpendicular from point } \\
& A_{\text {dyn }} \text { to the axis } A_{\text {st }} z_{1}(\mathrm{~m}) \\
& \Delta=\quad \text { Vertical distance between the point } \\
& l\left(1-\cos \left(\alpha_{1 \mathrm{dyn}}\right)\right): \quad A_{\mathrm{st}} \text { and the point } A_{\mathrm{dyn}} \text {, that is, the } \\
& \text { vertical distance between the } \\
& \text { horizontal planes }\left(\widetilde{\mathbf{e}}_{1}, \widetilde{\mathbf{e}}_{2}\right) \text { and }\left(\mathbf{e}_{1}, \mathbf{e}_{2}\right) \\
& \text { of the noninertial reference frames } \mathscr{B} \\
& { }^{\mathscr{E}} \boldsymbol{\omega}^{\mathscr{B}}=\boldsymbol{\omega}_{\mathscr{B} / \mathscr{E}} \\
& \text { and } \mathscr{F} \text { correspondingly }(\mathrm{m})
\end{aligned}
$$




$$
\begin{aligned}
& \mathscr{E}^{\mathscr{F}}=\boldsymbol{\omega}_{\mathscr{F} / \mathscr{E}}: \\
& \omega_{\mathscr{B} / \mathscr{E}}=\omega_{\mathscr{F} / \mathscr{E}}= \\
& \omega_{e}=d \varphi_{e} / d t: \\
& \boldsymbol{\omega}_{\mathscr{B} / \mathscr{E}}=\boldsymbol{\omega}_{\mathscr{F} / \mathscr{C}}= \\
& \boldsymbol{\omega}_{e}=\left(d \varphi_{e} / d t\right) \widehat{\mathbf{e}}_{3}: \\
& \mathscr{E}_{\mathcal{E}} \mathscr{B}=\boldsymbol{\varepsilon}_{\mathscr{B} / \mathscr{E}}= \\
& \boldsymbol{\alpha}_{\mathscr{B} / \mathscr{E}}: \\
& \mathscr{E}_{\mathcal{E}}{ }^{\mathscr{F}}=\boldsymbol{\varepsilon}_{\mathscr{F} / \mathscr{E}}= \\
& \boldsymbol{\alpha}_{\mathscr{F} / \mathscr{E}}: \\
& \alpha_{\mathscr{B} / \mathscr{E}}=\alpha_{\mathscr{F} / \mathscr{E}}= \\
& \mathcal{\varepsilon}_{e}=d^{2} \varphi_{e} / d t^{2}:
\end{aligned}
$$

Angular velocity vector of reference frame $\mathscr{F}$ with respect to frame $\mathscr{E}$ $(\mathrm{rad} / \mathrm{s})$

Scalar of transport angular velocity of reference frames $\mathscr{B}$ and $\mathscr{F}$ with respect to frame $\mathscr{E}(\mathrm{rad} / \mathrm{s})$

Transport angular velocity vector of reference frames $\mathscr{B}$ and $\mathscr{F}$ with respect to frame $\mathscr{E}(\mathrm{rad} / \mathrm{s})$

Angular acceleration vector of reference frame $\mathscr{B}$ with respect to frame $\mathscr{E}\left(\mathrm{rad} / \mathrm{s}^{2}\right)$

Angular acceleration vector of reference frame $\mathscr{F}$ with respect to frame $\mathscr{E}\left(\mathrm{rad} / \mathrm{s}^{2}\right)$

Scalar of transport angular acceleration of reference frames $\mathscr{B}$ and $\mathscr{F}$ with respect to frame $\mathscr{E}$ $\left(\mathrm{rad} / \mathrm{s}^{2}\right)$

$\boldsymbol{\varepsilon}_{\mathscr{B} / \mathscr{C}}=\boldsymbol{\varepsilon}_{\mathscr{F} / \mathscr{C}}=\boldsymbol{\varepsilon}_{\mathrm{e}}=$ Transport angular acceleration vector $\left(d^{2} \varphi_{e} / d t^{2}\right) \mathbf{e}_{3}$ :

${ }^{\mathscr{E}} \mathbf{V}^{M}=\mathbf{V}_{M / \mathscr{E}}=$ $\mathbf{V}_{\mathrm{abs}}$ : of reference frames $\mathscr{B}$ and $\mathscr{F}$ with respect to frame $\mathscr{E}\left(\mathrm{rad} / \mathrm{s}^{2}\right)$

Velocity of point $M$ in inertial fixed on earth reference frame $\mathscr{E}$, that is, absolute velocity of payload $M(\mathrm{~m} / \mathrm{s})$

${ }^{\mathscr{B}} \mathbf{V}^{M}=\mathbf{V}_{M / \mathscr{B}}=$

${ }^{\mathscr{F}} \mathbf{V}^{M}=\mathbf{V}_{M / \mathscr{F}}=$

$\mathrm{V}_{\mathbf{r}}$ :

$\mathbf{V}_{\mathbf{e}}=\mathbf{V}_{\mathrm{O}_{2} / \mathscr{E}}+$

$\boldsymbol{\omega}_{\mathscr{B} / \mathscr{E}} \times \mathbf{r}_{\mathrm{O}_{2} / M}$ :

$V_{x_{1}} ; V_{y_{1}} ; V_{z_{1}}$ :

$V_{x} ; V_{y} ; V_{z}:$

${ }_{\mathscr{E}} \mathbf{a}^{M}=\mathbf{a}_{M / \mathscr{E}}=\mathbf{a}_{\text {abs }}:$ Acceleration of point $M$ in inertial Velocity of point $M$ in noninertial reference frames $\mathscr{B}$ and $\mathscr{F}$, that is, relative velocity of payload $M(\mathrm{~m} / \mathrm{s})$

Transport velocity of point $M$ in inertial reference frame $\mathscr{E}(\mathrm{m} / \mathrm{s})$

$x_{1^{-}}, y_{1^{-}}, z_{1}$-projections of payload $M$ velocity in noninertial reference frame $\mathscr{B}(\mathrm{m} / \mathrm{s})$

$x$-, $y$-, $z$-projections of payload $M$ velocity in noninertial reference frame $\mathscr{F}(\mathrm{m} / \mathrm{s})$ fixed on earth reference frame $\mathscr{E}$, that is, absolute acceleration of payload $M$ $\left(\mathrm{m} / \mathrm{s}^{2}\right)$

$$
\begin{aligned}
& { }_{\mathscr{B}}^{\mathbf{a}^{M}}=\mathbf{a}_{M / \mathscr{B}}= \\
& { }_{\mathscr{F}} \mathbf{a}^{M}=\mathbf{a}_{M / \mathscr{F}}=\mathbf{a}_{\mathbf{r}}: \\
& { }_{\mathscr{E}} \mathbf{a}^{E}=\mathbf{a}_{E / \mathscr{E}}= \\
& { }_{\mathscr{E}} \mathbf{a}^{D}=\mathbf{a}_{D / \mathscr{E}}=\mathbf{0}:
\end{aligned}
$$

Acceleration of point $M$ in noninertial reference frames $\mathscr{B}$ and $\mathscr{F}$, that is, relative acceleration of payload $M\left(\mathrm{~m} / \mathrm{s}^{2}\right)$

Acceleration of points $E$ and $D$ in inertial fixed on earth reference frame $\mathscr{E}$, that is, the absolute acceleration of points $E$ and $D$, located at rotation axis $E D\left(\mathrm{~m} / \mathrm{s}^{2}\right)$

$\mathbf{a}_{\mathbf{e}}{ }^{\boldsymbol{\tau}}=\boldsymbol{\alpha}_{\mathscr{B} / \mathscr{C}} \times \mathbf{r}_{E / M}:$ Tangential acceleration of transportation for payload $M\left(\mathrm{~m} / \mathrm{s}^{2}\right)$

$T:$ Kinetic energy of the system "crane boom $B \mathrm{O}_{2}$-load $M^{\prime \prime}(\mathrm{J}=\mathrm{N}-\mathrm{m})$

$\Pi$ :
Potential energy of the system "crane boom $B_{2}$-load $M^{\prime \prime}(\mathrm{J}=\mathrm{N}-\mathrm{m})$
$Q_{x_{1}} ; Q_{y_{1}} ; Q_{z_{1}}: \quad$ Generalized forces $(\mathrm{N})$

$v_{1} ; v_{2}$ :

$\tau_{1} ; \tau_{2}$

mg:

$\mathrm{N}$ :

$\boldsymbol{\Phi}_{\mathbf{e}}{ }^{\boldsymbol{\tau}}=(-m) \mathbf{a}_{\mathbf{e}}{ }^{\boldsymbol{\tau}}:$

First and second natural frequencies of payload relative oscillations $(1 / \mathrm{s})$

First and second periods of payload relative oscillations (s)

Gravitational force (N)

Reaction force of the cable $B M(\mathrm{~N})$

Tangential inertial force (tangential force of moving space) for payload $M$ (N)

$\boldsymbol{\Phi}_{\mathbf{e}}{ }^{\mathbf{n}}=(-m) \mathbf{a}_{\mathbf{e}}{ }^{\boldsymbol{\tau}}: \quad$ Normal or centrifugal inertial force (normal force of moving space) for payload $M(\mathrm{~N})$

$\Phi_{\text {cor }}=(-m) \mathbf{a}_{\text {cor }}$

$I_{33}^{\mathrm{O}_{2}}$ :

Coriolis inertial force (compound centrifugal force) for payload $M(\mathrm{~N})$

Element of mass moment of inertia for the system "crane boom $\mathrm{BO}_{2}$-load $M$ " in inertial fixed on earth reference frame $\mathscr{E}$ with respect to axis $\widehat{\mathbf{e}}_{3}\left(\mathrm{~kg}-\mathrm{m}^{2}\right)$

$\mathbf{H}_{3}^{\mathrm{O}_{2}}=I_{33}^{\mathrm{O}_{2}} \boldsymbol{\omega}_{\mathscr{B} / \mathscr{C}}:$

Moment of momentum for the system "crane boom $\mathrm{BO}_{2}$-load $\mathrm{M}$ ", that is, vector component of the angular momentum for "crane boom $\mathrm{BO}_{2}$-load $M$ " with respect to point $\mathrm{O}_{2}$ in inertial fixed on earth reference frame $\mathscr{E}\left(\mathrm{kg}-\mathrm{m}^{2} / \mathrm{s}\right)$

$\sum \mathbf{M}^{\mathrm{O}_{2}}=M^{\mathrm{O}_{2}} \widehat{\mathbf{e}}_{3}:$

Vector of the resultant external moment codirectional to axis $\widehat{\mathbf{e}}_{3}$ with respect to point $\mathrm{O}_{2}$, where point $\mathrm{O}_{2}$ is fixed in the inertial fixed on earth reference frame $\mathscr{E}(\mathrm{N}-\mathrm{m})$

$M_{D T}^{\mathrm{O}_{2}}$ : $\mathbf{M}_{D T}^{\mathrm{O}_{2}}=M_{D T}^{\mathrm{O}_{2}} \widehat{\mathbf{e}}_{3}:$

Driving torque for the system "crane boom $\mathrm{BO}_{2}$-load $M$ " with respect to point $\mathrm{O}_{2}$, where point $\mathrm{O}_{2}$ is fixed in the inertial fixed on earth reference frame $\mathscr{E}(\mathrm{N}-\mathrm{m})$

Vector of the driving torque for the system "crane boom $\mathrm{BO}_{2}$-load $M$ " codirectional to axis $\widehat{\mathbf{e}}_{3}$ with respect to point $\mathrm{O}_{2}$, where point $\mathrm{O}_{2}$ is fixed in the inertial fixed on earth reference frame $\mathscr{E}$ with respect to axis $\widehat{\mathbf{e}}_{3}(\mathrm{~N}-\mathrm{m})$

$M_{F T}^{\mathrm{O}_{2}}$ :

Frictional torque for the system "crane boom $\mathrm{BO}_{2}$-load $M$ " with respect to point $\mathrm{O}_{2}$, where point $\mathrm{O}_{2}$ is fixed in the inertial fixed on earth reference frame $\mathscr{E}(\mathrm{N}-\mathrm{m})$

$\mathbf{M}_{F T}^{\mathrm{O}_{2}}=M_{F T}^{\mathrm{O}_{2}} \widehat{\mathbf{e}}_{3}:$

Vector of the frictional torque for the system "crane boom $\mathrm{BO}_{2}$-load $M$ " codirectional to axis $\widehat{\mathbf{e}}_{3}$ with respect to point $\mathrm{O}_{2}$, where point $\mathrm{O}_{2}$ is fixed in the inertial fixed on earth reference frame $\mathscr{E}$ with respect to axis $\widehat{\mathbf{e}}_{3}(\mathrm{~N}-\mathrm{m})$ 


\section{$\delta: \quad$ Relative dimensionless amplitude discrepancy between computational and experimental absolute trajectories of payload $M$ in the inertial reference frame $\mathscr{E}$ \\ $\delta_{1}:$

\begin{abstract}
Relative dimensionless amplitude discrepancy between averaged radii of computational relative trajectories of payload $M$ in the noninertial reference frame $\mathscr{F}$, derived for linearized and nonlinear models.
\end{abstract}

\section{Disclosure}

The submission of the authors' paper implies that it has not been previously published, that it is not under consideration for publication elsewhere, and that it will not be published elsewhere in the same form without the written permission of the editors.

\section{Conflict of Interests}

The authors Alexander V. Perig, Alexander N. Stadnik, Alexander I. Deriglazov, and Sergey V. Podlesny declare that there is no conflict of interests regarding the publication of this paper.

\section{References}

[1] E. M. Abdel-Rahman and A. H. Nayfeh, "Pendulation reduction in boom cranes using cable length manipulation," Nonlinear Dynamics, vol. 27, no. 3, pp. 255-269, 2002.

[2] E. M. Abdel-Rahman, A. H. Nayfeh, and Z. N. Masoud, "Dynamics and control of cranes: a review," Journal of Vibration and Control, vol. 9, no. 7, pp. 863-908, 2003.

[3] E. M. Abdel-Rahman and A. H. Nayfeh, "Two-dimensional control for ship-mounted cranes: a feasibility study," Journal of Vibration and Control, vol. 9, no. 12, pp. 1327-1342, 2003.

[4] I. Adamiec-Wójcik, P. Fałat, A. Maczyński, and S. Wojciech, "Load Stabilisation in an a-frame-a type of an offshore crane," The Archive of Mechanical Engineering, vol. 56, no. 1, pp. 37-59, 2009.

[5] A. A. Al-mousa, A. H. Nayfeh, and P. Kachroo, "Control of rotary cranes using fuzzy logic," Shock and Vibration, vol. 10, no. 2, pp. 81-95, 2003.

[6] A. P. Allan and M. A. Townsend, "Motions of a constrained spherical pendulum in an arbitrarily moving reference frame: the automobile seatbelt inertial sensor," Shock and Vibration, vol. 2, no. 3, pp. 227-236, 1995.

[7] P. J. Aston, "Bifurcations of the horizontally forced spherical pendulum," Computer Methods in Applied Mechanics and Engineering, vol. 170, no. 3-4, pp. 343-353, 1999.

[8] P. Betsch, M. Quasem, and S. Uhlar, "Numerical integration of discrete mechanical systems with mixed holonomic and control constraints," Journal of Mechanical Science and Technology, vol. 23, no. 4, pp. 1012-1018, 2009.

[9] D. Blackburn, J. Lawrence, J. Danielson, W. Singhose, T. Kamoi, and A. Taura, "Radial-motion assisted command shapers for nonlinear tower crane rotational slewing," Control Engineering Practice, vol. 18, no. 5, pp. 523-531, 2010.

[10] D. Blackburn, W. Singhose, J. Kitchen et al., "Command shaping for nonlinear crane dynamics," JVC: Journal of Vibration and Control, vol. 16, no. 4, pp. 477-501, 2010.

[11] W. Blajer and K. Kołodziejczyk, "A geometric approach to solving problems of control constraints: theory and a DAE framework," Multibody System Dynamics, vol. 11, no. 4, pp. 343364, 2004.

[12] W. Blajer and K. Kołodziejczyk, "Dynamics and control of rotary cranes executing a load prescribed motion," Journal of Theoretical and Applied Mechanics, vol. 44, no. 4, pp. 929-948, 2006.

[13] W. Blajer and K. Kołodziejczyk, "Control of underactuated mechanical systems with servo-constraints," Nonlinear Dynamics, vol. 50, no. 4, pp. 781-791, 2007.

[14] W. Blajer and K. Kołodziejczyk, "Modeling of underactuated mechanical systems in partly specified motion," Journal of Theoretical and Applied Mechanics, vol. 46, no. 2, pp. 383-394, 2008.

[15] W. Blajer and K. Kołodziejczyk, "The use of dependent coordinates in modeling and simulation of cranes executing a load prescribed motion," The Archive of Mechanical Engineering, vol. 56, no. 3, pp. 209-222, 2009.

[16] W. Blajer, A. Czaplicki, K. Dziewiecki, and Z. Mazur, "Influence of selected modeling and computational issues on muscle force estimates," Multibody System Dynamics, vol. 24, no. 4, pp. 473492, 2010.

[17] W. Blajer and K. Kołodziejczyk, "Improved DAE formulation for inverse dynamics simulation of cranes," Multibody System Dynamics, vol. 25, no. 2, pp. 131-143, 2011.

[18] J.-H. Cha, S.-H. Ham, K.-Y. Lee, and M.-I. Roh, "Application of a topological modelling approach of multi-body system dynamics to simulation of multi-floating cranes in shipyards," Proceedings of the Institution of Mechanical Engineers K: Journal of Multi-Body Dynamics, vol. 224, no. 4, pp. 365-373, 2010.

[19] C. Chin, A. H. Nayfeh, and E. Abdel-Rahman, "Nonlinear dynamics of a boom crane," Journal of Vibration and Control, vol. 7, no. 2, pp. 199-220, 2001.

[20] K. Ellermann, E. Kreuzer, and M. Markiewicz, "Nonlinear dynamics of floating cranes," Nonlinear Dynamics, vol. 27, no. 2, pp. 107-183, 2002.

[21] T. Erneux and T. Kalmár-Nagy, "Nonlinear stability of a delayed feedback controlled container crane," Journal of Vibration and Control, vol. 13, no. 5, pp. 603-616, 2007.

[22] T. Erneux, "Strongly nonlinear oscillators subject to delay," Journal of Vibration and Control, vol. 16, no. 7-8, pp. 1141-1149, 2010.

[23] R. M. Ghigliazza and P. Holmes, "On the dynamics of cranes, or spherical pendula with moving supports," International Journal of Non-Linear Mechanics, vol. 37, no. 7, pp. 1211-1221, 2002.

[24] G. Glossiotis and I. Antoniadis, "Payload sway suppression in rotary cranes by digital filtering of the commanded inputs," Proceedings of the Institution of Mechanical Engineers K: Journal of Multi-body Dynamics, vol. 217, no. 2, pp. 99-109, 2003.

[25] B. Grigorov and R. Mitrev, "A Newton-Euler approach to the freely suspended load swinging problem," Recent Journal, vol. 10, no. 26, pp. 109-112, 2009.

[26] A. V. Gusev and M. P. Vinogradov, "Angular velocity of rotation of the swing plane of a spherical pendulum with an anisotropic suspension," Measurement Techniques, vol. 36, no. 10, pp. 10781082, 1993. 
[27] K.-S. Hong and Q. H. Ngo, "Dynamics of the container crane on a mobile harbor," Ocean Engineering, vol. 53, pp. 16-24, 2012.

[28] J . J . Hoon, K. Sung-Ha, and J. Eun Tae, "Pendulation reduction on ship-mounted container crane via T-S fuzzy model," Journal of Central South University, vol. 19, pp. 163-167, 2012.

[29] R. A. Ibrahim, "Excitation-induced stability and phase transition: a review," Journal of Vibration and Control, vol. 12, no. 10, pp. 1093-1170, 2006.

[30] B. Jerman, P. Podržaj, and J. Kramar, "An investigation of slewing-crane dynamics during slewing motion-development and verification of a mathematical model," International Journal of Mechanical Sciences, vol. 46, no. 5, pp. 729-750, 2004.

[31] B. Jerman, "An enhanced mathematical model for investigating the dynamic loading of a slewing crane," Proceedings of the Institution of Mechanical Engineers, Part C: Journal of Mechanical Engineering Science, vol. 220, no. 4, pp. 421-433.

[32] B. Jerman, "The comparison of the accuracy of two mathematical models, concerning dynamics of the slewing cranes," FME Transactions, vol. 34, pp. 185-192, 2006.

[33] B. Jerman and J. Kramar, "A study of the horizontal inertial forces acting on the suspended load of slewing cranes," International Journal of Mechanical Sciences, vol. 50, no. 3, pp. 490-500, 2008.

[34] F. Ju, Y. S. Choo, and F. S. Cui, "Dynamic response of tower crane induced by the pendulum motion of the payload," International Journal of Solids and Structures, vol. 43, no. 2, pp. 376-389, 2006.

[35] J. Kłosiński, "Fuzzy logic_-based control of a mobile crane slewing motion," Mechanics and Mechanical Engineering, vol. 15, no. 4, pp. 73-80, 2011.

[36] J. Krukowski, A. Maczyński, and M. Szczotka, "The influence of a shock absorber on dynamics of an offshore pedestal crane," Journal of Theoretical and Applied Mechanics, vol. 50, no. 4, pp. 953-966, 2012.

[37] J. Krukowski and A. Maczynski, "Application of the rigid finite element method for modeling an offshore pedestal crane," Archive of Mechanical Engineering, vol. 60, no. 3, pp. 451-471, 2013.

[38] S. Lenci, E. Pavlovskaia, G. Rega, and M. Wiercigroch, "Rotating solutions and stability of parametric pendulum by perturbation method," Journal of Sound and Vibration, vol. 310, no. 1-2, pp. 243-259, 2008.

[39] A. Y. T. Leung and J. L. Kuang, "On the chaotic dynamics of a spherical pendulum with a harmonically vibrating suspension," Nonlinear Dynamics, vol. 43, no. 3, pp. 213-238, 2006.

[40] V. S. Loveykin, Romasevich, and O. Yu, "Optimum management of dynamic systems taking into account higher derivates of management function," Scientific Herald National ForestTechnical University of Ukraine, vol. 21, no. 15, pp. 304-314, 2011 (Ukrainian).

[41] E. Maleki and W. Singhose, "Dynamics and control of a smallscale boom crane," Journal of Computational and Nonlinear Dynamics, vol. 6, no. 3, Article ID 031015, 8 pages, 2011.

[42] A. Maczynski and M. Szczotka, "Comparison of models for dynamic analysis of a mobile telescopic crane," Journal of Theoretical and Applied Mechanics, vol. 40, no. 4, pp. 1051-1074, 2002.

[43] A. Maczynski, "Load positioning and minimization of load oscillations in rotary cranes," Journal of Theoretical and Applied Mechanics, vol. 41, no. 4, pp. 873-885, 2003.

[44] A. Maczynski and S. Wojciech, "Dynamics of a mobile crane and optimisation of the slewing motion of its upper structure," Nonlinear Dynamics, vol. 32, no. 3, pp. 259-290, 2003.
[45] I. Marinović, D. Sprečić, and B. Jerman, "A slewing crane payload dynamics," Tehnički Vjesnik-Technical Gazette, vol. 19, no. 4, pp. 907-916, 2012.

[46] Z. N. Masoud, M. F. Daqaq, and N. A. Nayfeh, "Pendulation reduction on small ship-mounted telescopic cranes," Journal of Vibration and Control, vol. 10, no. 8, pp. 1167-1179, 2004.

[47] Z. N. Masoud, A. H. Nayfeh, and D. T. Mook, "Cargo pendulation reduction of ship-mounted cranes," Nonlinear Dynamics, vol. 35, no. 3, pp. 299-311, 2004.

[48] R. Mijailović, "Modelling the dynamic behaviour of the truckcrane," Transport, vol. 26, no. 4, pp. 410-417, 2011.

[49] R. Mitrev and B. Grigorov, "Dynamic behaviour of a spherical pendulum with spatially moving pivot," Zeszyty Naukowe Politechniki Poznańskiej, Budowa Maszyn i Zarządzanie Produkcja, vol. 9, pp. 81-91, 2008.

[50] R. Mitrev, "Mathematical modelling of translational motion of rail-guided cart with suspended payload," Journal of Zhejiang University SCIENCE A, vol. 8, no. 9, pp. 1395-1400, 2007.

[51] D. O. Morales, S. Westerberg, P. X. La Hera, U. Mettin, L. Freidovich, and A. S. Shiriaev, "Increasing the level of automation in the forestry logging process with crane trajectory planning and control," Journal of Field Robotics, vol. 31, no. 3, pp. 343-363, 2014.

[52] K. Nakazono, K. Ohnishi, and H. Kinjo, "Vibration control of load for rotary crane system using neural network with GAbased training," Artificial Life and Robotics, vol. 13, no. 1, pp. 98101, 2008.

[53] R. L. Neitzel, N. S. Seixas, and K. K. Ren, "A review of crane safety in the construction industry," Applied Occupational and Environmental Hygiene, vol. 16, no. 12, pp. 1106-1117, 2001.

[54] J. Neupert, E. Arnold, K. Schneider, and O. Sawodny, “Tracking and anti-sway control for boom cranes," Control Engineering Practice, vol. 18, no. 1, pp. 31-44, 2010.

[55] W. O'Connor and H. Habibi, "Gantry crane control of a doublependulum, distributed-mass load, using mechanical wave concepts," Mechanical Sciences, vol. 4, pp. 251-261, 2013.

[56] H. M. Omar and A. H. Nayfeh, "Anti-swing control of gantry and tower cranes using fuzzy and time-delayed feedback with friction compensation," Shock and Vibration, vol. 12, no. 2, pp. 73-89, 2005.

[57] M. Osiński and S. Wojciech, "Application of nonlinear optimisation methods to input shaping of the hoist drive of an off-shore crane," Nonlinear Dynamics, vol. 17, no. 4, pp. 369-386, 1998.

[58] F. Palis and S. Palis, "High performance tracking control of automated slewing cranes," in Robotics and Automation in Construction, C. Balaguer and M. Abderrahim, Eds., InTech, 2008.

[59] A. V. Perig, A. N. Stadnik, and A. I. Deriglazov, "Spherical pendulum small oscillations for slewing crane motion," The Scientific World Journal, vol. 2014, Article ID 451804, 10 pages, 2014.

[60] B. Posiadała, B. Skalmierski, and L. Tomski, "Motion of the lifted load brought by a kinematic forcing of the crane telescopic boom," Mechanism and Machine Theory, vol. 25, no. 5, pp. 547$556,1990$.

[61] H. L. Ren, X. L. Wang, Y. J. Hu, and C. G. Li, "Dynamic response analysis of a moored crane-ship with a flexible boom," Journal of Zhejiang University Science A, vol. 9, no. 1, pp. 26-31, 2008.

[62] D. Safarzadeh, S. Sulaiman, F. A. Aziz, D. B. Ahmad, and G. H. Majzoobi, "An approach to determine effect of crane hook on payload sway," International Journal of Mechanics and Applications, vol. 1, no. 1, pp. 30-38, 2011. 
[63] Y. Sakawa, Y. Shindo, and Y. Hashimoto, "Optimal control of a rotary crane," Journal of Optimization Theory and Applications, vol. 35 , no. 4 , pp. $535-557,1981$.

[64] O. Sawodny, J. Neupert, and E. Arnold, "Actual trends in crane automation-directions for the future," FME Transactions, vol. 37, pp. 167-174, 2009, http://www.mas.bg.ac.rs/_media/istrazivanje/fme/vol37/4/02_osawodny.pdf.

[65] M. P. Spathopoulos, D. Fragopoulos, A. Isidori, F. LamnabhiLagarrigue, and W. Respondek, "Control design of a crane for offshore lifting operations," in Nonlinear Control in the Year 2000, A. Isidori, F. Lamnabhi-Lagarrigue, and W. Respondek, Eds., vol. 2 of Lecture Notes in Control and Information Sciences, pp. 469-486, 2001.

[66] H. Schaub, "Rate-based ship-mounted crane payload pendulation control system," Control Engineering Practice, vol. 16, no. 1, pp. 132-145, 2008.

[67] W. Solarz and G. Tora, "Simulation of control drives in a tower crane," Transport Problems, vol. 6, no. 4, pp. 69-78, 2011, http://transportproblems.polsl.pl/pl/Archiwum/2011/zeszyt4/ 2011t6z4_08.pdf.

[68] N. Uchiyama, "Robust control for overhead cranes by partial state feedback," Proceedings of the Institution of Mechanical Engineers, Part I: Journal of Systems and Control Engineering, vol. 223 , no. 4 , pp. 575-580, 2009.

[69] A. Urbaś, "Analysis of flexibility of the support and its influence on dynamics of the grab crane," Latin American Journal of Solids and Structures, vol. 10, no. 1, pp. 109-121, 2013.

[70] E. Hairer, S. P. Nørsett, and G. Wanner, Solving Ordinary Differential Equations I: Nonstiff Problems, vol. 8 of Springer Series in Computational Mathematics, Springer, Berlin, Germany, 1993.

[71] E. Hairer and G. Wanner, Solving Ordinary Differential Equations II. Stiff and Differential-Algebraic Problems, Springer, Berlin, Germany, 2002.

[72] B. Øksendal, Stochastic Differential Equations. An Introduction with Applications, Springer, Amsterdam, The Netherlands, 2005.

[73] M. Bar-Yam, Dynamics of Complex Systems, Westview Press, Boulder, Colo, USA, 1997.

[74] C. S. Bertuglia and F. Vaio, Nonlinearity, Chaos, and Complexity, Oxford University Press, New York, NY, USA, 2005.

[75] Y. Chen and A. Y. T. Leung, Bifurcation and Chaos in Engineering, Springer, London, UK, 1998.

[76] W. L. Cleghorn, Mechanics of Machines, Oxford University Press, New York, NY, USA, 2005.

[77] D. Condurache and V. Martinusi, "Foucault Pendulum-like problems: a tensorial approach," International Journal of NonLinear Mechanics, vol. 43, no. 8, pp. 743-760, 2008.

[78] J. M. Cushing, R. F. Costantino, B. Dennis, R. A. Desharnais, and S. M. Henson, Chaos in Ecology. Experimental Nonlinear Dynamics, Academic Press, Elsevier Science, San Diego, Calif, USA, 2003.

[79] R. L. Devaney, An Introduction To Chaotic Dynamical Systems, Westview Press, Boulder, Colo, USA, 2003.

[80] K. Falconer, Fractal Geometry: Mathematical Foundations and Applications, John Wiley \& Sons, Second edition, 2003.

[81] A. Ghosal, Robotics: Fundamental Concepts and Analysis, Oxford University Press, New Delhi, India, 2006.

[82] D. T. Greenwood, Advanced Dynamics, Cambridge University Press, New York, NY, USA, 2006.
[83] M. de Icaza-Herrera and V. M. Castaño, "Generalized Lagrangian of the parametric foucault pendulum with dissipative forces," Acta Mechanica, vol. 218, no. 1-2, pp. 45-64, 2011.

[84] O. D. Johns, Analytical Mechanics for Relativity and Quantum Mechanics, Oxford University Press, New York, NY, USA, 2005.

[85] J. V. José and E. J. Saletan, Classical Dynamics: A Contemporary Approach, Cambridge University Press, Cambridge, Mass, USA, 1998.

[86] J. Kortelainen and A. Mikkola, "Semantic data model in multibody system simulation," Proceedings of the Institution of Mechanical Engineers, Part K: Journal of Multi-Body Dynamics, vol. 224, no. 4, pp. 341-352, 2010.

[87] J. G. Papastavridis, Analytical Mechanics. A Comprehensive Treatise on the Dynamics of Constrained Systems; for Engineers, Physicists, Oxford University Press, New York, NY, USA, 2001.

[88] M. Pardy, "Bound motion of bodies and particles in the rotating systems," International Journal of Theoretical Physics, vol. 46, no. 4, pp. 848-859, 2007.

[89] A. Preumont, Vibration Control of Active Structures, vol. 179 of Solid Mechanics and its Applications, Kluwer Academic, Dordrecht, The Netherlands, 2011.

[90] W. F. Smith, Waves and Oscillations. A Prelude to Quantum Mechanics, Oxford University Press, New York, NY, USA, 2010.

[91] F. Scheck, Mechanics. From Newton's Laws to Deterministic Chaos, Graduate Texts in Physics, Springer, Heidelberg, Germany, 2010.

[92] A. Smaili and F. Mrad, Applied Mechatronics, Oxford University Press, New York, NY, USA, 2008.

[93] S. H. Strogatz, Nonlinear Dynamics and Chaos: With Applications to Physics, Biology, Chemistry, and Engineering, Westview Press, Boulder, Colo, US, 2000.

[94] B. H. Tongue, Principles of Vibration, Oxford University Press, New York, NY, USA, 2002.

[95] A. Tözeren, Human Body Dynamics: Classical Mechanics and Human Movement, Springer, New York, NY, USA, 2000.

[96] F. E. Udwadia and R. E. Kalaba, Analytical Dynamics: A New Approach, Cambridge University Press, New York, NY, USA, 1996.

[97] J. Uicker, G. Pennock, and J. Shigley, Theory of Machines and Mechanisms, Oxford University Press, New York, NY, USA, 2003.

[98] M. A. Wahab, Dynamics and Vibration: An Introduction, John Wiley \& Sons, The Atrium, UK, 2008.

[99] A. Zanzottera, G. Mingotti, R. Castelli, and M. Dellnitz, "Lowenergy Earth-to-halo transfers in the Earth-Moon scenario with Sun-perturbation," in Nonlinear and Complex Dynamics: Applications in Physical, Biological, and Financial Systems, J. A. T. Machado, D. Baleanu, and A. C. J. Luo, Eds., pp. 39-51, Springer, New York, NY, USA, 2011.

[100] V. F. Zhuravlev and A. G. Petrov, "The lagrange top and the foucault pendulum in observed variables," Doklady Physics, vol. 59, no. 1, pp. 35-39, 2014. 

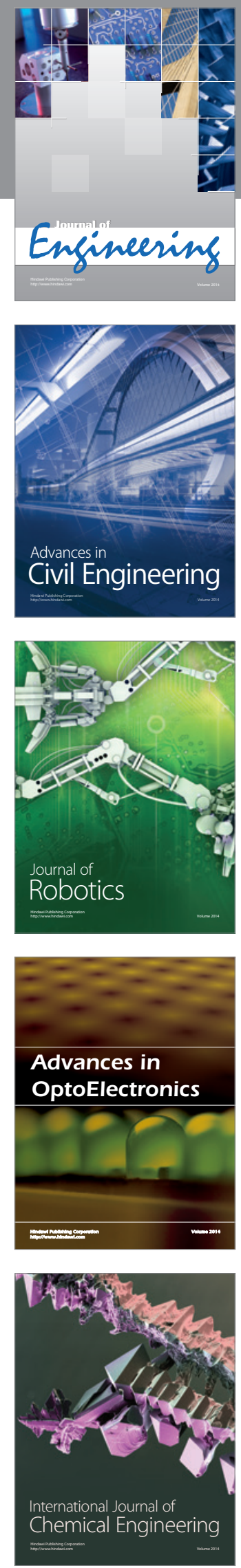

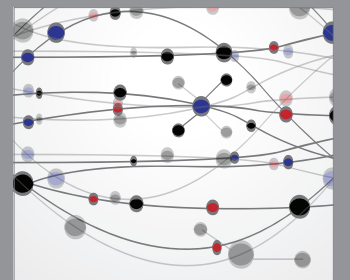

The Scientific World Journal
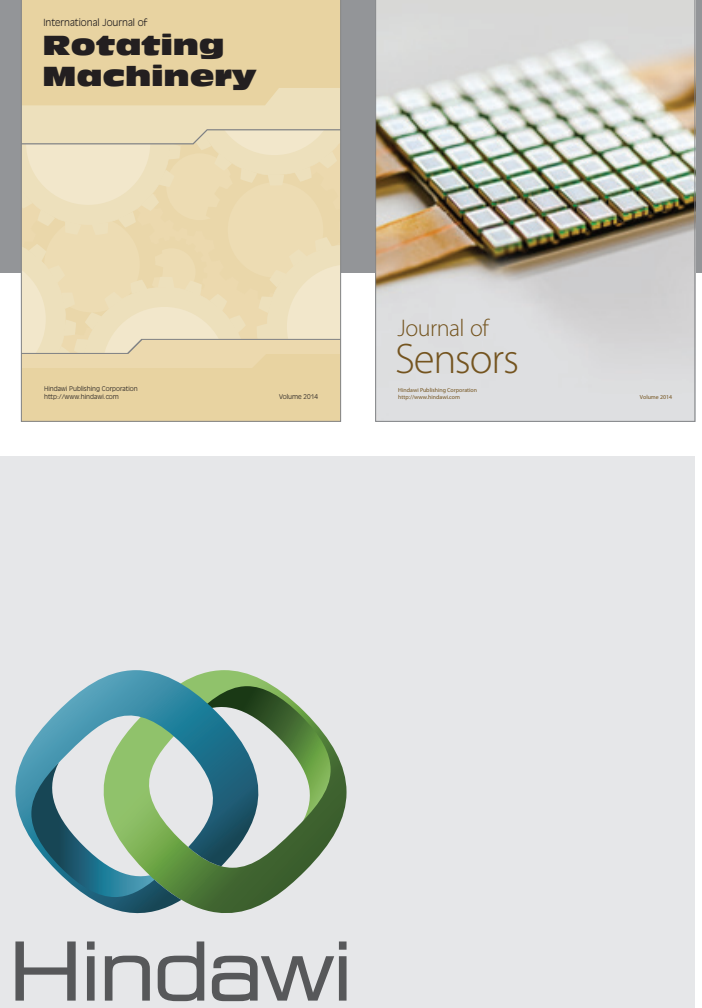

Submit your manuscripts at http://www.hindawi.com
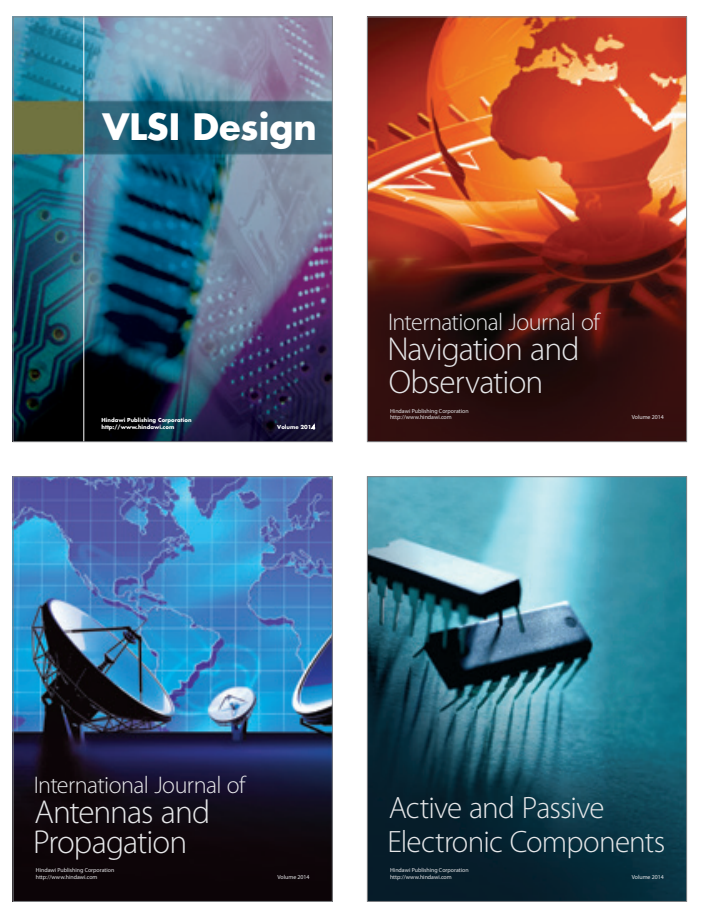
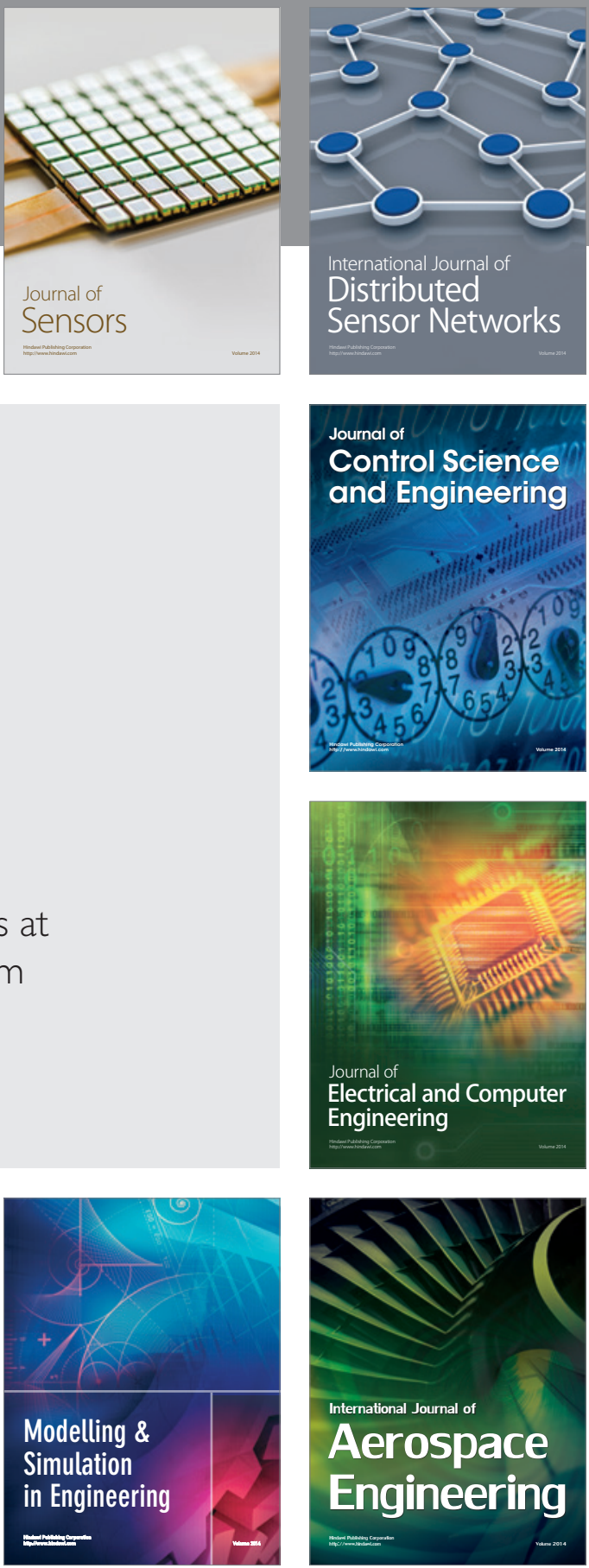

Journal of

Control Science

and Engineering
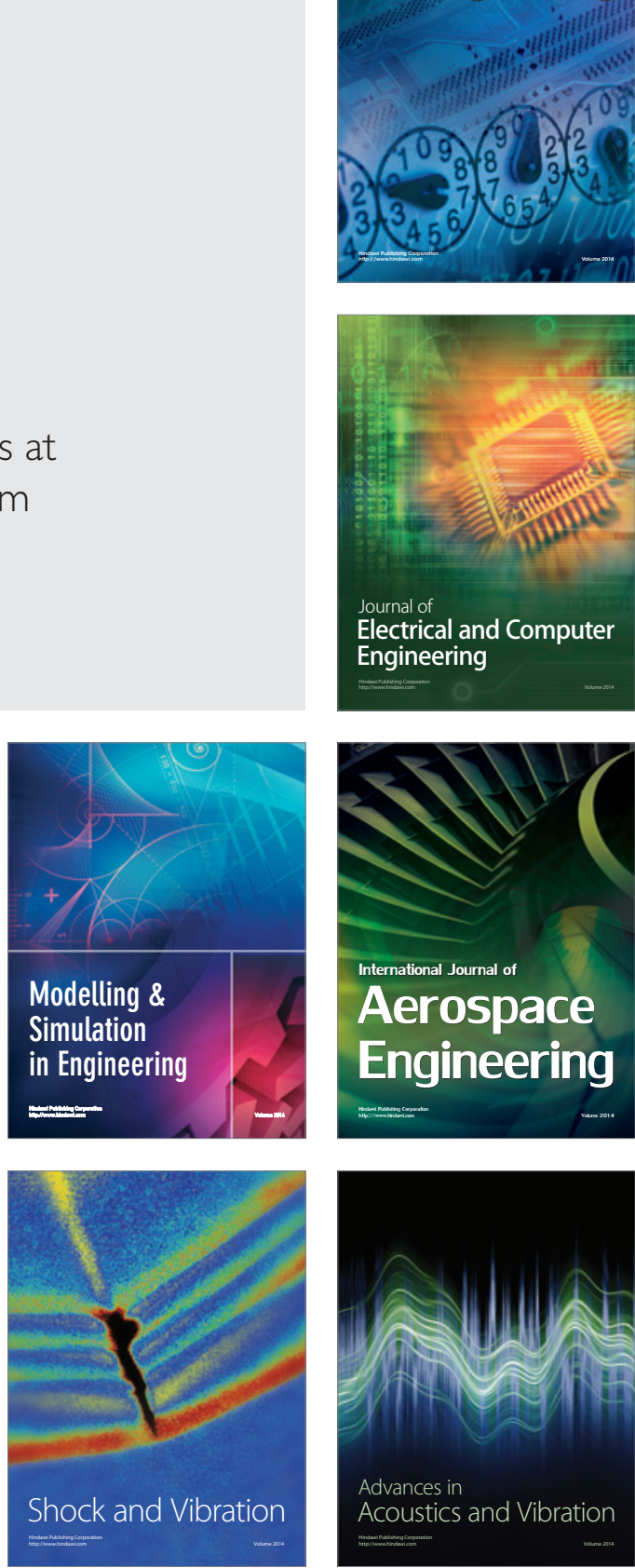